\title{
An Introduction to Supersymmetric Gauge Theories and Matrix Models
}

\author{
Riccardo Argurio, \\ Physique Théorique et Mathématique \\ Université Libre de Bruxelles, C.P. 231, 1050 Bruxelles, Belgium \\ Gabriele Ferretti and Rainer Heise \\ Institute for Theoretical Physics - Göteborg University and \\ Chalmers University of Technology, 412 96 Göteborg, Sweden
}

\begin{abstract}
We give an introduction to the recently established connection between supersymmetric gauge theories and matrix models. We begin by reviewing previous material that is required in order to follow the latest developments. This includes the superfield formulation of gauge theories, holomorphy, the chiral ring, the Konishi anomaly and the large $N$ limit. We then present both the diagrammatic proof of the connection and the one based on the anomaly. Our discussion is entirely field theoretical and self contained.
\end{abstract}




\section{Contents}

$\begin{array}{lll}1 & \text { Introduction } & 1\end{array}$

2 Superspace formulation of gauge theories 3

2.1 Chiral superfields . . . . . . . . . . . . . . . 7

2.2 Vector superfields . . . . . . . . . . . . . . . . . 10

2.3 The complete Lagrangian . . . . . . . . . . . . . . . . . 13

3 Basic Dynamical Facts 15

4 Diagrammatic computation of the glueball superpotential 23

$\begin{array}{lll}5 & \text { The chiral ring } & 38\end{array}$

6 The Konishi anomalv 45

7 Glueball superpotential via the Konishi anomalv 48

\begin{tabular}{ll}
\hline A Superspace notation & 61
\end{tabular}

B One cut solution to the cubic matrix model 62

\begin{tabular}{ll}
\hline C Bibliographical note & 68
\end{tabular}

\section{Introduction}

The scope of this review is to give a pedagogical introduction to some new techniques developed in the context of supersymmetric gauge theories, enabling one to compute systematically non-perturbative quantities in the low energy effective physics. These new developments were initiated by a conjecture put forward by Dijkgraaf and Vafa [1] where it was argued that non-perturbative quantities such as the glueball superpotential can be computed by means of a matrix model.

The main motivation for the study of supersymmetric (SUSY) gauge theories is of course that they are expected to be directly relevant to the next round of high energy experiments, where evidence for the supersymmetric partners of the standard model particles should hopefully be found. To be phenomenologically viable, supersymmetry must of course be broken in some way. While this is the main phenomenological context for the study of supersymmetry, there are further theoretical reasons for studying 
SUSY gauge theories. These theories, even when preserving supersymmetry (to be precise, minimal $\mathcal{N}=1$ SUSY), are believed to share many key dynamical features with QCD such as confinement, dynamical generation of a mass gap in the gauge sector, chiral symmetry breaking and the related non-trivial vacuum structure. All these features are non-perturbative in nature and notoriously difficult to address in real life QCD. With unbroken SUSY there are a number of powerful tools at one's disposal, such as non-renormalization theorems and holomorphy. While important dynamical issues such as confinement remain out of reach and have to be assumed, it is possible to make exact statements concerning the vacuum structure of the theory and the value of the vacuum condensates.

The quantitative investigation of the non-perturbative aspects of SUSY gauge theories began in the early 80's, as reviewed in [2], and received new impetus during the 90's, see [3]. Subsequently, in the second part of the 90 's it was realized that there are a huge number of possibilities to embed gauge theories within string theory, using D-branes and dualities. This led to an enormous amount of work in many directions, one of which 4 culminated in the proposal of [1]. This conjecture, originally motivated in the above string theory context, was subsequently given a purely field theoretical interpretation in various different approaches. In [5] the emphasis was on the relation with the Seiberg-Witten solution of $\mathcal{N}=2$ pure gauge theory [6], broken to $\mathcal{N}=1$ by the presence of a tree level superpotential for the adjoint matter field. In [7] a purely perturbative argument was given, in which the glueball superfield was treated as a classical background field. Finally, in [8] the connection with a matrix model was made using generalized Konishi anomaly relations [9].

In this review we will concentrate on the purely field theoretical $\mathcal{N}=1$ approach to these new techniques, very much in the spirit of [7] and [8]. This choice is partly dictated by the wish to provide a self contained introduction using the least amount of advanced results. Hopefully this will equip the reader with the basic knowledge to address the more advanced current literature on the subject. In this spirit, we have made some effort to discuss earlier results in $\mathcal{N}=1$ gauge theories to put the new developments into context.

The review is organized as follows. In Section 2 we briefly present the superspace formulation of $\mathcal{N}=1$ gauge theory. This is the language in which all the results will be derived. We introduce chiral and vector superfields and show how they combine into the most general gauge theory with matter. Section 3 discusses some basic dynamical properties of these theories such as the perturbative non-renormalization theorem, the holomorphy of 
the Wilsonian superpotential, the linearity principle and the formulation of the low energy physics in terms of the glueball superfield. The review of the latest developments begins in Section 4 where the conjecture of Dijkgraaf and Vafa [1] is presented and derived in a perturbative context. This section describes the computation of [7] by considering the simplest example, namely that of a cubic superpotential for matter in the adjoint representation of $U(N)$. We show explicitly the equivalence between the field theory computation and the matrix model concentrating on a simple diagram. Furthermore, we show that only planar diagrams are relevant to the problem. Section 5 introduces the concept of the chiral ring, which provides a useful tool to analyze the vacuum structure of the theory. To do so, we define chiral operators and derive their basic properties. We then introduce an equivalence relation between chiral operators leading to the concept of the chiral ring and discuss its quantum corrections. Section 6 describes the Konishi anomaly [9] and its generalizations [8]. Finally, in Section 7 we show how one can determine the effective superpotential, using the results of the two previous sections, and how this is related to the matrix model. This last section is closely based on 8 .

Appendix A fixes the notation. Appendix B reviews the one cut solution to the matrix model of [10], needed for the complete solution of the example in Section 4. In Appendix $\mathrm{C}$ we make a humble attempt at reviewing the literature that uses the new techniques discussed here. With this we hope to partly correct for not having touched many interesting new developments in the main text. A second apology is due for omitting many important results in SUSY gauge theories previous to the Dijkgraaf and Vafa conjecture, let alone for not mentioning extended supersymmetry. Fortunately there are many extensive reviews covering these subjects.

\section{Superspace formulation of gauge theories}

The purpose of this section is to familiarize the reader with the techniques of superspace which has the advantage of making SUSY manifest, see e.g. [1] for a list of references to the original papers. We begin by showing what a SUSY Lagrangian looks like in a non manifestly SUSY notation (the so called component notation) and then explain how it can be rewritten in a way so as to make SUSY manifest. The reader familiar with the formalism can skip directly to Section 3 .

The two typical examples of four dimensional SUSY Lagrangians are the so called Wess-Zumino (WZ) model (no gauge interactions, only matter 
fields) and the pure gauge theory (only gauge interaction, no matter fields).

The WZ model can be written as [12]:

$$
\mathcal{L}=\partial_{\mu} \bar{\phi} \partial^{\mu} \phi-i \bar{\psi} \bar{\sigma}^{\mu} \partial_{\mu} \psi+\bar{f} f+\text { interactions }
$$

where $\phi$ is a complex scalar, $\psi$ a Weyl fermion ${ }^{1}$ and $f$ an auxiliary field (a field without a kinetic term) that can be integrated out by its own (algebraic) equation of motion. The interaction terms can also be written explicitly and we will discuss them at length in the following. For the purpose of introducing the SUSY transformations however the kinetic terms (10) are enough.

The pure gauge theory on the other hand has a Lagrangian [13]

$$
\mathcal{L}=\operatorname{tr}\left(-\frac{1}{2} F^{\mu \nu} F_{\mu \nu}-2 i \bar{\lambda} \bar{\sigma}^{\mu} D_{\mu} \lambda+D^{2}\right)
$$

where $F_{\mu \nu}$ stands for the usual field strength, $\lambda$ is a Weyl fermion (the "gluino" or "gaugino") and $D$ is, again, an auxiliary field. All fields in (2) are matrix valued in some Lie algebra and the trace is the trace over the generators: $\operatorname{tr}\left(T^{a} T^{b}\right)=\frac{1}{2} \delta^{a b}$. One could also add a so called "topological term" proportional to $\epsilon_{\mu \nu \rho \sigma} \operatorname{tr} F^{\mu \nu} F^{\rho \sigma}$ or, for $U(1)$ factors in the gauge group, a so called Fayet-Iliopoulos (FI) term proportional to $D$ but otherwise gauge invariance prevents any other type of renormalizable interaction.

The most general four dimensional gauge theory with $\mathcal{N}=1$ SUSY is, loosely speaking, based on a combination of (11) and (2) as we are going to show.

The SUSY transformations can be written explicitly as transformations on the fields. In the context of the WZ model, one can show that

$$
\begin{aligned}
\delta \phi & =\xi \psi \\
\delta \psi & =i \sigma^{\mu} \bar{\xi} \partial_{\mu} \phi+\xi f \\
\delta f & =i \bar{\xi} \bar{\sigma}^{\mu} \partial_{\mu} \psi
\end{aligned}
$$

in terms of one ${ }^{2}$ constant Grassmann (i.e. anticommuting) Weyl spinor $\xi_{\alpha}$ $\left(\bar{\xi}_{\dot{\alpha}}=\left(\xi_{\alpha}\right)^{*}\right)$, changes (10) by at most a total derivative, thus leaving the action $S=\int \mathrm{d}^{4} x \mathcal{L}$ invariant. A similar transformation can be shown to

\footnotetext{
${ }^{1}$ Weyl spinors are ubiquitous in SUSY Lagrangians, and the conventions used are slightly different from the more familiar ones involving Dirac spinors. We review the notation in Appendix A.

${ }^{2}$ Thus the name $\mathcal{N}=1$.
} 
leave (2) invariant, again up to a total spacetime derivative:

$$
\begin{aligned}
\delta A^{\mu} & =\frac{1}{\sqrt{2}}\left(-i \bar{\lambda} \bar{\sigma}^{\mu} \xi+i \bar{\xi} \bar{\sigma}^{\mu} \lambda\right) \\
\delta \lambda & =\frac{1}{\sqrt{2}}\left(\sigma^{\mu \nu} \xi F_{\mu \nu}+i \xi D\right) \\
\delta D & =\frac{1}{\sqrt{2}}\left(-\xi \sigma^{\mu} D_{\mu} \bar{\lambda}-D_{\mu} \lambda \sigma^{\mu} \bar{\xi}\right) .
\end{aligned}
$$

While the invariance of (11) under (3) is straightforward, in checking the invariance of (2) under (4) a more involved four Fermi term arises from the variation of the gauge potential $A_{\mu}$ inside $D_{\mu}$, which can be shown to vanish using Fierz identities. For later convenience, in (3) and (4) we rescaled the SUSY parameter by a factor of $1 / \sqrt{2}$ from that which is commonly used.

The formulation briefly sketched above is manifestly Lorentz and gauge invariant, but lacks manifest supersymmetry invariance. If we had such a manifest SUSY formulation we would not need to go through the explicit computations discussed in the previous paragraph. This task is accomplished by noticing that the SUSY transformations can be written in terms of "translations in superspace" [14. To understand what that means, let us consider an ordinary translation of a scalar field $\phi(x)$. We know that there exist an operator $\mathcal{P}^{\mu}$ such that

$$
\phi(x+a)=e^{-i a \mathcal{P}} \phi(x) e^{i a \mathcal{P}} .
$$

When $a$ is small we can expand both sides. On the one hand:

$\phi(x+a)=\left(1-i a^{\mu} \mathcal{P}_{\mu}+\ldots\right) \phi(x)\left(1+i a^{\mu} \mathcal{P}_{\mu}+\ldots\right)=\phi(x)-i a^{\mu}\left[\mathcal{P}_{\mu}, \phi(x)\right]+\ldots$

on the other hand

$$
\phi(x+a)=\phi(x)+a^{\mu} \partial_{\mu} \phi(x)+\ldots
$$

Thus:

$$
\left[\mathcal{P}_{\mu}, \phi(x)\right]=i \partial_{\mu} \phi(x) \equiv P_{\mu} \phi(x) .
$$

Hence, we have two objects playing the role of "momentum": $\mathcal{P}_{\mu}$ which is the generator of translations and $P_{\mu} \equiv i \partial_{\mu}$ which gives the representation of $\mathcal{P}_{\mu}$ in field space. These two objects are often written using the same symbol.

Supersymmetry is the generalization of this to "superspace", that is space parameterized by the usual coordinates $x^{\mu}$ and new complex Grassmann (anti-commuting) coordinates $\theta^{\alpha}$ and $\bar{\theta}^{\dot{\alpha}}=\left(\theta^{\alpha}\right)^{*}$. A "superfield" is a 
function in superspace: $Y(x, \theta, \bar{\theta})$. Since $\left(\theta_{1}\right)^{2}=\left(\theta_{2}\right)^{2}=0$ and similarly for $\bar{\theta}$ due to anticommutativity, the Taylor expansion of $Y$ in $\theta$ and $\bar{\theta}$ terminates:

$$
\begin{aligned}
Y(x, \theta, \bar{\theta})= & \phi(x)+\theta \eta(x)+\bar{\theta} \bar{\chi}(x)+\theta^{2} m(x)+\bar{\theta}^{2} n(x)+\theta \sigma^{\mu} \bar{\theta} A_{\mu}(x)+ \\
& \theta^{2} \bar{\theta} \bar{\lambda}(x)+\bar{\theta}^{2} \theta \psi(x)+\theta^{2} \bar{\theta}^{2} d(x) .
\end{aligned}
$$

We have not indicated it explicitly but $Y$ could carry some spin indices that would reflect in the component structure. The above formula refers to a Lorentz scalar for simplicity.

In analogy with usual translations, we now define translations in superspace by a quantity $(a, \xi, \bar{\xi})$ as:

$$
\begin{aligned}
\theta_{\alpha} & \rightarrow \theta_{\alpha}+\xi_{\alpha} \\
\bar{\theta}_{\dot{\alpha}} & \rightarrow \bar{\theta}_{\dot{\alpha}}+\bar{\xi}_{\dot{\alpha}} \\
x^{\mu} & \rightarrow x^{\mu}+a^{\mu}+\frac{i}{2} \theta \sigma^{\mu} \bar{\xi}-\frac{i}{2} \xi \sigma^{\mu} \bar{\theta}
\end{aligned}
$$

where one should note the crucial addition of the fermionic bilinears to the shift of $x^{\mu}$. They are of course needed so that the composition of two fermionic translations yields a translation in $x^{\mu}$.

The operators generating such transformation on the superfields are $\mathcal{P}$, $\mathcal{Q}$ and $\overline{\mathcal{Q}}$ obeying

$$
\begin{aligned}
& Y\left(x^{\mu}+a^{\mu}+\frac{i}{2} \theta \sigma^{\mu} \bar{\xi}-\frac{i}{2} \xi \sigma^{\mu} \bar{\theta}, \theta_{\alpha}+\xi_{\alpha}, \bar{\theta}_{\dot{\alpha}}+\bar{\xi}_{\dot{\alpha}}\right)= \\
& e^{-i a \mathcal{P}+\xi \mathcal{Q}-\bar{\xi} \overline{\mathcal{Q}}} Y\left(x^{\mu}, \theta_{\alpha}, \bar{\theta}_{\dot{\alpha}}\right) e^{i a \mathcal{P}-\xi \mathcal{Q}+\bar{\xi} \overline{\mathcal{Q}}} .
\end{aligned}
$$

Not surprisingly, the operators $\mathcal{Q}$ and $\overline{\mathcal{Q}}$ are also represented by differential operators acting on superfields, as one can check:

$$
\left[\mathcal{Q}_{\alpha}, Y\right]=Q_{\alpha} Y, \quad\left[\overline{\mathcal{Q}}_{\dot{\alpha}}, Y\right]=\bar{Q}_{\dot{\alpha}} Y
$$

where on the left hand side we have a commutator or anticommutator depending on the spin of $Y$. Explicitly:

$$
Q_{\alpha}=\partial_{\alpha}-\frac{i}{2} \sigma_{\alpha \dot{\alpha}}^{\mu} \bar{\theta}^{\dot{\alpha}} \partial_{\mu}, \quad \bar{Q}_{\dot{\alpha}}=\bar{\partial}_{\dot{\alpha}}-\frac{i}{2} \theta^{\alpha} \sigma_{\alpha \dot{\alpha}}^{\mu} \partial_{\mu}
$$

One can show that $P, Q$ and $\bar{Q}$ obey the SUSY algebra

$$
\left\{Q_{\alpha}, \bar{Q}_{\dot{\alpha}}\right\}=-\sigma_{\alpha \dot{\alpha}}^{\mu} P_{\mu} .
$$


Having seen that SUSY is a translation in superspace, any integral over superspace of a superfield $Y$ :

$$
\int \mathrm{d}^{4} x \mathrm{~d}^{2} \theta \mathrm{d}^{2} \bar{\theta} Y
$$

will be manifestly supersymmetric because the integration measure is translational invariant by construction (see Appendix A). Of course, $Y$ has to be a scalar superfield and can arise as the composition of other superfields.

In the case of translations we were done at this point. Here we have to perform an extra step because the generic superfield $Y$ above forms a reducible representation of the SUSY algebra (14), that is, it is possible to restrict the form of $Y$ to contain only a subset of the original fields that are still mapped into each other by SUSY. It should be obvious that $Y$ is "too big" because we would like, for instance, to formulate the WZ model (11) which does not have any vector fields or the pure gauge theory (2) which does not have any scalars. The two ways of reducing the field content in $Y$, that are going to be used are: (i) The chiral (or antichiral) projection and (ii) The real projection.

\subsection{Chiral superfields}

A chiral superfield can be constructed by noticing that there exist two differential operators:

$$
D_{\alpha}=\partial_{\alpha}+\frac{i}{2} \sigma_{\alpha \dot{\alpha}}^{\mu} \bar{\theta}^{\dot{\alpha}} \partial_{\mu}, \quad \bar{D}_{\dot{\alpha}}=\bar{\partial}_{\dot{\alpha}}+\frac{i}{2} \theta^{\alpha} \sigma_{\alpha \dot{\alpha}}^{\mu} \partial_{\mu}
$$

that anticommute with all the supercharges (13). This means that if $Y$ is a superfield, so are $D_{\alpha} Y$ and $\bar{D}_{\dot{\alpha}} Y$ and it is consistent with SUSY to set one of them to zero. (Setting both would result in requiring $Y=$ const.) We call chiral superfield a superfield $\Phi$ obeying $\bar{D}_{\dot{\alpha}} \Phi=0$. (Analogously, $D_{\alpha} \bar{\Phi}=0$ defines an antichiral superfield.)

The solution to $\bar{D}_{\dot{\alpha}} \Phi=0$ can be obtained by observing that the (complex) combination $y^{\mu}=x^{\mu}+\frac{i}{2} \theta \sigma^{\mu} \bar{\theta}$ obeys $\bar{D}_{\dot{\alpha}} y^{\mu}=0$, and so does trivially $\theta_{\alpha}$. Thus

$$
\Phi=\phi(y)+\theta \psi(y)+\theta^{2} f(y)
$$

is the general expression for a chiral superfield. Expanding (17) around $x$ yields

$\Phi=\phi(x)+\frac{i}{2} \theta \sigma^{\mu} \bar{\theta} \partial_{\mu} \phi(x)-\frac{1}{4} \theta^{2} \bar{\theta}^{2} \square \phi(x)+\theta \psi(x)-\frac{i}{2} \theta^{2} \partial_{\mu} \psi(x) \sigma^{\mu} \bar{\theta}+\theta^{2} f(x)$ 
Let us begin by considering the action of $\mathcal{Q}$ on $\Phi$. Recalling that SUSY can be written as a translation in superspace, we have, on the one hand ${ }^{3}$ :

$$
\delta_{\xi} \Phi=\xi Q \Phi=\xi \psi(y)+\xi \theta f(y)
$$

on the other hand:

$$
\delta_{\xi} \Phi=[\xi \mathcal{Q}, \Phi]=[\xi \mathcal{Q}, \phi]+\theta^{\alpha}\left[\xi \mathcal{Q}, \psi_{\alpha}\right]+\theta^{2}[\xi \mathcal{Q}, f]
$$

Thus, the transformation properties (3) are reproduced, now written in terms of the SUSY generators

$$
\begin{aligned}
{\left[\mathcal{Q}_{\alpha}, \phi\right] } & =\psi_{\alpha} \\
\left\{\mathcal{Q}_{\alpha}, \psi_{\beta}\right\} & =-\epsilon_{\alpha \beta} f \\
{\left[\mathcal{Q}_{\alpha}, f\right] } & =0 .
\end{aligned}
$$

Similarly, one can check that

$$
\begin{aligned}
{\left[\overline{\mathcal{Q}}_{\dot{\alpha}}, \phi\right] } & =0 \\
\left\{\overline{\mathcal{Q}}_{\dot{\alpha}}, \psi_{\beta}\right\} & =-i \sigma_{\beta \dot{\alpha}}^{\mu} \partial_{\mu} \phi \\
{\left[\overline{\mathcal{Q}}_{\dot{\alpha}}, f\right] } & =i \sigma_{\alpha \dot{\alpha}}^{\mu} \partial_{\mu} \psi^{\alpha} .
\end{aligned}
$$

In order to write a SUSY action we recall that the superfield $Y$ in (15) can be the composition of other superfields. For instance, given a chiral superfield $\Phi$ one can write

$$
\int \mathrm{d}^{4} x \mathrm{~d}^{2} \theta \mathrm{d}^{2} \bar{\theta} \bar{\Phi} \Phi
$$

which has been chosen as an example because by expanding $\Phi$ and $\bar{\Phi}$ and integrating over $\theta$ and $\bar{\theta}$ we obtain precisely the kinetic terms in the WZ model (1).

It is often stated that there is an exception to (15) when the integrand happens to be a chiral superfield $\Sigma$ (or a product of such). In this case, the integral over the whole superspace vanishes, however, the integral over chiral superspace

$$
\int \mathrm{d}^{4} x \mathrm{~d}^{2} \theta \Sigma
$$

is still manifestly SUSY (translation invariant) as can readily be seen in the formalism where $x^{\mu}$ is replaced by $y^{\mu}$ so that $\bar{\theta}$ never appears. Equivalently,

\footnotetext{
${ }^{3}$ The SUSY transformation of the coordinate $y^{\mu}$ is independent of $\xi$. We take formally $\bar{\xi}=0$ since we are only interested in $Q$ and not $\bar{Q}$.
} 
it can be noticed from eqs. (21) and (22) that the highest component $f$ of a chiral superfield (which is singled out by the $\theta^{2}$ integral) transforms as a total spacetime derivative.

However, it should be appreciated that (24) is actually more general than (15) in the sense that any integral of type (15) can be written as (24). Remember that integrals over a Grassmann variable are equivalent to derivatives and that total spacetime derivatives $\partial_{\mu}$ do not contribute to the bosonic integral ${ }^{4}$ so that one can write

$$
\int \mathrm{d}^{4} x \mathrm{~d}^{2} \theta \mathrm{d}^{2} \bar{\theta} Y=\int \mathrm{d}^{4} x \mathrm{~d}^{2} \theta \bar{D}^{2} Y .
$$

The superfield $\bar{D}^{2} Y$ is manifestly chiral, regardless of the form of $Y$ because three $\bar{D}_{\dot{\alpha}}$ always give zero since they anticommute and there are only two possible values $\dot{\alpha}$ can take. We call such terms "chirally exact". On the other hand, not all terms of the form (24) can be written as (15) in terms of a local integral. For instance, the quantity

$$
\int \mathrm{d}^{4} x \mathrm{~d}^{2} \theta \Phi^{n}
$$

for some chiral superfield $\Phi$, cannot be expressed as (15) because we do not have any derivative that can be reconverted into a $\mathrm{d}^{2} \bar{\theta}$. We call terms such as (26) that cannot be written as local integrals over the whole superspace "F-terms" and all the others, including (25), "D-terms".

The above expression (23) is so compact that it may be a bit confusing at first - for instance, if one tried to obtain the equations of motion by varying $\bar{\Phi}$ one would get the nonsensical result $\Phi=0$. The error, of course, is that the field $\Phi$ obeys $\bar{D}_{\dot{\alpha}} \Phi=0$ but the action (23) doesn't know that. An easy way to enforce this is to use the above tricks and write

$$
\int \mathrm{d}^{4} x \mathrm{~d}^{2} \theta \mathrm{d}^{2} \bar{\theta} \bar{\Phi} \Phi=\int \mathrm{d}^{4} x \mathrm{~d}^{2} \bar{\theta} \bar{\Phi}\left(D^{2} \Phi\right)
$$

Now the fields are unconstrained in (anti)-chiral superspace and varying with respect to $\bar{\Phi}$ yields $D^{2} \Phi=0$ which implies the free equations of motion for $\phi, \psi$ and $f$.

Interactions can be introduced in the same way. Since the simplest nonvanishing integral over all of superspace (23) already involves derivatives, we must turn to terms like (26). In fact any holomorphic function $W(\Phi)$ (i.e.

\footnotetext{
${ }^{4}$ We need not worry about boundary terms at this stage.
} 
such that $\partial W / \partial \bar{\Phi}=0)$ to be called the superpotential, will be acceptable in the sense that if $\Phi$ is chiral, so is $W(\Phi)$ :

$$
\bar{D}_{\dot{\alpha}} W(\Phi)=\frac{\partial W(\Phi)}{\partial \Phi} \bar{D}_{\dot{\alpha}} \Phi+\frac{\partial W(\Phi)}{\partial \bar{\Phi}} \bar{D}_{\dot{\alpha}} \bar{\Phi}=0 .
$$

Thus

$$
\int \mathrm{d}^{4} x \mathrm{~d}^{2} \theta W(\Phi)+\int \mathrm{d}^{4} x \mathrm{~d}^{2} \bar{\theta} \bar{W}(\bar{\Phi})
$$

gives a supersymmetric interaction term that can be added to the kinetic terms in (23). If renormalizability is an issue (and it will not be in many following cases since we will be dealing with effective theories) we must restrict $W$ to be at most a cubic polynomial.

\section{$2.2 \quad$ Vector superfields}

To introduce gauge interactions we need a second type of superfield - the vector superfield. This is just the original $Y$ (conventionally called $V$ in this case and Lie algebra valued) on which we impose the reality condition

$$
V=V^{\dagger}
$$

This condition is trivially supersymmetrically covariant because if $V$ is the most arbitrary superfield, so is $V^{\dagger}$ and equating the two is a superfield equation. In components, displaying the Lie algebra index:

$$
\begin{aligned}
V^{a}= & C^{a}(x)+i \theta \chi^{a}(x)-i \bar{\theta} \bar{\chi}^{a}(x)+i \theta^{2} m^{a}(x)-i \bar{\theta}^{2} \bar{m}^{a}(x)-\theta \sigma^{\mu} \bar{\theta} A_{\mu}^{a}(x) \\
& +\sqrt{2} i \theta^{2} \bar{\theta}\left(\bar{\lambda}^{a}(x)+\frac{i}{2 \sqrt{2}} \bar{\sigma}^{\mu} \partial_{\mu} \chi^{a}(x)\right)-\sqrt{2} i \bar{\theta}^{2} \theta\left(\lambda^{a}(x)\right. \\
& \left.+\frac{i}{2 \sqrt{2}} \sigma^{\mu} \partial_{\mu} \bar{\chi}^{a}(x)\right)+\theta^{2} \bar{\theta}^{2}\left(D^{a}(x)-\frac{1}{4} \square C^{a}(x)\right)
\end{aligned}
$$

where $C^{a}, A_{\mu}^{a}$ and $D^{a}$ are real and the shifts in $\lambda^{a}$ and $D^{a}$ by derivatives of $\chi^{a}$ and $C^{a}$ are for later convenience. If $T^{a}$ are the generators of the Lie algebra $\left(\left[T^{a}, T^{b}\right]=i f^{a b c} T^{c}\right)$, one can write a matrix valued object $V=V^{a} T^{a}$ obeying $V=V^{\dagger}$ because $V^{a}$ are real and $T^{a}$ hermitian.

Let us now see the relation between the vector superfield and gauge invariance. Consider matter described by a chiral superfield $\Phi$ transforming in some representation (reducible or irreducible) of the Lie algebra. The usual gauge transformation

$$
\Phi \rightarrow \Phi^{\prime}=e^{-i \Lambda} \Phi
$$


keeps this form but now $\Lambda \equiv \Lambda^{a} T^{a}$ must also be promoted to a chiral superfield because otherwise $\Phi^{\prime}$ would no longer be chiral. However there is a problem with the kinetic term because, being $\Lambda$ complex

$$
\bar{\Phi} \Phi \rightarrow \bar{\Phi} e^{i \bar{\Lambda}} e^{-i \Lambda} \Phi \neq \bar{\Phi} \Phi .
$$

Thus, we must compensate by inserting $e^{V}$ between $\bar{\Phi}$ and $\Phi$ and postulate the transformation law:

$$
e^{V} \rightarrow e^{V^{\prime}}=e^{-i \bar{\Lambda}} e^{V} e^{i \Lambda}
$$

so that $\bar{\Phi} e^{V} \Phi$ is gauge invariant.

At first sight, it is not obvious that (34) implies an infinitesimal gauge transformation like

$$
\delta A_{\mu}=\partial_{\mu} \alpha+i\left[A_{\mu}, \alpha\right]
$$

but, in fact, (35) is included in the transformation (34) of the components of $V$ as one should check at least for an Abelian group, where (34) simplifies to $V \rightarrow V^{\prime}=V-i \bar{\Lambda}+i \Lambda$.

Using (34) it is possible to show that one can gauge away many of the fields in the original definition of $V$ and go to the so called Wess-Zumino gauge where

$$
V^{a}=-\theta \sigma^{\mu} \bar{\theta} A_{\mu}^{a}(x)+\sqrt{2} i \theta^{2} \bar{\theta} \bar{\lambda}^{a}(x)-\sqrt{2} i \bar{\theta}^{2} \theta \lambda^{a}(x)+\theta^{2} \bar{\theta}^{2} D^{a}(x) .
$$

In this form, $V$ is no longer a superfield - this is not a SUSY gauge. Indeed acting with $Q$ or $\bar{Q}$ would regenerate the terms that have been set to zero in the WZ gauge. However, these new terms can be once again removed by a new (field dependent) gauge transformation. In other words, in this gauge the SUSY algebra closes up to a gauge transformation.

As for the superpotential, no modification is required. As long as $W$ is holomorphic and gauge invariant in the usual sense, the gauge parameter can be promoted to a full chiral superfield because its complex conjugate is never used. This is often stated by saying that the F-terms are invariant under the complexified gauge group.

We now turn to finding the kinetic term for $V$, i.e. the manifestly SUSY way of writing (2). From the transformation law (34) and the fact that $A_{\mu}$ appears explicitly in $V$ it is clear that we must act with some derivative to construct an object to be identified with the supersymmetric field strength.

It turns out that the right expression is

$$
W_{\alpha}=\bar{D}^{2}\left(e^{-V}\left(D_{\alpha} e^{V}\right)\right)
$$


where we have defined the manifestly chiral superfield $W_{\alpha}{ }^{5}$. The antichiral superfield $\bar{W}_{\dot{\alpha}}$ has a similar definition, obtained from $\bar{W}_{\dot{\alpha}}=\left(W_{\alpha}\right)^{*}$.

It is a nice exercise to check that, under a gauge transformation (34), $W_{\alpha}$ transforms as

$$
W_{\alpha} \rightarrow W_{\alpha}^{\prime}=e^{-i \Lambda} W_{\alpha} e^{i \Lambda}
$$

Note the difference between (38) and (34) - in (38) there is no $\bar{\Lambda}$ appearing and so it is possible to make a gauge invariant combination e.g. by taking traces. In checking (38) one has to notice that, first (34) implies $e^{-V} \rightarrow$ $e^{-i \Lambda} e^{-V} e^{i \bar{\Lambda}}$, second that the $\bar{\Lambda}$ terms commute through $D_{\alpha}$ and cancel and, finally, that the spurious term $\bar{D}^{2} D_{\alpha}\left(e^{i \Lambda}\right)$ vanishes.

$W_{\alpha}$ can be easily computed in the WZ gauge to give

$$
W_{\alpha}=-\sqrt{2} i \lambda_{\alpha}(y)+\theta_{\alpha} D(y)-i \sigma_{\alpha}^{\mu \nu \beta} \theta_{\beta} F_{\mu \nu}(y)+\sqrt{2} \theta^{2} \sigma_{\alpha \dot{\beta}}^{\mu} D_{\mu} \bar{\lambda}^{\dot{\beta}}(y),
$$

where, as usual, expanding in $y$ would introduce additional spacetime derivatives. Since $W_{\alpha}$ starts off with the gaugino, it is often called the gaugino (or gluino) superfield.

The pure gauge theory action (2) can be written in a manifestly SUSY way using the field $W_{\alpha}$ :

$$
\int \mathrm{d}^{4} x \mathrm{~d}^{2} \theta \operatorname{tr} W^{\alpha} W_{\alpha}+\int \mathrm{d}^{4} x \mathrm{~d}^{2} \bar{\theta} \operatorname{tr} \bar{W}_{\dot{\alpha}} \bar{W}^{\dot{\alpha}}
$$

For later purpose, it is convenient to reformulate expression (37) for the field strength $W_{\alpha}$ in terms of (anti)commutators of some new "covariant" derivatives. Define

$$
\bar{\nabla}_{\dot{\alpha}} \equiv \bar{D}_{\dot{\alpha}} \quad \text { and } \quad \nabla_{\alpha} \equiv e^{-V} D_{\alpha} e^{V}
$$

the second equation being intended as an operator, that is $D_{\alpha}$ acts on $e^{V}$ and whatever follows it. Given the two new derivatives, one can define "covariantly chiral/antichiral" fields $\Phi$ (as before) and $\tilde{\Phi}=\bar{\Phi} e^{V}$ obeying $\bar{\nabla}_{\dot{\alpha}} \Phi=0$ and $\nabla_{\alpha} \tilde{\Phi}=0$ (this last expression to be interpreted as a right action.). Evaluating the anticommutator yields:

$$
\left\{\nabla_{\alpha}, \bar{\nabla}_{\dot{\alpha}}\right\}=i \sigma_{\alpha \dot{\alpha}}^{\mu} \partial_{\mu}+\bar{D}_{\dot{\alpha}}\left(e^{-V}\left(D_{\alpha} e^{V}\right)\right) \equiv i \nabla_{\alpha \dot{\alpha}}
$$

The last equality defines the new object $\nabla_{\alpha \dot{\alpha}}$, whose lowest component is the usual covariant derivative. Taking one more commutator we obtain:

$$
W_{\alpha}=\frac{i}{2}\left[\bar{\nabla}^{\dot{\alpha}}, \nabla_{\alpha \dot{\alpha}}\right] .
$$

\footnotetext{
${ }^{5}$ Not to be confused with the superpotential, also conventionally denoted with a $W$ but without any spinor indices.
} 


\subsection{The complete Lagrangian}

Let us summarize all the results thus far by writing the most general $\mathcal{N}=$ 1 gauge theory for a simple gauge group $G$. It is convenient to combine the usual Yang-Mills coupling $g$ and the vacuum angle $\Theta$ into a complex parameter

$$
\tau=\frac{4 \pi}{g^{2}}-i \frac{\Theta}{2 \pi}
$$

Let $\Phi$ be the matter chiral superfield transforming in a (generically reducible) representation of $G$. The complete Lagrangian takes the form

$$
\begin{aligned}
S= & \frac{\tau}{16 \pi} \int \mathrm{d}^{4} x \mathrm{~d}^{2} \theta \operatorname{tr} W^{\alpha} W_{\alpha}+\frac{\bar{\tau}}{16 \pi} \int \mathrm{d}^{4} x \mathrm{~d}^{2} \bar{\theta} \operatorname{tr} \bar{W}_{\dot{\alpha}} \bar{W}^{\dot{\alpha}}+ \\
& \int \mathrm{d}^{4} x \mathrm{~d}^{2} \theta \mathrm{d}^{2} \bar{\theta} \bar{\Phi} e^{V} \Phi+\int \mathrm{d}^{4} x \mathrm{~d}^{2} \theta W(\Phi)+\int \mathrm{d}^{4} x \mathrm{~d}^{2} \bar{\theta} \bar{W}(\bar{\Phi})
\end{aligned}
$$

The superpotential $W(\Phi)$ is invariant under $G$ and all the coupling constants inside it are complex. In fact, in string theory it is quite natural to think of these couplings as the vacuum expectation values of some background chiral superfields as we will discuss later.

It is instructive to write (45) in components in the WZ gauge. The gauge kinetic Lagrangian (first line in eq. (45)) becomes

$$
\frac{1}{g^{2}} \operatorname{tr}\left(-\frac{1}{2} F^{\mu \nu} F_{\mu \nu}-2 i \bar{\lambda} \bar{\sigma}^{\mu} D_{\mu} \lambda+D^{2}\right)+\frac{\Theta}{32 \pi^{2}} \epsilon^{\mu \nu \rho \lambda} \operatorname{tr} F_{\mu \nu} F_{\rho \lambda} .
$$

The matter "kinetic" Lagrangian (first term in the second line of (45) ) reads

$$
D_{\mu} \bar{\phi} D^{\mu} \phi-i \bar{\psi} \bar{\sigma}^{\mu} D_{\mu} \psi+\bar{f} f+\sqrt{2} i(\bar{\phi} \lambda \psi-\bar{\psi} \bar{\lambda} \phi)+\bar{\phi} D \phi
$$

where the indices ${ }^{6}$ are contracted in the only possible way, i.e. $\bar{\phi} \lambda \psi \equiv$ $\bar{\phi}^{i} \lambda^{\alpha a} T_{i}^{a j} \psi_{\alpha j}$. Lastly, the contribution from the superpotential reads

$$
-\frac{1}{2} \frac{\partial^{2} W}{\partial \phi_{i} \partial \phi_{j}} \psi_{i} \psi_{j}+\frac{\partial W}{\partial \phi_{i}} f_{i}+c . c .
$$

Let us collect all terms contributing to the bosonic potential

$$
\mathcal{V}=-\frac{1}{2 g^{2}} D^{a 2}-\bar{\phi} T^{a} \phi D^{a}-\bar{f}^{i} f_{i}-\frac{\partial W}{\partial \phi_{i}} f_{i}-\frac{\partial \bar{W}}{\partial \bar{\phi}^{i}} \bar{f}^{i} .
$$

\footnotetext{
${ }^{6}$ The index $a=1 \ldots \operatorname{dim}(G)$ runs over the adjoint representation of $G$. The indices $i, j=1 \ldots \operatorname{dim}(R)$ label the representation $R$ of $\Phi$ and its conjugate representation $\bar{R}$.
} 
The auxiliary fields $D$ and $f$ appear only in these terms and we can solve for them as

$$
D^{a}=-g^{2} \bar{\phi} T^{a} \phi, \quad f_{i}=-\frac{\partial \bar{W}}{\partial \bar{\phi}^{i}}, \quad \bar{f}^{i}=-\frac{\partial W}{\partial \phi_{i}} .
$$

Substituting the solution (50) in the potential (49) gives

$$
\begin{aligned}
\mathcal{V} & =\frac{g^{2}}{2}\left(\bar{\phi} T^{a} \phi\right)^{2}+\frac{\partial \bar{W}}{\partial \bar{\phi}^{i}} \frac{\partial W}{\partial \phi_{i}} \\
& =\frac{1}{2 g^{2}} D^{a 2}+\bar{f}^{i} f_{i},
\end{aligned}
$$

the last line to be interpreted as evaluated at the solution.

The potential $\mathcal{V}$ is obviously non-negative, and the classical SUSY vacua are described by $\mathcal{V}=0$, i.e. by the simultaneous vanishing of the $D$ and $f$ terms, referred to as D-flatness and F-flatness conditions.

The space of solutions to the D-flatness conditions $\bar{\phi} T^{a} \phi=0$ up to gauge transformations is an important object known as the classical moduli space of the theory. An extremely useful mathematical fact ${ }^{7}$ is that such space can always be parameterized in terms of a set of independent holomorphic gauge invariants $X_{r}(\phi)$. One of the most common examples is a field $\Phi$ in the adjoint representation of $S U(N)$. In this case the D-flatness condition is $\bar{\phi} T^{a} \phi=i f^{a b c} \bar{\phi}^{b} \phi^{c}=0$, that is, $\left[\phi^{\dagger}, \phi\right]=0$ as a matrix equation. The classical moduli space is parameterized by $X_{k}=\operatorname{tr} \phi^{k}$ for $k=2, \ldots N$.

As for the F-flatness condition, it implies that when a superpotential is present classical SUSY vacua are found at its extrema. Thus, in the presence of a superpotential, some of the "moduli" $X_{r}$ defined above may be subjected to further restrictions.

So far our discussion has been in terms of the classical theory and one must ask how this picture is modified in the quantum theory. We will see that, in general, the superpotential will be modified by (non-perturbative) quantum corrections but the property of the superpotential of identifying the SUSY vacua carries over to the low energy effective theory as long as one does not encounter any singularity, typically due to the presence of extra massless fields. In this review we will discuss at length how the superpotential is modified by quantum corrections but unfortunately we will not have time to discuss the many applications to the search for SUSY vacua.

\footnotetext{
${ }^{7}$ For a careful explanation accessible to physicists and a list of earlier references see [15.
} 


\section{Basic Dynamical Facts}

Having seen the kinematic (classical) structure of SUSY gauge theories we now review some basic facts about their (quantum) dynamics. The aim of this section is to present enough information to be able to put the latest developments into context, without any ambition of being exhaustive.

It is a familiar fact that in a quantum theory the couplings appearing in the physical quantities (observables) are actually running parameters depending on the renormalization scale $\mu$ and that they appear in the observables in such a way that the total $\mu$ dependence cancels. All of this is still true in SUSY gauge theories but SUSY implies some simplifications in the running of the coupling constants.

The simplest (and very important) example of this fact is the perturbative non-renormalization of the superpotential. This can already be seen in the WZ model, defined by the bare action ${ }^{8}$ :

$$
S=\int \mathrm{d}^{4} x \mathrm{~d}^{2} \theta \mathrm{d}^{2} \bar{\theta} \bar{\Phi}_{0} \Phi_{0}+\int \mathrm{d}^{4} x \mathrm{~d}^{2} \theta \frac{m_{0}}{2} \Phi_{0}^{2}+\frac{\lambda_{0}}{3} \Phi_{0}^{3}+\text { c.c. }
$$

Loop computations and renormalization can be done in a variety of equivalent ways - we use the so called "renormalized perturbation theory" and write action (52) in terms of the renormalized quantities:

$$
\begin{aligned}
S= & \int \mathrm{d}^{4} x \mathrm{~d}^{2} \theta \mathrm{d}^{2} \bar{\theta} Z_{\Phi} \bar{\Phi} \Phi+\int \mathrm{d}^{4} x \mathrm{~d}^{2} \theta \quad Z_{m} \frac{m}{2} \Phi^{2}+Z_{\lambda} \frac{\lambda}{3} \Phi^{3}+\text { c.c. }= \\
= & \int \mathrm{d}^{4} x \mathrm{~d}^{2} \theta \mathrm{d}^{2} \bar{\theta} \bar{\Phi} \Phi+\int \mathrm{d}^{4} x \mathrm{~d}^{2} \theta \frac{m}{2} \Phi^{2}+\frac{\lambda}{3} \Phi^{3}+\text { c.c. }+ \\
& \int \mathrm{d}^{4} x \mathrm{~d}^{2} \theta \mathrm{d}^{2} \bar{\theta} \delta_{\Phi} \bar{\Phi} \Phi+\int \mathrm{d}^{4} x \mathrm{~d}^{2} \theta \frac{\delta_{m}}{2} \Phi^{2}+\frac{\delta_{\lambda}}{3} \Phi^{3}+\text { c.c. }
\end{aligned}
$$

It is possible to develop Feynman rules directly in superspace without having to expand into component fields. Without presenting the details that are explained very clearly in the literature, the main point [16] is that the most general term that can be generated in loop diagrams has only one Grassmann integral over all four $\theta$ :

$$
\int \mathrm{d}^{4} x_{1} \ldots \mathrm{d}^{4} x_{n} \mathrm{~d}^{2} \theta \mathrm{d}^{2} \bar{\theta} G\left(x_{1} \ldots x_{n}\right) F_{1}\left(x_{1}, \theta, \bar{\theta}\right) \ldots F_{n}\left(x_{n}, \theta, \bar{\theta}\right),
$$

where the $F_{i}$ stand for products of superfields and their derivatives. We notice in passing that the expression would be valid if we also had the gauge

\footnotetext{
${ }^{8}$ We consider a cubic superpotential to ensure that the full theory is renormalizable in the ordinary sense.
} 
superfield $V$ with the possibility of the $F_{i}$ being equal to $V, D_{\alpha} V$ etc. in (54).

We know that the theory is renormalizable because it is so in components. Thus the only possible primitively ultraviolet (UV) divergent graphs are those with an external field structure already present in the bare Lagrangian. Suppose now that there were a UV divergent graph with only two (or three) external chiral fields $\Phi$. The local counterterm needed to cancel it would be of the form (say in dimensional regularization, where $\epsilon=4-d$ and $l$ is the degree of the pole)

$$
\frac{1}{\epsilon^{l}} \int \mathrm{d}^{4} x \mathrm{~d}^{2} \theta \mathrm{d}^{2} \bar{\theta} \Phi^{2}(x, \theta, \bar{\theta})
$$

but this diagram is identically zero since it is the integral of a chiral quantity over all of superspace. This means that diagrams with two or three chiral fields are always UV convergent and thus $\delta_{m}=\delta_{\lambda}=0$ implying $Z_{m}=Z_{\lambda}=$ 1. At this stage, we do not need to worry about terms like

$$
\int \mathrm{d}^{4} x \mathrm{~d}^{2} \theta \mathrm{d}^{2} \bar{\theta} \Phi \frac{D^{2}}{\square} \Phi
$$

which could be turned into a local F-term by replacing $\mathrm{d}^{2} \bar{\theta} \rightarrow \bar{D}^{2}$ and noting that $\bar{D}^{2} D^{2} \Phi=-\square \Phi$, because such non-local behavior can never arise in the UV.

The situation changes if we are interested in the effective action generating the one particle irreducible diagrams (1PI action) 17. In this context, a UV convergent but IR divergent D-term of the type (56) can arise giving a term that looks like a finite contribution to the superpotential. One explicit example comes from the massless WZ model, where a diagram like Fig. 1 gives a finite contribution to the 1PI effective superpotential:

$$
W_{1 P I}=\int \mathrm{d}^{4} x \mathrm{~d}^{2} \theta \frac{\lambda}{3} \Phi^{3}+\zeta(3) \frac{\lambda^{3} \bar{\lambda}^{2}}{\left(16 \pi^{2}\right)^{2}} \Phi^{3}+\ldots
$$

and the dots indicate that there could be more contributions.

Thus we see that the 1PI superpotential is (by definition) a holomorphic quantity in the fields but not necessarily in the coupling constants. This is an unpleasant state of affairs that suggests that we are not looking at the "right" quantity for our purposes. First of all, since we are interested in gauge theories, the 1PI action constructed via perturbation theory is not well defined since the fields appearing in the Lagrangian are not the correct degrees of freedom at low energy. Even more basically, if we think of the coupling constants as the vacuum expectation values (v.e.v.) of some very 


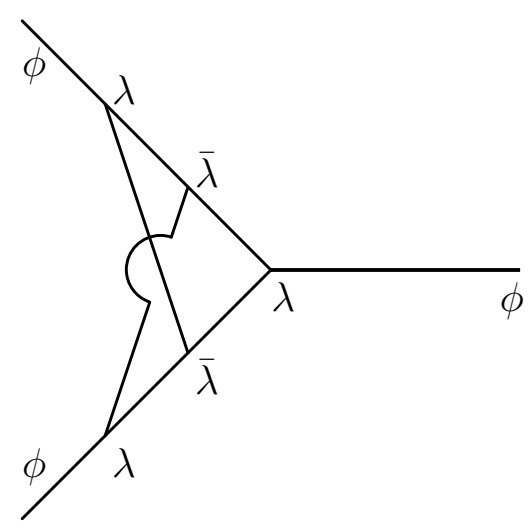

Figure 1: The two-loop contribution to the one-particle effective superpotential.

heavy chiral superfield [18 (as is natural in string theory), then a term like the last one in (57) is not supersymmetric and should be written instead as:

$$
\begin{aligned}
& \int \mathrm{d}^{4} x \mathrm{~d}^{2} \theta \mathrm{d}^{2} \bar{\theta} \lambda^{3} \bar{\lambda}^{2} \Phi^{2} \frac{D^{2}}{\square} \Phi=\int \mathrm{d}^{4} x \mathrm{~d}^{2} \theta \bar{D}^{2}\left(\lambda^{3} \bar{\lambda}^{2} \Phi^{2} \frac{D^{2}}{\square} \Phi\right) \\
= & \int \mathrm{d}^{4} x \mathrm{~d}^{2} \theta\left(\lambda^{3} \Phi^{2} \bar{D}^{2} \bar{\lambda}^{2} \frac{D^{2}}{\square} \Phi+\lambda^{3} \Phi^{2} \bar{D}_{\dot{\alpha}} \bar{\lambda}^{2} \bar{D}^{\dot{\alpha}} \frac{D^{2}}{\square} \Phi-\lambda^{3} \bar{\lambda}^{2} \Phi^{3}\right),
\end{aligned}
$$

where in the last line only the sum of all three terms is manifestly SUSY. In this framework, the whole expression should thus be thought of as a singular D-term.

All this suggests that we forget about the 1PI action and look instead at the Wilsonian action defined as follows: If $S_{\mu_{0}}$ denotes the (bare) action at a scale $\mu_{0}$, then $S_{\mu}$ (for $\mu<\mu_{0}$ ) denotes the action describing the same physics but where the degrees of freedom with momenta between $\mu$ and $\mu_{0}$ have been integrated out. We can be more precise if we restrict our attention to the Wilsonian superpotential because any non-holomorphic dependence on the couplings must be regarded as a (now properly IR regularized) D-term [18. The Wilsonian superpotential $W_{\text {eff }}$ is thus a holomorphic quantity in both fields and coupling constants and it is not perturbatively renormalized. Thus we write:

$$
W_{\text {eff }}=W_{\text {tree }}+W_{\text {non-pert }},
$$

where $W_{\text {tree }}$ is the tree level superpotential and $W_{\text {non-pert }}$ a non-perturbative contribution that cannot be ruled out by the perturbative analysis above. 
Such term is actually absent in the pure WZ model but will be present in many gauge theories and will indeed play a crucial role in all that follows.

Let us make two more remarks. In the case where interacting massless fields are absent, the Wilsonian and the 1PI effective superpotentials coincide. Furthermore, the perturbative non-renormalization of the superpotential can also be proven using holomorphy and the symmetries of the theory as discussed in [18.

Let us now turn to the renormalization of the gauge coupling constant $\tau$ introduced in (44). Since the gauge kinetic term is written as an integral over chiral superspace one might be tempted to argue that the nonrenormalization theorem would apply to this case too. However, this conclusion is wrong because one can write

$$
\int \mathrm{d}^{4} x \mathrm{~d}^{2} \theta \operatorname{tr} W^{\alpha} W_{\alpha}=\int \mathrm{d}^{4} x \mathrm{~d}^{2} \theta \mathrm{d}^{2} \bar{\theta} \operatorname{tr}\left(e^{-V} D^{\alpha} e^{V} W_{\alpha}\right) .
$$

The integrand is not gauge invariant, however the integral is (since it is just a rewriting of a manifestly gauge invariant one) and it is local. Thus, UV divergences of this type are allowed by the non-renormalization theorem and the coupling $\tau$ is not protected from running.

Having established that the coupling constant runs, we can use a piece of information well known from non-supersymmetric theories that states that the $\Theta$ term, being the coefficient of a boundary term, does not get renormalized. Hence, the beta-function for $\tau$ only involves $\operatorname{Re}(\tau)$ and thus cannot be holomorphic. The only exception would be if it were a constant, which would imply that $g$ is renormalized only to one loop. Indeed,

$$
\mu \frac{d}{d \mu} \tau=\frac{\beta}{2 \pi}
$$

where $\beta$ is a real constant, implies

$$
\mu \frac{d}{d \mu} \Theta=0 \text { and } \quad \mu \frac{d}{d \mu} g=-\frac{\beta}{16 \pi^{2}} g^{3} .
$$

An explicit computation shows that this is not the case for the standard coupling constant that in general gets renormalized to all orders. Once again, this suggests that we should introduce a different coupling constant, to be referred to as the Wilsonian coupling, obeying (61), (62). The relation between the ordinary (1PI) and the Wilsonian coupling is of course non-holomorphic [19] and this explains why the two beta-functions disagree already at two loops. Even in this context, it can be shown that the lack of 
holomorphy comes from an IR effect [19] and it is thus consistent to exclude it from the Wilsonian beta-function. From now on $\tau$ will always denote the Wilsonian coupling.

A standard computation then gives:

$$
\left.\beta=\frac{1}{2}(3 T \text { (Adjoint })-\sum_{\rho} T(\rho)\right),
$$

where $T(\rho)$ denotes the index of the irreducible representations $\rho$ in which $\Phi$ transforms, normalized to $T$ (Fundamental) $=1$.

Eq. (61) allows one to introduce a perturbatively RG invariant scale known as the holomorphic scale:

$$
\Lambda^{\beta}=\mu^{\beta} e^{-2 \pi \tau(\mu)}=\mu^{\beta} e^{-\frac{8 \pi^{2}}{g^{2}(\mu)}+i \Theta} .
$$

Consider two theories related to each other by integrating out some field of mass $m$. Because the running is perturbatively only one-loop, the holomorphic scales are easily matched as

$$
\left(\frac{\tilde{\Lambda}}{m}\right)^{\tilde{\beta}}=\left(\frac{\Lambda}{m}\right)^{\beta}
$$

where the tilde quantities refer to the IR theory where the field of mass $m$ has been integrated out.

The importance of the holomorphic scale $\Lambda$ is that it controls the quantum corrections of the observables in the theory, with $\Lambda \rightarrow 0$ corresponding to the classical limit.

Let us now return to the Wilsonian superpotential (59). Notice that one can always write $W_{\text {tree }}=\sum \lambda_{r} X_{r}\left(\Phi_{i}\right)$ for the gauge invariant quantities $X_{r}\left(\Phi_{i}\right)$ introduced in the discussion of the D-terms ${ }^{9}$. The question now is what is the form of $W_{\text {non-pert }}$. One would expect that $W_{\text {non-pert }}$ depends (holomorphically, of course) on the scale $\Lambda$, the couplings $\lambda_{r}$ and the invariants $X_{r}\left(\Phi_{i}\right)$. But, in fact, $W_{\text {non-pert }}$ is independent of the couplings $\lambda_{r}$. This fact is sometimes referred to as the "linearity principle" [20] because it implies that the full superpotential is linear in the couplings.

The linearity principle is less surprising than it seems at first when one realizes that, having ruled out any perturbative dependence on $\lambda_{r}$, any nonperturbative dependence would have to have a singularity as $\lambda_{r} \rightarrow 0$ in

\footnotetext{
${ }^{9}$ In some cases, some of the $X_{r}$ could also be dependent.
} 
some direction in the complex plane. For instance, note that this rules out terms of the type $e^{-1 / \lambda}$ that go to zero only if the origin is approached for $\operatorname{Re}(\lambda)>0$. The gauge coupling $\tau$ is a different story because in this case the real part and the imaginary part enter differently (the real part is always positive) and thus the combination (64) appearing in $\Lambda$ is allowed and it can be generated e.g. by instanton effects.

Having established that $W_{\text {non-pert }}=W_{\text {non-pert }}\left(X_{r}, \Lambda\right)$ it is sometimes possible to completely fix the functional dependence up to some numerical constant by dimensional analysis and symmetry considerations. The most celebrated example of this is the Affleck-Dine-Seiberg superpotential for SQCD, i.e. Supersymmetric $S U\left(N_{c}\right)$ gauge theory with $N_{f}$ quarks $\left(1 \leq N_{f}<N_{c}\right)$ in the fundamental representation [21. The new techniques which are the object of this review allow a more systematic derivation of these non-perturbative corrections to $W_{\text {eff }}$ applicable in more general situations.

What we have seen is that one can write

$$
W_{\text {eff }}=\lambda_{1} X_{1}+\lambda_{2} X_{2}+\cdots+W_{\text {non-pert }}\left(X_{1}, X_{2}, \ldots, \Lambda_{1}, \Lambda_{2}, \ldots\right),
$$

where we have introduced more than one scale $\Lambda_{s}$ to allow for the case where the gauge group is not simple. Let us focus on, say, the dependence on $\lambda_{1}$. Clearly, we can integrate out the field $X_{1}$ at low energy (where the superpotential piece dominates) by solving

$$
\frac{\partial}{\partial X_{1}} W_{\text {eff }}=0
$$

This is tantamount to doing a Legendre transform:

$$
\lambda_{1}=-\frac{\partial}{\partial X_{1}} W_{\text {non-pert }} .
$$

Solving (68) for $X_{1}$ in terms of $\lambda_{1}$ and the other variables and substituting into (66) one obtains $W_{\text {eff }}$ where now the dependence on $\lambda_{1}$ will be complicated because we have integrated out its partner. Thus we can think of $\lambda_{1}$ and $X_{1}$ as forming a canonical pair. One could integrate out all the composite fields $X_{r}$ to give $W_{\text {eff }}$ solely in terms of the couplings $\lambda_{r}$ and the scales $\Lambda_{s}$.

The Legendre transform is clearly invertible and thus, contrary to our "Wilsonian" intuition, as long as we are only interested in F-terms we do not lose any information integrating out a field and, in fact, we can integrate it back in by reversing the procedure:

$$
\left\langle X_{r}\right\rangle=\frac{\partial}{\partial \lambda_{r}} W_{\text {eff }}
$$


Note that this equation will be relevant later in a slightly different way: if we happen to know the v.e.v. of $X_{r}$ in some other way, eq. (69) can be used to determine $W_{\text {eff }}$ as we will discuss in Section 7 .

So far the scales $\Lambda_{s}$ have played a passive role. However, the recent developments depend crucially on the fact that we can also introduce a canonical conjugate for each $\Lambda_{s}$. We can use the perturbatively exact definition of the holomorphic scale (64) to rewrite (for each $s$ ) the gauge kinetic term as a (tree level plus one loop) contribution to the Wilsonian superpotential:

$$
\frac{\tau(\mu)}{16 \pi} \operatorname{tr} W^{\alpha} W_{\alpha} \equiv \beta \log (\Lambda / \mu) S,
$$

where $S$ is the glueball superfield

$$
S=-\frac{1}{32 \pi^{2}} \operatorname{tr}\left(W^{\alpha} W_{\alpha}\right)=\frac{1}{16 \pi^{2}} \operatorname{tr}\left(\lambda^{\alpha} \lambda_{\alpha}+\ldots\right) .
$$

The field $S$ is a chiral superfield whose lowest component is proportional to the gluino bilinear $\operatorname{tr} \lambda^{\alpha} \lambda_{\alpha}$.

The linearity principle still applies in this case. While $S$ does not appear in the original superpotential one can "integrate it in" by solving for $\Lambda$ :

$$
\langle S\rangle=\frac{1}{\beta} \Lambda \frac{d}{d \Lambda} W_{\mathrm{eff}} .
$$

Notice that even without matter fields there is a superpotential for $S$ : the so-called Veneziano-Yankielowicz (VY) superpotential [22]. Consider, as an example, pure $S U(N)$ SYM theory for which $\beta=3 N$. By the assumption of confinement, we expect that all degrees of freedom are massive and thus the effective superpotential at low energy can only be a constant ${ }^{10}$. Dimensional analysis shows that $W_{\text {eff }}=a \Lambda^{3}$ for some numerical constant $a$. Using (72) yields $\langle S\rangle=(a / N) \Lambda^{3}$. From an explicit instanton computation we know that

$$
\langle S\rangle=\Lambda^{3},
$$

that is, $a=N .^{11}$

We can now express the non-perturbative superpotential as a function of $S$ as

$$
W_{\text {non-pert }}=-N S \log \frac{S}{\mu^{3}}+N S .
$$

\footnotetext{
${ }^{10} \mathrm{~A}$ constant superpotential does not break rigid supersymmetry.

${ }^{11}$ The computation of the exact numerical coefficient is a subtle issue. To derive the correct result (73) one needs to perform a computation at weak coupling. We refer to the extensive literature on the subject reviewed in [23]. (See also [24].)
} 


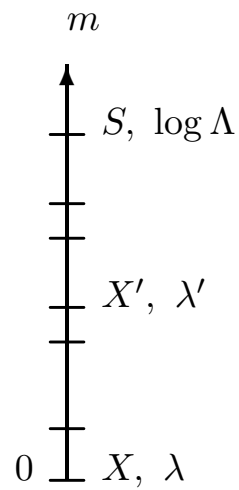

Figure 2: The different energy scales

Sometimes one also writes in a somewhat mixed notation

$$
W_{\text {eff }}=W_{\text {non-pert }}+3 N S \log \frac{\Lambda}{\mu}=N S\left(1-\log \frac{S}{\Lambda^{3}}\right) \equiv W_{V Y},
$$

usually referred to as the VY superpotential. The advantage of this seemingly roundabout notation is that upon minimizing $W_{V Y}$ with respect to $S$ one recovers (73). Thus in the following we will consider $W_{\text {eff }}$ as dependent on $S$ although its "natural" variable should be $\Lambda$.

The general situation is summarized in Table 1

\begin{tabular}{|l|c|c|l|l|l|l|}
\hline Couplings & $\beta_{1} \log \Lambda_{1} / \mu$ & $\beta_{2} \log \Lambda_{2} / \mu$ & $\ldots$ & $\lambda_{1}$ & $\lambda_{2}$ & $\ldots$ \\
\hline Fields & $S_{1}$ & $S_{2}$ & $\ldots$ & $X_{1}$ & $X_{2}$ & $\ldots$ \\
\hline
\end{tabular}

Table 1: Fields and their corresponding couplings

Given the F-terms as functions of one of the variables for each column, one can obtain the dependence on the other variables by performing a Legendre transform.

Let us assume that the mass spectrum for these fields is as given in Fig. 2 Generically, all glueball fields $S$ will be massive whereas we can split the remaining invariants into massive ones, schematically denoted by $X^{\prime}$ with couplings $\lambda^{\prime}$, and massless ones $X$ with couplings $\lambda$. The "most Wilsonian" thing to do would be to express the F-terms as functions of the massless fields $X$, the couplings $\lambda^{\prime}$ and the holomorphic scale $\Lambda$.

What we will see in the following section however is that the new techniques allow us to determine the F-terms as functions of $\lambda, \lambda^{\prime}$ and $S$ instead. Curiously, expressing the F-terms in this set of variables reveals simple dependences on other parameters of the theory such as the number of colors or the number of flavors that are obscured in the initial description. 


\section{Diagrammatic computation of the glueball su- perpotential}

In this section we will describe the first of the two main approaches to the calculation of the glueball superpotential, originally presented in [7]. This approach is essentially perturbative in nature, and we will illustrate it by means of an example. We will follow the discussion of [7] very closely and make some additional simplifications along the way for the sake of clarity.

The computation of [7] is based on the ideas put forward by Dijkgraaf and Vafa in 11, where it was conjectured that the effective superpotential of a gauge theory with matter in the adjoint of $U(N)$ and an arbitrary tree level superpotential $W_{\text {tree }}$ could be fully captured by a bosonic matrix model with a potential coinciding with $W_{\text {tree }}$. More specifically, one is instructed to compute the planar contribution ${ }^{12} \mathcal{F}$ to the free energy of the matrix model:

$$
e^{-\frac{1}{\epsilon^{2}} \mathcal{F}}=\int d M e^{-\frac{1}{\epsilon} W_{\text {tree }}(M)}
$$

where $M$ is a $\hat{N} \times \hat{N}$ hermitian matrix. Taking the large $\hat{N}$ limit while keeping $S=\epsilon \hat{N}$ constant singles out the planar diagrams and yields $\mathcal{F}$ as a function of $S$ and the couplings in $W_{\text {tree. }}$ The effective superpotential for the gauge theory ${ }^{13}$ expressed in terms of these variables is then

$$
W_{\mathrm{eff}}=W_{V Y}+W_{D V},
$$

where $W_{V Y}$ is the same as (175) and

$$
W_{D V}=N \frac{\partial}{\partial S} \mathcal{F}
$$

While the original conjecture was based on a chain of string dualities, the computation of [7] is purely field theoretic, the reduction to a matrix model computation being a direct consequence of $\mathcal{N}=1 \mathrm{SUSY}$ in four dimensions, as we will show below.

Consider the gauge theory described above and for simplicity take $W_{\text {tree }}$ to be a cubic superpotential:

$$
W_{\text {tree }}=\frac{m}{2} \operatorname{tr} \Phi^{2}+\frac{g}{3} \operatorname{tr} \Phi^{3} .
$$

The reader should not confuse $g$ with the gauge coupling that will always be "hidden" in $\Lambda$ in the following. Note that renormalizability is not an

\footnotetext{
${ }^{12}$ We will see in detail how this is done.

${ }^{13}$ For simplicity we limit ourselves to discuss the unbroken phase at this stage.
} 
issue here, since on the one hand the computation we will review is not sensitive to the degree of the superpotential, and on the other hand the theory we consider could be thought of as an effective field theory of an underlying renormalizable theory. Thus, the generalization to an arbitrary superpotential is straightforward.

Thought as a polynomial in a complex variable $z$, the superpotential $W_{\text {tree }}(z)=\frac{m}{2} z^{2}+\frac{g}{3} z^{3}$ has two extrema ${ }^{14}: z=0$ and $z=-m / g$. The classical vacua are constructed by setting each (complex) eigenvalue of the $N \times N$ matrix $\langle\Phi\rangle$ equal to one of the two extrema. This generically breaks the gauge symmetry from $U(N)$ to $U(p) \times U(N-p)$. In this section we will study only the unbroken $U(N)$ phase, i.e. we set the v.e.v. of $\Phi$ identically to zero.

The goal is to compute the effective superpotential $W_{\text {eff }}(S, g, m)$, as discussed at the end of Section 3. Apart from the non-perturbative VY piece (75) already present in the pure gauge theory, the only contributions to $W_{\text {eff }}$ come from the perturbative evaluation of the path integral over the chiral superfield in a constant background for the vector superfield.

Note that this is not in contradiction to the non-renormalization theorem, since when one integrates out $S$, all terms in $W_{\text {eff }}$ appear as powers of the holomorphic scale $\Lambda$. Thus it is true that $W_{\text {eff }}$ is a perturbative series in the coupling $g$, but each term is accompanied by a positive power of the essentially non-perturbative object $\Lambda$ coming from the gauge sector. Were we to decouple the gauge sector from the chiral superfield, we would have to take $\Lambda$ to zero, which would make $W_{\text {eff }}$ vanish.

We choose the simplest possible background for our purposes, i.e. a constant gaugino $\lambda_{\alpha}$ and a vanishing gauge field, $A_{\mu}=0$. In order for the above background to satisfy trivially the equations of motion, we can set to zero the complex conjugate gaugino, $\bar{\lambda}_{\dot{\alpha}}=0$, employing the usual trick of thinking of $\lambda_{\alpha}$ and $\bar{\lambda}_{\dot{\alpha}}$ as independent objects. This is justified as long as we are interested in $\bar{\lambda}_{\dot{\alpha}}$ independent quantities such as the F-terms. ${ }^{15}$

In superfield terms, the background simply reads:

$$
V=-\sqrt{2} i \bar{\theta}^{2} \theta \lambda, \quad W_{\alpha}=-\sqrt{2} i \lambda_{\alpha} .
$$

\footnotetext{
${ }^{14}$ Here and in the following we make an abuse of notation replacing $\Phi$ by $z$ in $W_{\text {tree }}$ but refraining from taking the trace.

${ }^{15}$ It is possible to take a different approach and keep all generality in the background and in the superpotential, while using the full power of supergraph calculus (R.A. thanks D. Zanon for an interesting discussion on this issue). This would certainly be appropriate in an exhaustive review, however here we prefer not to introduce the more advanced material required for this treatment and instead we stick to a more intuitive approach as in 7 .
} 
There is a further restriction to be obeyed by the constant gluino background - a SUSY variation of the gauge field, $\delta A_{\mu}=i \bar{\xi} \bar{\sigma}_{\mu} \lambda$, is required to be pure gauge, thus implying that the matrix valued $\lambda_{\alpha}$ satisfy:

$$
\left\{\lambda_{\alpha}, \lambda_{\beta}\right\}=0 .
$$

In this background, covariant spacetime derivatives reduce to ordinary partial derivatives, the gluino superfield $W_{\alpha}$ is constant (and thus covariantly constant) and, most importantly, traces of more than two $W_{\alpha}$ vanish. This comes about because eq. (81) implies that, for instance, $\operatorname{tr} W_{\alpha} W_{\beta} W_{\gamma}$ must be totally antisymmetric in the spinorial indices, which take only two values. A further and less obvious consequence of (81) is that the glueball superfield $S$, as given in (71), satisfies $S^{h}=0$, where $h$ is the dual Coxeter number of the gauge group. This issue will be discussed in more detail in the next section, but it should be clear already at this stage that $S$ is nilpotent because there are only a finite number of Grassmann variables in $W_{\alpha}$.

We want to compute the holomorphic contribution to the partition function:

$$
Z=\int \mathcal{D} \Phi \mathcal{D} \bar{\Phi} e^{i S}
$$

where the action is:

$S=\int \mathrm{d}^{4} x \mathrm{~d}^{2} \theta \mathrm{d}^{2} \bar{\theta} \operatorname{tr}\left(e^{-V} \bar{\Phi} e^{V} \Phi\right)+\int \mathrm{d}^{4} x \mathrm{~d}^{2} \theta W_{\text {tree }}(\Phi)+\int \mathrm{d}^{4} x \mathrm{~d}^{2} \bar{\theta} \bar{W}_{\text {tree }}(\bar{\Phi})$.

In the kinetic term we have written the adjoint action on $\Phi$ explicitly, i.e. in terms of $N \times N$ matrices. Equivalently, one can define the adjoint action of $V$ on $\Phi$ as $\operatorname{Ad} V \Phi=[V, \Phi]$. One can then exponentiate it by formal power series and check that:

$$
\begin{aligned}
e^{\operatorname{Ad} V} \Phi & =1+\operatorname{Ad} V \Phi+\frac{1}{2}(\operatorname{Ad} V)^{2} \Phi+\ldots \\
& =1+[V, \Phi]+\frac{1}{2}[V,[V, \Phi]]+\cdots=e^{V} \Phi e^{-V}
\end{aligned}
$$

Both ways of writing this expression will be used.

As far as the computation of the F-terms is concerned, we can set $\bar{g}=0$ and $\bar{m}=1$ independently of $g$ and $m$, since they are not going to enter any holomorphic quantity ${ }^{16}$. In this way the overall $\bar{\Phi}$ dependence will be

\footnotetext{
${ }^{16}$ Notice that setting $\bar{m}=1$ will imply that some of the intermediate formulas that follow will not be manifestly dimensionally correct. The final (holomorphic) result will of course be.
} 
quadratic. We can thus write:

$$
Z=\int \mathcal{D} \Phi e^{i \int \mathrm{d}^{4} x \mathrm{~d}^{2} \theta W_{\text {tree }}(\Phi)} \int \mathcal{D} \bar{\Phi} e^{i S_{0}}
$$

where

$$
S_{0}=\int \mathrm{d}^{4} x \mathrm{~d}^{2} \theta \mathrm{d}^{2} \bar{\theta} \operatorname{tr}\left(e^{-V} \bar{\Phi} e^{V} \Phi\right)+\int \mathrm{d}^{4} x \mathrm{~d}^{2} \bar{\theta} \frac{1}{2} \operatorname{tr} \bar{\Phi}^{2}
$$

We start by evaluating the (Gaussian) path integral over $\bar{\Phi}$.

The action $S_{0}$ can be reformulated by defining:

$$
\tilde{\Phi} \equiv e^{-V} \bar{\Phi} e^{V}=e^{-\operatorname{Ad} V} \bar{\Phi}=\bar{\Phi} e^{\operatorname{Ad} V} .
$$

Using the covariant fermionic derivatives defined in (41), where $V$ should be intended in the adjoint representation (i.e. $\nabla_{\alpha}=e^{-\operatorname{Ad} V} D_{\alpha} e^{\operatorname{Ad} V}$ ) the fields now obey $\bar{\nabla}_{\dot{\alpha}} \Phi=0$ and $\nabla_{\alpha} \tilde{\Phi}=0$. More importantly, the path integral over $\bar{\Phi}$ can be safely replaced by a path integral over $\tilde{\Phi}$, since no anomalies can arise, $\Phi$ being in the adjoint representation.

Finally, to complete the square, we need to turn the integral over antichiral superspace into an integral over full superspace:

$$
\begin{aligned}
S_{0} & =\int \mathrm{d}^{4} x \mathrm{~d}^{2} \theta \mathrm{d}^{2} \bar{\theta} \operatorname{tr}(\tilde{\Phi} \Phi)+\int \mathrm{d}^{4} x \mathrm{~d}^{2} \bar{\theta} \frac{1}{2} \operatorname{tr} \tilde{\Phi}^{2} \\
& =\int \mathrm{d}^{4} x \mathrm{~d}^{2} \theta \mathrm{d}^{2} \bar{\theta} \operatorname{tr}\left(\tilde{\Phi} \Phi+\frac{1}{2} \tilde{\Phi} \frac{1}{\nabla^{2}} \tilde{\Phi}\right)
\end{aligned}
$$

where we have used the fact that $\int \mathrm{d}^{2} \theta$ can be replaced by $\nabla^{2}$ when inside the spacetime integral and the trace.

Note that $\nabla^{2}$ is not invertible in all of superspace (for instance it vanishes on covariantly antichiral superfields like $\tilde{\Phi})$, but we can define its inverse up to an element of its kernel. In the literature, it is also often written equivalently either as $\left(\bar{\nabla}^{2} \nabla^{2}\right)^{-1} \bar{\nabla}^{2}$ or as $\bar{\nabla}^{2}\left(\nabla^{2} \bar{\nabla}^{2}\right)^{-1}$. The first expression is obviously the left-inverse of $\nabla^{2}$, while the second is the right-inverse, and again they coincide up to an element of the kernel as can be checked by conjugation.

Completing the square, the action can thus be rewritten as:

$$
S_{0}=\int \mathrm{d}^{4} x \mathrm{~d}^{2} \theta \mathrm{d}^{2} \bar{\theta} \frac{1}{2}\left[\operatorname{tr}\left(\tilde{\Phi}+\nabla^{2} \Phi\right) \frac{1}{\nabla^{2}}\left(\tilde{\Phi}+\nabla^{2} \Phi\right)-\operatorname{tr}\left(\Phi \nabla^{2} \Phi\right)\right] .
$$


Now the first piece is trivially a quadratic integral on antichiral superspace for the shifted antichiral superfield $\tilde{\Phi}^{\prime}=\tilde{\Phi}+\nabla^{2} \Phi$, while the second piece can be turned into an integral over chiral superspace:

$$
S_{0}=\int \mathrm{d}^{4} x \mathrm{~d}^{2} \bar{\theta} \frac{1}{2} \operatorname{tr}\left(\tilde{\Phi}^{\prime}\right)^{2}-\int \mathrm{d}^{4} x \mathrm{~d}^{2} \theta \frac{1}{2} \operatorname{tr}\left(\Phi \bar{\nabla}^{2} \nabla^{2} \Phi\right) .
$$

The path integral over $\tilde{\Phi}$ can be harmlessly turned into a path integral over $\tilde{\Phi}^{\prime}$, which is Gaussian and independent of the background, thus contributing just a constant to the partition function $Z$.

Returning to (85), we are left with a chiral path integral over $\Phi$. Contrary to the integral over $\bar{\Phi}$, the action here contains interactions, forcing us to a perturbative evaluation. We will however encounter a remarkable simplification due to supersymmetry which will lead us to consider a matrix model.

To obtain the explicit form of the propagator for $\Phi$ we need to expand the expression $\bar{\nabla}^{2} \nabla^{2} \Phi$. Recalling the definitions, we have that (in this simple background):

$\bar{\nabla}^{2} \nabla^{2} \Phi=\bar{D}^{2} e^{-\operatorname{Ad} V} D^{2} e^{\operatorname{Ad} V} \Phi=-\square \Phi-\operatorname{Ad} W^{\alpha} D_{\alpha} \Phi=-\square \Phi-\left\{W^{\alpha}, D_{\alpha} \Phi\right\}$,

where in this case the adjoint action gives rise to an anticommutator.

The path integral (85) is thus reduced to the following chiral path integral:

$$
Z^{\prime}=\int \mathcal{D} \Phi e^{i \int \mathrm{d}^{4} x \mathrm{~d}^{2} \theta \frac{1}{2} \operatorname{tr} \Phi\left(\square+\operatorname{Ad} W^{\alpha} D_{\alpha}+m\right) \Phi+\frac{g}{3} \operatorname{tr} \Phi^{3}} .
$$

The quantity $Z^{\prime}$ defined above is the purely holomorphic part of the full partition function (82), i.e. the piece that depends only on the holomorphic couplings and on the holomorphic background $\lambda_{\alpha}$. We can thus associate it with the coupling dependent part of the effective superpotential:

$$
Z^{\prime} \equiv e^{i \int \mathrm{d}^{4} x \mathrm{~d}^{2} \theta W_{D V}(S, g, m)}
$$

The procedure outlined above is quite general, and it applies to any group, matter content, and tree level superpotential.

There are however two important points to make. The first one is that, since $Z^{\prime}$ is the partition function obtained by integrating over the matter fields, there is no hope of getting the piece of the effective superpotential pertaining to the pure gauge low-energy dynamics, i.e. the VenezianoYankielowicz piece (75), in this way. This piece is independent of the matter couplings $g$ and $m$ and cannot arise from a perturbative evaluation of (92). 
The second point is that the perturbative evaluation of (92) has to take into account the fact that the classical gluino background satisfies (81). As said before, this implies that the gauge invariant gluino bilinear $S$ is nilpotent, i.e. $S^{h}=0$. For instance, in the $U(N)$ case considered above, $W_{D V}(S, g, m)$ would have a truncated Taylor expansion which stops at $S^{N}$. Of course, this makes no sense when $S$ is considered as an effective chiral superfield, and we will indeed find superpotentials $W_{D V}(S, g, m)$ with an infinite Taylor expansion, of which the first $h-1$ terms coincide with the ones computed by the method above.

In some cases however, one can compute $W_{D V}(S, g, m)$ to arbitrary powers of $S$ without any obstacle. This is possible for instance in the case considered here because the dependence on $N$ factorizes in front of $W_{D V}$, as stated in (78). Since $\mathcal{F}$ is independent of $N$ we can compute its power series expansion to arbitrary order by formally considering $N$ large and then substitute into (78) with the actual value of $N$. In other cases discrepancies with exact field theory results can show up at order $h$, as discussed in 25].

We now proceed to evaluate perturbatively (92) and to show that in the case of $U(N)$ with adjoint matter, it reduces to the evaluation of a matrix model integral.

First of all, it will be useful to go to momentum space. Following [7], we introduce not only the usual (bosonic) 4-momentum $p_{\mu}$, but also a fermionic momentum $\pi^{\alpha}$ conjugate to $\theta_{\alpha}$. In the quadratic part of the action in (92), we Wick rotate to Euclidean space and Fourier transform in both the bosonic and fermionic coordinates by substituting:

$$
\square \rightarrow-\square_{E u c l} \rightarrow p^{2}, \quad D_{\alpha} \rightarrow \pi_{\alpha} .
$$

It may be surprising that we do not include a factor of $i$ in the last definition but this is consistent with our choice of hermiticity for $D_{\alpha}$.

We now turn to the propagator $\left\langle\Phi_{c}^{a}(p, \pi) \Phi_{d}^{b}(-p,-\pi)\right\rangle$ for $\Phi$. In the following $\Phi$ is thought of as a $N \times N$ matrix and the roman indices $a, b, \ldots$ run from 1 to $N$. Looking at the kinetic term in (92) we read off the inverse propagator:

$$
\Gamma_{c d}^{a b}(p, \pi)=\left(p^{2}+m\right) \delta_{d}^{a} \delta_{c}^{b}+\left(W_{d}^{\alpha a} \delta_{c}^{b}-W_{c}^{\alpha b} \delta_{d}^{a}\right) \pi_{\alpha} .
$$

We can write the propagator itself using a Schwinger parameter $s$ :

$$
\Delta(p, \pi)=\int_{0}^{\infty} d s e^{-s\left(p^{2}+m+\operatorname{Ad} W^{\alpha} \pi_{\alpha}\right)},
$$



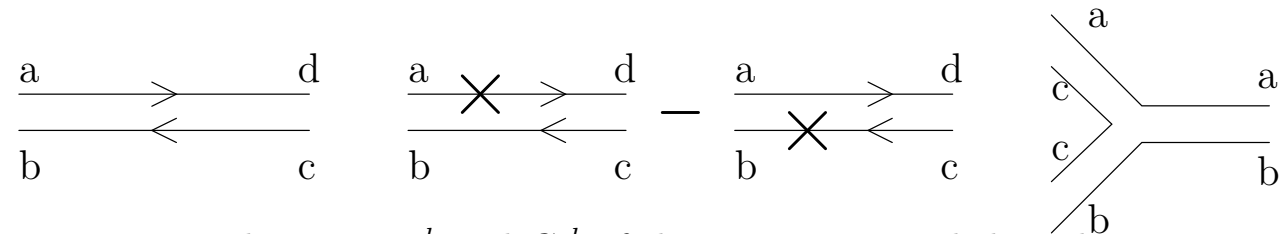

Figure 3: The parts $1_{c d}^{a b}$ and $G_{c d}^{a b}$ of the propagator and the cubic vertex.

where, as usual, the exponential is defined by power series expansion ${ }^{17}$. Actually, since $\pi_{\alpha}$ is a Grassmann two-component spinor, the expansion stops at the second order. Thus we have:

$$
e^{-s G}=1-s G+\frac{1}{2} s^{2} G^{2},
$$

with

$$
1_{c d}^{a b} \equiv \delta_{d}^{a} \delta_{c}^{b}, \quad G_{c d}^{a b} \equiv\left(W_{d}^{\alpha a} \delta_{c}^{b}-W_{c}^{\alpha b} \delta_{d}^{a}\right) \pi_{\alpha} .
$$

In order to compute $Z^{\prime}$ we have to evaluate vacuum diagrams of the theory defined by the action in (92). Because the matter field $\Phi$ is in the adjoint, the diagrams can be written using the double line notation [26] (see Fig. 3). The identity in (97) is represented by two lines with opposite orientations, while the $G$ and $G^{2}$ terms are represented by the same lines with one or two $W^{\alpha}$ insertions respectively. The cubic vertex, being a trace, is represented by three lines joining three different two-line propagators.

Having established the above, one can assign a topology to any given graph in the usual way. The graph represents a discretization of a twodimensional closed surface over which it can be drawn without any twisting or intersection. Call $V$ the number of vertices, $P$ the number of propagators and $F$ the number of faces i.e. regions encircled by a line, including the external one. Then the Euler characteristic of the surface is given by:

$$
\chi=V-P+F,
$$

and it is a topological invariant. For instance, a sphere has Euler characteristic $\chi=2$ and a torus has $\chi=0$. Actually, every handle one "adds" to the surface reduces $\chi$ by 2 , so that the following formula is valid for closed orientable surfaces:

$$
\chi=2-2 h,
$$

\footnotetext{
${ }^{17}$ Notice that $\left\{W^{\alpha}, \pi_{\alpha} \Phi\right\}=\left[W^{\alpha} \pi_{\alpha}, \Phi\right]$.
} 
where $h$ here is the number of handles, also referred to as the genus of the surface.

Allowing for open and/or unoriented surfaces, the Euler characteristic can take odd values (but always keeping $\chi \leq 2$ for a single surface). For instance, $\chi=1$ for a disk (open, oriented surface) and for the projective plane (closed, unoriented surface).

An important result that is obtained even before starting any computation is that only planar graphs contribute to the superpotential. This can be understood as follows. A generic (connected) vacuum diagram has $V$ vertices and $P$ propagators. Every vertex carries with it an integral over chiral (coordinate) superspace, while every propagator, after Fourier transforming it, carries an integral over chiral momentum superspace. All but one of the coordinate superspace integrals give delta functions enforcing momentum conservation (both bosonic and fermionic) at every vertex, the remaining overall chiral superspace integral being the one which appears in the effective action in (93). Solving for the delta functions, one is left with $P-V+1 \equiv L$ momentum superspace (loop) integrals. So far this is just ordinary loop counting applied also to the Grassmann momenta. Thus one has to integrate over $2 L$ Grassmannian momenta $\pi_{\alpha}$. In the integrand, these momenta necessarily appear in bilinears together with the gluino background $W^{\alpha}$ because of the specific form of the propagator (96). Since traces of more than two $W^{\alpha}$ vanish due to (81), the result of the integration is nonzero only if we are able to write at least $L$ traces. Remembering now that the number of traces coincides with the number of closed lines, or "faces" of the graph, we get the following constraint on the graph's topology:

$$
F \geq L=P-V+1 \quad \Rightarrow \quad \chi \geq 1 .
$$

This means that in the case of the matter field in the adjoint of $U(N)$, we are concerned only with graphs with the topology of a sphere, that is planar graphs. All the other ones are exactly zero already at finite $N$. In other words, planarity is an exact consequence of supersymmetry, and not a leading order approximation due to a large $N$ limit.

When open or unoriented graphs have to be taken into account, also graphs with one boundary or one cross cap give a non-vanishing result. Graphs with boundaries arise in theories with matter fields in the fundamental representation, whose propagator has only one color line (possibly supplemented by a flavor line), while unoriented graphs arise in theories where the gauge group is $S O(N)$ or $S p(N)$. Note also that graphs of higher genera become important when the theory is coupled to gravity, in the background of which they no longer vanish. 
Let us now compute in detail one diagram in order to give a feeling of how a field theory computation can be reduced to a matrix model one.

Let us compute the "stop sign" diagram of Fig. 4.

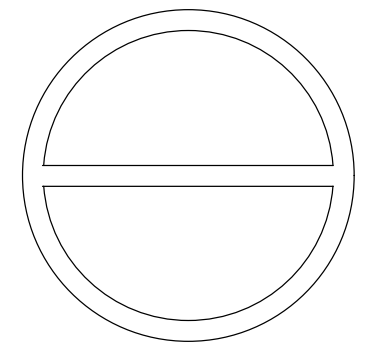

Figure 4: The stop sign diagram.

This diagram yields:

$$
\frac{g^{2}}{6} \int \frac{\mathrm{d}^{4} p_{1}}{(2 \pi)^{4}} \frac{\mathrm{d}^{4} p_{2}}{(2 \pi)^{4}} \mathrm{~d}^{2} \pi_{1} \mathrm{~d}^{2} \pi_{2} \Delta_{c d}^{a b}\left(p_{1}, \pi_{1}\right) \Delta_{a f}^{e d}\left(p_{2}, \pi_{2}\right) \Delta_{e b}^{c f}\left(-p_{1}-p_{2},-\pi_{1}-\pi_{2}\right)
$$

The overall factor of $\frac{g^{2}}{6}$ is understood as follows. We have a $\frac{1}{2}$ from the Taylor expansion to second order of the exponential of the interaction, a $(g / 3)^{2}$ from the two interaction vertices, and a 3 from the number of independent ways one can contract the $\Phi$ to give a planar graph ${ }^{18}$.

In terms of the three Schwinger parameters, we can rewrite the diagram as:

$$
\frac{g^{2}}{6} \int d s_{1} d s_{2} d s_{3} e^{-m\left(s_{1}+s_{2}+s_{3}\right)} I_{\text {bose }} I_{\text {fermi }} .
$$

The bosonic integral is straightforward, since it does not imply any group theory factors. It is given by:

$$
\begin{aligned}
I_{\text {bose }} & =\int \frac{\mathrm{d}^{4} p_{1}}{(2 \pi)^{4}} \frac{\mathrm{d}^{4} p_{2}}{(2 \pi)^{4}} e^{-s_{1} p_{1}^{2}-s_{2} p_{2}^{2}-s_{3}\left(p_{1}+p_{2}\right)^{2}} \\
& =\frac{1}{(4 \pi)^{4}} \frac{1}{\left(s_{1} s_{2}+s_{1} s_{3}+s_{2} s_{3}\right)^{2}} .
\end{aligned}
$$

The computation of the fermionic integral is more involved, since the inte-

\footnotetext{
${ }^{18}$ There are also three more contractions that do not yield a planar graph and thus vanish according to the above argument. This explains the discrepancy between (102) and the factor that would arise in a single component $\phi^{3}$ theory.
} 
grand involves traces over the gauge group:

$$
I_{\text {fermi }}=\int d^{2} \pi_{1} d^{2} \pi_{2}\left(e^{-s_{1} \mathrm{Ad} W^{\alpha} \pi_{1 \alpha}}\right)_{c d}^{a b}\left(e^{-s_{2} \mathrm{Ad} W^{\alpha} \pi_{2 \alpha}}\right)_{a f}^{e d}\left(e^{s_{3} \operatorname{Ad} W^{\alpha}\left(\pi_{1 \alpha}+\pi_{2 \alpha}\right)}\right)_{e b}^{c f}
$$

The integral over the fermionic momenta is saturated only if we bring down two $\pi_{1}$ and two $\pi_{2}$ :

$$
\int d^{2} \pi_{1} d^{2} \pi_{2} \pi_{1}^{2} \pi_{2}^{2}=1
$$

The result thus gives an overall fourth power of the gluino $W^{\alpha}$. These four gluinos have to be inserted at some point of the three color loops of the diagram. Since we cannot put more than two gluinos on the same line (otherwise the trace would vanish), the two choices we are left with are either to put two $W^{\alpha}$ on two color loops and leave the third loop without insertion, or to put two $W^{\alpha}$ on one loop and one each in the two remaining color loops.

The latter combination leads to a term including $\operatorname{tr} W^{\alpha} \operatorname{tr} W_{\alpha}$, which is the effective kinetic term for the decoupled $U(1)$. Terms of this type are interesting in the case of broken gauge group but here we focus only on the effective superpotential for the gluino bilinear $S$, i.e. we are concerned only with those combinations where either two $W^{\alpha}$ or none are inserted in each color loop. The $U(1)$ couplings will be briefly discussed in Section 7 .

It is easy to work out all the insertions pictorially, instead of developing the propagators by brute force. One can then realize that two different kinds of diagrams contribute. The first, depicted on the left of Fig. [5] arises when
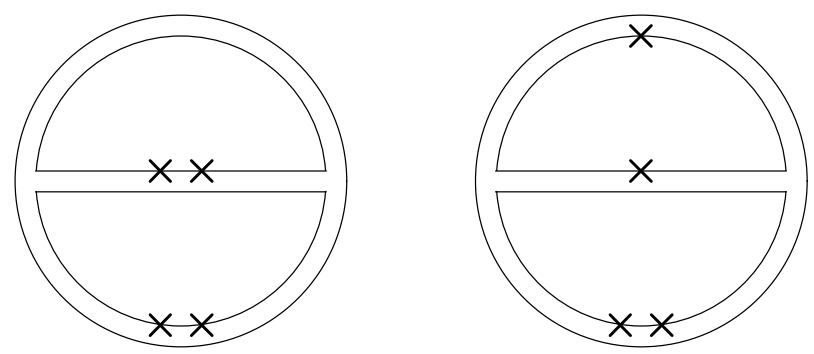

Figure 5: The stop sign with two $W_{\alpha}$ insertions at two of the propagators (left) and the stop sign with one $W_{\alpha}$ insertion at two of the propagators and one insertion of two $W_{\alpha}$ at the third propagator (right).

two propagators have two insertions and the third has none ${ }^{19}$. Diagrams of

\footnotetext{
${ }^{19}$ At this point it is important not to confuse propagators and color loops - propagators
} 
these type contribute to $I_{\text {fermi }}$ a quantity:

$$
3 N\left(\operatorname{tr} W^{\alpha} W_{\alpha}\right)^{2}\left(\frac{1}{2} s_{1}^{2} \frac{1}{2} s_{2}^{2}+\frac{1}{2} s_{1}^{2} \frac{1}{2} s_{3}^{2}+\frac{1}{2} s_{2}^{2} \frac{1}{2} s_{3}^{2}\right),
$$

where the factor of $N$ is given by the color loop without insertions, $\operatorname{tr} 1=N$, while the factor of 3 comes from the choice of this color loop.

The second kind of diagram, depicted on the right of Fig. 5, arises when one propagator carries two insertions and the other two one each. The contribution from these diagrams is:

$$
3 N\left(\operatorname{tr} W^{\alpha} W_{\alpha}\right)^{2}\left(\frac{1}{2} s_{1}^{2} s_{2} s_{3}+s_{1} \frac{1}{2} s_{2}^{2} s_{3}+s_{1} s_{2} \frac{1}{2} s_{3}^{2}\right),
$$

the numerical prefactor having the same origin as above.

Summing these contributions, we get, using (71):

$$
I_{\text {fermi }}=3 N(4 \pi)^{4} S^{2}\left(s_{1} s_{2}+s_{1} s_{3}+s_{2} s_{3}\right)^{2} .
$$

Comparing now with the result of the bosonic integral (104), we observe the striking fact that the $s_{i}$ dependent part of the numerator and the denominator exactly cancel ${ }^{20}$, leaving:

$$
I_{\text {bose }} I_{\text {fermi }}=3 N S^{2}
$$

that is, a result independent of the Schwinger parameters $s_{i}$ !

Plugging this back into (103), the integral over $s_{1}, s_{2}$ and $s_{3}$ becomes trivial:

$$
\int d s_{1} d s_{2} d s_{3} e^{-m\left(s_{1}+s_{2}+s_{3}\right)}=\frac{1}{m^{3}} .
$$

In other words, all that is left from the propagators is a contribution of $\frac{1}{m}$ each, exactly what one would expect from a zero-dimensional field theory, i.e. from a matrix model. In particular, note that the result is finite. This is non-trivial since after all we are dealing with a two-loop computation.

The final result for the stop sign diagram is thus:

$$
\frac{1}{6} \frac{g^{2}}{m^{3}} 3 N S^{2}
$$

To order $g^{2}$ there is only another diagram, shown in Fig. 6] contributing to the effective superpotential. We leave it as an exercise to check that, after

can carry at most two insertions because their expansion stops at second order.

${ }^{20}$ Curiously, this works only in four spacetime dimensions, as the overall powers of $s_{i}$ in the numerator are determined by the dimension of the Weyl spinor, whereas the ones of the denominator are determined by the dimension of spacetime. 


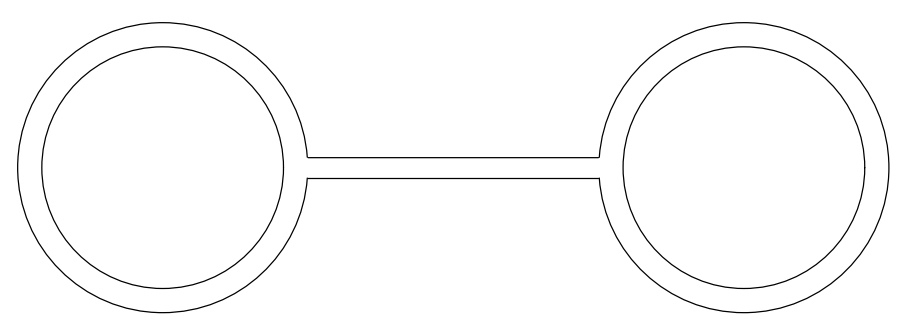

Figure 6: The drum-bell diagram.

all the $W_{\alpha}$ insertions are made, it contributes a quantity

$$
\frac{1}{2} \frac{g^{2}}{m^{3}} 3 N S^{2}
$$

Summing (112) and (113) and recalling the overall minus sign coming from the Wick rotation we get the full contribution to the effective superpotential to this order:

$$
W_{D V}=-2 N \frac{g^{2}}{m^{3}} S^{2}+\ldots
$$

In all generality, we can argue as above that for a planar diagram with $F$ color loops, the $S^{L} \equiv S^{F-1}$ term will have a prefactor of $N$ from the color loop without insertions and a factor of $F$ from the choice of such a loop:

$$
I_{\text {bose }} I_{\text {fermi }}=F N S^{F-1}=N \frac{\partial}{\partial S} S^{F} .
$$

The numerical and coupling dependent prefactor will depend on the interaction vertex and on the combinatorics. That the $s_{i}$ dependence completely cancels in the above expression is less trivial to see, but can actually be proven in all generality introducing a new set of $2 L$ auxiliary Grassmann coordinates to enforce the fact that one allows for at most two insertions of $W^{\alpha}$ in any color loop. We preferred here to present a sample computation, and we refer the interested reader to the original derivation [7.

The relevant question now is whether we can define a matrix model from which to extract all the information we need to compute the effective superpotential of the gauge theory. The answer turns out to be quite straightforward.

Let us look at the following matrix model:

$$
\mathcal{Z}=\int d M e^{-\frac{1}{\epsilon} \operatorname{tr}\left(\frac{m}{2} M^{2}+\frac{g}{3} M^{3}\right)}
$$


Here $M$ is an $\hat{N} \times \hat{N}$ hermitian matrix, where $\hat{N}$ is not related to the $N$ of the gauge theory. Actually, for the time being the only explicit link between the matrix model and the gauge theory is that the potential of the matrix model is given by the tree level superpotential $W_{\text {tree }}$ of the gauge theory, after substituting $\Phi$ for $M$. Otherwise, note for instance that the matrix model is purely bosonic. The parameter $\epsilon$ is introduced for later convenience, and plays the role of an overall coupling constant.

Strictly speaking the potential in (116) is not well defined because it does not have a minimum. This should not be however a matter of worry, since we will use (116) to define a (formal) perturbative expansion around its local minimum (recall that we are interested in the case where, on the gauge theory side, the v.e.v. of $\Phi$ does not break the gauge group).

Indeed, we write:

$$
\begin{aligned}
\mathcal{Z} & =\int d M e^{-\frac{1}{\epsilon} \operatorname{tr}\left(\frac{m}{2} M^{2}\right)} \sum_{n=0}^{\infty} \frac{(-1)^{n}}{n !}\left(\frac{1}{\epsilon} \frac{g}{3} \operatorname{tr} M^{3}\right)^{n} \\
& =\sum_{n=0}^{\infty} \frac{(-1)^{n}}{n !}\left\langle\left(\frac{1}{\epsilon} \frac{g}{3} \operatorname{tr} M^{3}\right)^{n}\right\rangle .
\end{aligned}
$$

In the second line above, we have used the correlation functions of the "free" matrix model, that is the one given by a purely quadratic potential. The free propagator is given by:

$$
\left\langle M_{b}^{a} M_{d}^{c}\right\rangle=\frac{\epsilon}{m} \delta_{d}^{a} \delta_{b}^{c}
$$

and can also be represented by a double line as in the previous set up. Note however that here the roman indices run between 1 and $\hat{N}$.

The vacuum diagrams of the interacting theory are represented by the correlation functions (117) of the free theory. Every vertex is multiplied by a factor of $g / \epsilon$. Hence a generic diagram will give a contribution of:

$$
c\left(\frac{\epsilon}{m}\right)^{P}\left(\frac{g}{\epsilon}\right)^{V} \hat{N}^{F},
$$

where $c$ is a numerical coefficient including the powers of $\frac{1}{3}$ coming from the vertices, the coefficients of the Taylor expansion of the exponential and the various combinatorics. Again, $F$ is the number of index loops, i.e. traces.

The matrix model simplifies greatly when one takes the large $\hat{N}$ limit. In order to do this properly, let us take simultaneously the limit $\epsilon \rightarrow 0$, while keeping the combination:

$$
\epsilon \hat{N}=S
$$


constant (it is of course not an accident that the constant is called $S$, we will shortly see below why).

We now solve for $\hat{N}$ in (119), and recalling the expression for the Euler characteristic (99), we obtain for the graph:

$$
c \epsilon^{-\chi}\left(m^{-P} g^{V} S^{F}\right) \text {. }
$$

The expression between parenthesis is finite in the limit, and when $\epsilon \rightarrow 0$ the planar diagrams $(\chi=2)$ will clearly dominate, the other ones being suppressed by powers of $1 / \hat{N}^{2}$.

Reexpressing the partition function for the matrix model as:

$$
\mathcal{Z}=e^{-\frac{1}{\epsilon^{2}} \mathcal{F}_{\chi=2}-\mathcal{F}_{\chi=0}-\epsilon^{2} \mathcal{F}_{\chi=-2}+\ldots},
$$

we observe that the result of computing all planar diagrams is contained in the planar free energy $\mathcal{F} \equiv \mathcal{F}_{\chi=2}(S, g, m)$. For instance, the contribution to $\mathcal{F}$ from the "stop sign" diagram considered before, is:

$$
-\frac{1}{6} \frac{1}{m^{3}} g^{2} S^{3}
$$

where the power of $S$ is now simply the number of color index loops. The cubic matrix model can be solved exactly in the planar limit, as first shown in [10] and in Appendix B we indeed show how to obtain the complete $\mathcal{F}$.

Recalling the expression (115) derived on the gauge theory side, the only non-trivial pieces of informations to compute are the numerical factors in front of every term in the perturbative expansion in $S$. These are precisely the numbers contained in the function $\mathcal{F}$. Hence the effective superpotential of the gauge theory is given in terms of the matrix model planar free energy by:

$$
W_{D V}(S, g, m)=N \frac{\partial}{\partial S} \mathcal{F}(S, g, m)
$$

Let us underscore again the important point - planarity is achieved through the large $\hat{N}$ limit on the matrix model side, but is an exact feature at finite $N$ on the field theory side. The reason is that on the field theory side non-planar diagrams necessarily include traces of more than two gluino superfields, and these vanish exactly.

Furthermore, the only way $N$ enters in the effective superpotential is through a multiplicative factor in (124). Thus, we see that, even if on the gauge theory side we should trust the effective superpotential only up to the term $S^{N}$, the matrix model instructs us to go on and compute any diagram in the same way. This works in this case because the functional form of 
the superpotential does not change when $N$ is varied, and thus one could also take a large value of $N$ on the gauge theory side. This generalization must of course rely on considering $S$ a dynamical effective field (the glueball superfield).

We have already stressed that the perturbative approach does not give us a systematic way to compute the pure gauge part of the effective superpotential, namely the Veneziano-Yankielowicz superpotential for a pure $S U(N)$ SUSY gauge theory. This must be added to the DV term in order to obtain the full effective superpotential. The total effective superpotential thus reads:

$$
W_{\text {eff }}=W_{V Y}+W_{D V}=N S\left(1-\log \frac{S}{\tilde{\Lambda}^{3}}\right)+N \frac{\partial}{\partial S} \mathcal{F}(S, g, m) .
$$

The holomorphic scale $\tilde{\Lambda}$ appearing in (125) is the one for the low-energy pure $S U(N)$ theory, when the massive matter field $\Phi$ has already been integrated out. Using the matching of scales (65), we see that it is related to the high energy scale $\Lambda$ by $\tilde{\Lambda}^{3}=m \Lambda^{2}$. Were we to be strict and use only the high energy scale in the VY superpotential, the matching of scales would come about from the vacuum diagram of the free matrix model, which is given by $S^{2} \log m$.

It may sound a bit unpleasant that one must add the VY piece "by hand". However, a very interesting fact well emphasized already in the earlier literature [1, 27] is that the VY superpotential can be fully recovered in the matrix model if one takes into account the volume of the $U(\hat{N})$ group rotating the hermitian matrix $M$. Essentially, one can show that:

$$
\langle 1\rangle \propto(\operatorname{Vol}(U(\hat{N})))^{-1} \simeq e^{\frac{\hat{N}^{2}}{2} \log \hat{N}+\ldots},
$$

so that after substituting for $S$, the leading term looks like a contribution to the planar free energy, and then the typical $S \log S$ term also arises from $(124)^{21}$. Although this observation can be justified by string duality it is difficult to find a field theoretical argument for it. In the framework we have been working, we have consistently taken $\langle 1\rangle=1$ and added the VY piece by hand.

\footnotetext{
${ }^{21}$ After the first version of this review appeared, the matrix model prescription which yields exactly the VY superpotential was spelled out in [28].
} 


\section{The chiral ring}

One of the uses of the effective superpotential is that of determining the vacuum structure of the theory, namely the expectation values of certain gauge invariant operators. It thus comes natural to define an equivalence class of such operators identifying those that have the same vacuum expectation value in a SUSY vacuum. This leads to the concept of the chiral ring that we now turn to discuss.

What we have seen in Section 2 is that a chiral superfield is defined by $\bar{D}_{\dot{\alpha}} \Phi=0$ and its lowest component $\phi(x)$ is annihilated by $\overline{\mathcal{Q}}_{\dot{\alpha}}$ :

$$
\left[\overline{\mathcal{Q}}_{\dot{\alpha}}, \phi(x)\right]=0 .
$$

The same facts are true for the gluino $\lambda_{\alpha}(x)$ which is the lowest component of the chiral superfield $W_{\alpha}$, only now we must switch commutators and anticommutators to account for the fact that $\lambda_{\alpha}(x)$ is a fermion:

$$
\left\{\overline{\mathcal{Q}}_{\dot{\alpha}}, \lambda_{\alpha}(x)\right\}=0 .
$$

We call chiral operators those gauge invariant operators $\mathcal{O}(x)$ that are annihilated by the $\overline{\mathcal{Q}}_{\dot{\alpha}}$. For, say, a bosonic operator:

$$
\left[\overline{\mathcal{Q}}_{\dot{\alpha}}, \mathcal{O}(x)\right]=0 .
$$

As we have seen before, given such an operator one can always generate its superpartners by acting on it with the $\mathcal{Q}_{\alpha}$ to fill up a chiral multiplet. Vice-versa, given a gauge invariant chiral superfield its lowest component will be a chiral operator.

Before going on studying the properties of such operators, we should discuss how to construct them in a gauge theory. Here we have at our disposal the gauge variant objects $\phi(x)$ and $\lambda_{\alpha}(x)$ that are annihilated by $\overline{\mathcal{Q}}_{\dot{\alpha}}$. One could thus construct gauge invariant composite operators, such as $\operatorname{tr} \lambda^{2}(x)$, that are naively annihilated by $\overline{\mathcal{Q}}_{\dot{\alpha}}$ by using the Leibniz rule. But one must be careful when defining composite operators in quantum field theory. In particular, when using point-splitting regularization, one must introduce a Wilson line to preserve gauge invariance, as we will discuss shortly. One then has to check that such regularization does not spoil eq. (129). In this case it turns out that the naive expectation is correct.

Consider, for concreteness, two fields $q$ and $\tilde{q}$, the lowest components of the quark chiral superfields in SQCD, transforming in the fundamental 
and antifundamental representations of the gauge group. Define the gauge invariant composite operator

$$
\begin{aligned}
\tilde{q} q(x) & =\lim _{\epsilon \rightarrow 0} \tilde{q}(x+\epsilon / 2) \mathrm{P} \exp \left(-i \int_{x-\epsilon / 2}^{x+\epsilon / 2} A_{\mu}(x) d x^{\mu}\right) q(x-\epsilon / 2) \\
& =\lim _{\epsilon \rightarrow 0} \tilde{q}(x+\epsilon / 2)\left(\mathbf{1}-i \epsilon^{\mu} A_{\mu}(x)+\ldots\right) q(x-\epsilon / 2),
\end{aligned}
$$

where the explicit insertion of the Wilson line guarantees gauge invariance. The dots in the second expression represent the high order terms in the Wilson line needed to preserve gauge invariance to all orders but they will not be needed here.

When acting with $\overline{\mathcal{Q}}_{\dot{\alpha}}$ on the LHS of (130) we get zero by definition when hitting $\tilde{q}$ and $q$ but the SUSY variation of $A_{\mu}$ gives rise to a gluino (cfr. eqs. (41) and (12):

$$
\left[\overline{\mathcal{Q}}_{\dot{\alpha}}, \tilde{q} q(x)\right]=-\frac{1}{\sqrt{2}} \lim _{\epsilon \rightarrow 0} \tilde{q}(x+\epsilon / 2) \epsilon_{\mu} \sigma_{\alpha \dot{\alpha}}^{\mu} \lambda^{\alpha}(x) q(x-\epsilon / 2) .
$$

The quantity in the limit is naively linear in $\epsilon$ but we could get into trouble if the OPE between $\tilde{q}$ and $q$ had a singularity in $\epsilon$ so that a non-zero result could arise. Fortunately, no such singularity is present in this case, as can be seen by noticing, for instance, that the propagator between $\tilde{q}$ and $q$ is zero (there is a non-zero propagator between $\tilde{q}$ and $\tilde{q}^{\dagger}$ and between $q$ and $\left.q^{\dagger}\right)$.

It is a general fact that there are no singularities in the OPE of lowest components of chiral fields and thus the gauge invariant composite operators made out of them obey the naive equation $\left[\overline{\mathcal{Q}}_{\dot{\alpha}}, \mathcal{O}(x)\right]=0$ and are thus chiral operators. We will see later, when discussing the Konishi anomaly, that there are other cases (not just involving $\phi(x)$ and $\lambda_{\alpha}(x)$ ) where such singularity arises and the naive SUSY transformations are modified.

Now that we have constructed chiral operators, we can immediately derive the important consequence [29] that the v.e.v. of an arbitrary (time ordered) product of such operators is totally independent of their spacetime position. Consider for instance the product of two bosonic chiral operators $\mathcal{O}_{1}(x)$ and $\mathcal{O}_{2}(y)$ and take the derivative with respect to $x^{\mu}$ :

$$
\begin{aligned}
& \frac{\partial}{\partial x^{\mu}}\left\langle 0\left|T\left(\mathcal{O}_{1}(x) \mathcal{O}_{2}(y)\right)\right| 0\right\rangle= \\
& \left\langle 0\left|T\left(\frac{\partial}{\partial x^{\mu}} \mathcal{O}_{1}(x) \mathcal{O}_{2}(y)\right)\right| 0\right\rangle+\delta_{\mu}^{0}\left\langle 0\left|\left[\mathcal{O}_{1}(x), \mathcal{O}_{2}(y)\right]\right| 0\right\rangle \delta\left(x^{0}-y^{0}\right)
\end{aligned}
$$


Both terms vanish separately. The first because (for, say, $x^{0}>y^{0}$ ):

$$
\begin{aligned}
\left\langle 0\left|\frac{\partial}{\partial x^{\mu}} \mathcal{O}_{1}(x) \mathcal{O}_{2}(y)\right| 0\right\rangle & =-i\left\langle 0\left|\left[\mathcal{P}_{\mu}, \mathcal{O}_{1}(x)\right] \mathcal{O}_{2}(y)\right| 0\right\rangle \\
& =\frac{i}{2} \bar{\sigma}_{\mu}^{\dot{\alpha} \alpha}\left\langle 0\left|\left[\left\{\mathcal{Q}_{\alpha}, \overline{\mathcal{Q}}_{\dot{\alpha}}\right\}, \mathcal{O}_{1}(x)\right] \mathcal{O}_{2}(y)\right| 0\right\rangle \\
& =\frac{i}{2} \bar{\sigma}_{\mu}^{\dot{\alpha} \alpha}\left\langle 0\left|\left\{\overline{\mathcal{Q}}_{\dot{\alpha}},\left[\mathcal{Q}_{\alpha}, \mathcal{O}_{1}(x)\right]\right\} \mathcal{O}_{2}(y)\right| 0\right\rangle \\
& =\frac{i}{2} \bar{\sigma}_{\mu}^{\dot{\alpha} \alpha}\left\langle 0\left|\left\{\overline{\mathcal{Q}}_{\dot{\alpha}},\left[\mathcal{Q}_{\alpha}, \mathcal{O}_{1}(x)\right] \mathcal{O}_{2}(y)\right\}\right| 0\right\rangle \\
& =0 .
\end{aligned}
$$

The idea behind the manipulations above is to use the SUSY algebra, the Jacobi identity and the chirality of the operators to bring $\overline{\mathcal{Q}}_{\dot{\alpha}}$ to act on the vacuum state, where it vanishes assuming that the vacuum is supersymmetric.

The second term in (132) is also zero because the equal time commutator vanishes since the same arguments leading to (133) apply more generally to the OPE of the two operators.

Given that the v.e.v. of chiral operators is independent of their position, one can go to the limit of large separation and apply cluster decomposition to obtain:

$$
\left\langle 0\left|T\left(\mathcal{O}_{1}\left(x_{1}\right) \ldots \mathcal{O}_{n}\left(x_{n}\right)\right)\right| 0\right\rangle=\left\langle\mathcal{O}_{1}\right\rangle \ldots\left\langle\mathcal{O}_{n}\right\rangle
$$

where there is no longer any need to specify the positions. This is the important property of factorization of correlation functions involving chiral operators.

Since objects of the type $\left\{\overline{\mathcal{Q}}_{\dot{\alpha}}, \ldots\right\}$ do not contribute to the expectation values in a SUSY vacuum, it is natural to define an equivalence relation between chiral operators. Two chiral operators $\mathcal{O}_{1}(x)$ and $\mathcal{O}_{2}(x)$ are equivalent if there exist a gauge invariant operator $X_{\dot{\alpha}}(x)$ such that

$$
\mathcal{O}_{1}(x)=\mathcal{O}_{2}(x)+\left\{\overline{\mathcal{Q}}^{\dot{\alpha}}, X_{\dot{\alpha}}(x)\right\}
$$

where the generalization to operators carrying Lorentz indices should be obvious - the extra indices just appear in the same way in $\mathcal{O}_{1}(x), \mathcal{O}_{2}(x)$ and $X_{\dot{\alpha}}(x)$ and the anticommutator in (135) becomes a commutator if $\mathcal{O}_{1}(x)$ and $\mathcal{O}_{2}(x)$ are fermionic.

Note that for (135) to be consistent, $X_{\dot{\alpha}}(x)$ must satisfy:

$$
\left[\overline{\mathcal{Q}}_{\dot{\beta}},\left\{\overline{\mathcal{Q}}^{\dot{\alpha}}, X_{\dot{\alpha}}(x)\right\}\right]=0
$$


which is not automatic from the SUSY algebra.

The set of equivalence classes of chiral operators under (135) forms a ring, known as the chiral ring. It is easy to check that the product of equivalence classes is well defined (i.e. it is independent of the representative) by applying the Leibniz rule a few times.

The equivalence (135) can also be formulated in superspace by saying that, if $\mathcal{O}_{1}(x)$ and $\mathcal{O}_{2}(x)$ are the lowest components of a chiral superfield $\Sigma_{1}$ and $\Sigma_{2}$ respectively, there exist a (gauge invariant) superfield $Y_{\dot{\alpha}}$ such that:

$$
\Sigma_{1}=\Sigma_{2}+\bar{D}^{\dot{\alpha}} Y_{\dot{\alpha}}
$$

We will also refer to two such superfields as equivalent in the chiral ring. Again, the superfield $Y_{\dot{\alpha}}$ must obey $\bar{D}_{\dot{\beta}} \bar{D}^{\dot{\alpha}} Y_{\dot{\alpha}}=0$ for consistency. Furthermore, the relation $\bar{D}_{\dot{\alpha}} Y_{\dot{\beta}}+\bar{D}_{\dot{\beta}} Y_{\dot{\alpha}}=0$ can always be imposed since it disappears from (137). These two conditions together allow one to "solve" the constraint as $Y_{\dot{\alpha}}=\bar{D}_{\dot{\alpha}} Z$, for some superfield $Z$ in terms of which (137) now reads

$$
\Sigma_{1}=\Sigma_{2}+\bar{D}^{\dot{\alpha}} \bar{D}_{\dot{\alpha}} Z=\Sigma_{2}+2 \bar{D}^{2} Z .
$$

Thus, two superfields are equivalent if they differ by a "chirally exact" term. We have also shown, in passing, that the lowest component of a chirally exact object is the SUSY variation of some other operator and, thus, its v.e.v. is identically zero in a SUSY vacuum.

Let us make all of these statements concrete and apply the above formalism to gauge theories. Let $\Phi$ be any chiral superfield transforming under the representation $T^{a}$ of the gauge group. We write $W_{\alpha}=W_{\alpha}^{a} T^{a}$. As usual, we denote the lowest components of $\Phi$ and $W_{\alpha}$ (in the WZ gauge) by $\phi$ and $-\sqrt{2} i \lambda_{\alpha}$ respectively. The basic relation that we will extensively use is:

$$
\lambda_{\alpha} \phi=-\frac{1}{2 \sqrt{2}}\left[\overline{\mathcal{Q}}^{\dot{\alpha}}, \nabla_{\alpha \dot{\alpha}} \phi\right]
$$

or, equivalently, in terms of superfields

$$
W_{\alpha} \Phi=\bar{\nabla}^{2} \nabla_{\alpha} \Phi
$$

Since the fields appearing in the Lagrangian are gauge variant we must use covariant derivatives. Of course, when acting on gauge invariant combinations, $\bar{\nabla}^{2}$ becomes $\bar{D}^{2}$.

The proof of these relations is simple: e.g.

$$
W_{\alpha} \Phi=\frac{i}{2}\left[\bar{\nabla}^{\dot{\alpha}}, \nabla_{\alpha \dot{\alpha}}\right] \Phi=\frac{i}{2} \bar{\nabla}^{\dot{\alpha}} \nabla_{\alpha \dot{\alpha}} \Phi=\frac{1}{2} \bar{\nabla}^{\dot{\alpha}}\left\{\bar{\nabla}_{\dot{\alpha}}, \nabla_{\alpha}\right\} \Phi=\bar{\nabla}^{2} \nabla_{\alpha} \Phi .
$$


(In the case where $\Phi$ is in the Adjoint representation, it is customary to think of it as a matrix and write $\left[W_{\alpha}, \Phi\right]$ instead.)

We now use (140) to obtain relations in the chiral ring. We call these relations "classical", and similarly for the ring generated by them, since they receive quantum corrections as we will discuss later on.

Let us specialize, for concreteness, to pure gauge theories, where the only chiral superfield is $W_{\alpha}$ itself, transforming in the adjoint representation. Eq. (140) reads:

$$
\left\{W_{\alpha}, W_{\beta}\right\}=\bar{\nabla}^{2} \nabla_{\alpha} W_{\beta} .
$$

Note that the RHS of (142) is symmetric in $\alpha$ and $\beta$ because of the relation $\nabla^{\alpha} W_{\alpha}=\bar{\nabla}_{\dot{\alpha}} \bar{W}^{\dot{\alpha}}$, which can be easily checked using the definitions of $W_{\alpha}$ and $\bar{W}_{\dot{\alpha}}$.

Consider now taking the trace of a string of $W_{\alpha}$. Because of (142), anticommuting any two $W_{\alpha}$ will contribute only a trivial element in the chiral ring, e.g.:

$$
\operatorname{tr}\left(W_{\alpha} W_{\beta} W_{\gamma}\right)=-\operatorname{tr}\left(W_{\alpha} W_{\gamma} W_{\beta}\right)+\bar{D}^{2} \operatorname{tr}\left(W_{\alpha} \nabla_{\beta} W_{\gamma}\right) .
$$

Using again (142), we see that the only non-trivial piece of the trace is when all the indices are antisymmetrized, thus excluding all traces of three or more $W_{\alpha}$.

Hence, of these type of invariants, the only one that is non-trivial in the chiral ring is the glueball superfield introduced in (71):

$$
S=-\frac{1}{32 \pi^{2}} \operatorname{tr} W^{\alpha} W_{\alpha}
$$

Notice that, in spite of the fact that $S \propto \bar{D}^{2} \operatorname{tr}\left(W^{\alpha} e^{-V}\left(D_{\alpha} e^{V}\right)\right)$, the glueball superfield is not chirally exact because $\bar{D}_{\dot{\alpha}} \operatorname{tr}\left(W^{\alpha} e^{-V}\left(D_{\alpha} e^{V}\right)\right)$ is not gauge invariant.

Let us now be more exhaustive and discuss the full chiral ring of all pure gauge theories based on simple Lie groups.

For a pure $S U(N)$ gauge theory, eq. (144) is the end of the story because traces of $W_{\alpha}$ exhaust all the invariants one can construct. In fact, the only primitive invariants at one's disposal to make a singlet are $\delta_{j}^{i}, \epsilon_{i_{1}, \ldots i_{N}}$ and $\epsilon^{i_{1}, \ldots i_{N}}$, but $\epsilon_{j_{1}, \ldots j_{N}} \times \epsilon^{i_{1}, \ldots i_{N}}$ is proportional to products of $\delta_{j}^{i}$ and one is only left with traces.

For other gauge groups one has to be more careful. For instance, for the remaining classical groups one could use the extra primitive invariants ( $\delta^{i j}=\delta^{j i}$ for the orthogonal and $\eta^{i j}=-\eta^{j i}$ for symplectic groups) to raise one index of the $W_{\alpha}$ and then try to use one $\epsilon_{i_{1}, \ldots i_{N}}$ to make a singlet. But 
even this will not work: For $S O(2 k+1)$ the $\epsilon$-tensor has an odd number of indices whereas one needs an even one. For $S p(k) W_{\alpha}^{i j}$ is symmetric and thus vanishes when contracted with $\epsilon$ and, finally, for $S O(2 k)(k>2)$ the contraction of two $W_{1}$ or of two $W_{2}$ with $\epsilon$ vanishes because of their Grassmann nature.

Thus, for pure gauge theories based on classical groups the chiral ring is generated by the glueball superfield 30. This is conjectured to be true even for exceptional groups although the presence of further primitive invariants (e.g. $\sigma^{i j k}$ for $G_{2}$ ) makes this statement non-trivial. Amongst the exceptional groups, so far the conjecture has been proven only for $G_{2}$ 31].

Having established the generators of the chiral ring, we now proceed to see that it has a further structure. An important and non-trivial fact that one can show using group theory is that relation (142) also implies

$$
S^{h}=0 \quad \text { in the classical chiral ring. }
$$

The integer $h$ is the dual Coxeter number of the group, i.e. half of the index of the adjoint representation. Eq. (145) has been proven for the classical groups [30] and for $G_{2}$ [31] and it is believed to be true for the other exceptional groups as well. The list of dual Coxeter numbers is given by Table 5. We will not go through the proof of this fact in general but just

\begin{tabular}{|c|c|c|c|c|c|c|c|c|}
\hline$S U(k)$ & $S O(2 k)$ & $S p(k)$ & $S O(2 k+1)$ & $G_{2}$ & $F_{4}$ & $E_{6}$ & $E_{7}$ & $E_{8}$ \\
\hline$k$ & $2 k-2$ & $k+1$ & $2 k-1$ & 4 & 9 & 12 & 18 & 30 \\
\hline
\end{tabular}

Table 2: Different groups and their dual Coxeter numbers.

notice that for $S U(2)$ it can be easily obtained from the relation (valid for any four $S U(2)$ generators)

$$
\operatorname{tr}(A B C D)=\frac{1}{2}(\operatorname{tr}(A B) \operatorname{tr}(C D)+\operatorname{tr}(A D) \operatorname{tr}(B C)-\operatorname{tr}(A C) \operatorname{tr}(D B)) .
$$

Substituting $A=B=W_{1}$ and $C=D=W_{2}$ and using Fermi statistics one gets

$$
S^{2} \propto\left(\operatorname{tr}\left(W_{1} W_{2}\right)\right)^{2}=\operatorname{tr}\left(W_{1} W_{1} W_{2} W_{2}\right),
$$

the last term being zero in the classical chiral ring.

What we have obtained so far is very similar to what was obtained in the previous section where imposing the condition (81) on the background 
has set to zero precisely all the operators that now are shown to be trivial in the classical chiral ring.

However, if eq. (145) were correct at the full quantum level, it would imply that in a SUSY vacuum, $\left\langle S^{h}\right\rangle=0 .{ }^{22}$ However, that $\left\langle S^{h}\right\rangle \neq 0$ can be shown explicitly by doing a one instanton calculation [23].

We thus face two choices, either $\left\langle S^{h}\right\rangle \neq 0$ is a signal of the fact that the vacuum breaks SUSY or the relation $S^{h}=0$ is modified by quantum corrections. There is overwhelming evidence that the second option is the right one. In particular the Witten index [32] for pure gauge theories is never zero implying that SUSY cannot be broken even by non-perturbative effects.

The fact that the chiral ring relation $S^{h}=0$ is modified to $S^{h}=$ const. is actually not that surprising. Remember that quantum corrections cannot bring new operators into existence but can modify the relations between existing ones by the introduction of the holomorphic scale $\Lambda$. The classical relation should be recovered by letting $\Lambda \rightarrow 0$. Simple dimensional analysis fixes $S^{h} \propto \Lambda^{3 h}$ and the instanton computation reviewed in 23] (see also 24]) allows also the determination of the proportionality constant $C(G)$. We recognize in $3 h$ the coefficient $\beta$ of the one loop beta-function of pure SYM. From (64) we can see that $\left\langle S^{h}\right\rangle$ is thus proportional to the exponential of minus the one instanton action $8 \pi^{2} / g^{2}$. Hence eq. (145) is changed into

$$
S^{h}=C(G) \Lambda^{3 h} \quad \text { in the quantum chiral ring. }
$$

So far we considered pure gauge theories. As it is going to be important later, let us consider an example of gauge theory with matter, say, a $U(N)$ gauge theory with fields $\Phi$ in the adjoint and $Q, \tilde{Q}$ in the fundamental and anti-fundamental. Relation (140) implies that, as far as the classical chiral ring is concerned, the fields $W_{\alpha}$ and $\Phi$ commute inside various singlets and that $W_{\alpha} Q=\tilde{Q} W_{\alpha}=0$. Thus the generators of the chiral ring are of the following type [33, 8, 34]:

$$
\operatorname{tr} \Phi^{k}, \quad \operatorname{tr} W_{\alpha} \Phi^{k}, \quad \operatorname{tr} W^{\alpha} W_{\alpha} \Phi^{k}, \quad \tilde{Q} \Phi^{k} Q
$$

Classically, they are subjected to the relation analogous to (145) for $S$ and to the usual relations stemming from the possibility of rewriting higher traces as functions of the lower ones. For instance, $\operatorname{tr} \Phi^{N+1}$ can be expressed as a polynomial in $\operatorname{tr} \Phi, \ldots, \operatorname{tr} \Phi^{N}$.

When matter fields are present, there are also relations that use the dynamics of the theory and depend on the particular choice of superpotential.

\footnotetext{
${ }^{22}$ By factorization one would also have $\langle S\rangle=0$.
} 
Let $\Phi_{r}$ denote a generic field content, with $r$ labeling the various irreducible representations of the gauge group. Let $F_{r}\left(W_{\alpha}, \Phi\right)$ be a combination of the fields that also transforms in the representation $r$. (Since we are working in the chiral ring, two such expressions that differ by a chirally exact operator can be identified.) The classical equations of motion for the matter fields are

$$
\bar{\nabla}^{2}\left(\bar{\Phi} e^{V}\right)^{r}=-\frac{\partial W(\Phi)}{\partial \Phi_{r}}
$$

Contracting with $F_{r}$ to make a singlet this implies the classical relation

$$
\frac{\partial W(\Phi)}{\partial \Phi_{r}} F_{r}\left(W_{\alpha}, \Phi\right)=0 \quad \text { in the classical chiral ring. }
$$

By now, it should not be surprising that also this classical relation is modified quantum mechanically. This is due to the so called Konishi anomaly which we now turn to discuss.

\section{The Konishi anomaly}

The Konishi anomaly is the SUSY extension of the usual chiral anomaly and it proves extremely useful in solving for the F-terms of the theory.

In its original form 9], it is based on the observation that (contrary to what we saw for chiral operators) the SUSY transformations of a generic composite operator are not always the ones that we would naively obtain applying the transformation to each component field. Consider again the example of SQCD, with its two chiral superfields $Q$ and $\tilde{Q}$ transforming in the fundamental and antifundamental representation of the gauge group. We saw in the previous section that the composite (chiral) operator $\tilde{q} q$ constructed from the lowest components obeys $\left[\overline{\mathcal{Q}}_{\dot{\alpha}}, \tilde{q} q\right]=0$ as one would naively expect.

If we drop the requirement that the composite operator be chiral, there are many other ways to build a gauge singlet even using just the components of one superfield instead of two. Of particular interest is the composite operator $\bar{\psi}^{\dot{\alpha}} q$ constructed only with the first and second components of $Q$. Naively, one would write:

$$
\begin{aligned}
\left\{\overline{\mathcal{Q}}_{\dot{\alpha}}, \bar{\psi}^{\dot{\alpha}} q\right\} & =\left\{\overline{\mathcal{Q}}_{\dot{\alpha}}, \bar{\psi}^{\dot{\alpha}}\right\} q-\bar{\psi}^{\dot{\alpha}}\left[\overline{\mathcal{Q}}_{\dot{\alpha}}, q\right] \\
& =2 \bar{f} q+0=-2 \frac{\partial W}{\partial q} q \quad \text { classical }
\end{aligned}
$$


If (152) was true, the RHS could not get a v.e.v. in a SUSY vacuum. For instance, for $W=m \tilde{Q} Q$ one would deduce that $\langle\tilde{q} q\rangle=0$, contradicting many well established facts about SQCD.

To obtain the right formula, we must once again regularize:

$$
\bar{\psi}^{\dot{\alpha}} q(x)=\lim _{\epsilon \rightarrow 0} \bar{\psi}^{\dot{\alpha}}(x+\epsilon / 2)\left(1-i \epsilon^{\mu} A_{\mu}(x)+\ldots\right) q(x-\epsilon / 2) .
$$

Now in the SUSY variation of $\bar{\psi}^{\dot{\alpha}} q$ there will be an extra piece coming from the SUSY variation of $A_{\mu}$ :

$$
\left\{\overline{\mathcal{Q}}_{\dot{\alpha}}, \bar{\psi}^{\dot{\alpha}} q\right\}=-2 \frac{\partial W}{\partial q} q-\frac{1}{\sqrt{2}} \lim _{\epsilon \rightarrow 0} \bar{\psi}_{\dot{\alpha}}(x+\epsilon / 2) \epsilon^{\mu} \bar{\sigma}_{\mu}^{\dot{\alpha} \alpha} \lambda_{\alpha}(x) q(x-\epsilon / 2)
$$

For our purposes it is enough to think of the gluino $\lambda$ as a background field, although this is not necessary. The extra piece seems to vanish as $\epsilon \rightarrow 0$ but now we have a singularity in the OPE coming from the presence of a term of the type $\sqrt{2} \int d^{4} x \bar{q} \lambda \psi$ in the Lagrangian. If one "brings down" such an interaction in the path integral one obtains the contribution (written for simplicity in the Abelian case)

$$
\begin{aligned}
& \lim _{\epsilon \rightarrow 0} \epsilon^{\mu} \bar{\sigma}_{\mu}^{\dot{\alpha} \alpha} \lambda_{\alpha}(x) \int \mathrm{d}^{4} x^{\prime}\left\langle 0\left|T\left(\psi_{\beta}\left(x^{\prime}\right) \bar{\psi}_{\dot{\alpha}}(x+\epsilon / 2)\right)\right| 0\right\rangle \lambda^{\beta}\left(x^{\prime}\right)\left\langle 0\left|T\left(q(x-\epsilon / 2) \bar{q}\left(x^{\prime}\right)\right)\right| 0\right\rangle \\
& \propto \lim _{\epsilon \rightarrow 0} \epsilon^{\mu} \bar{\sigma}_{\mu}^{\dot{\alpha} \alpha} \lambda_{\alpha}(x) \epsilon^{4} \frac{\epsilon_{\nu}}{\epsilon^{4}} \sigma_{\beta \dot{\alpha}}^{\nu} \lambda^{\beta}(x) \frac{1}{\epsilon^{2}} \propto \lambda^{2}(x) .
\end{aligned}
$$

More precisely, keeping track of all numerical factors and generalizing to the non-Abelian case one gets 9 ]

$$
\left\{\overline{\mathcal{Q}}_{\dot{\alpha}}, \bar{\psi}^{\dot{\alpha}} q\right\}=2\left(\bar{f} q+\frac{1}{16 \pi^{2}} \operatorname{tr} \lambda^{\alpha} \lambda_{\alpha}\right)
$$

As usual, the same relation can be expressed in terms of superfields as

$$
\bar{D}^{2}\left(\bar{Q} e^{V} Q\right)=-\frac{\partial W}{\partial Q} Q-\frac{1}{32 \pi^{2}} \operatorname{tr} W^{\alpha} W_{\alpha}
$$

This is so because we require the composite object $\bar{Q} e^{V} Q$ to be a superfield, i.e. to contain a supermultiplet of fields properly transforming into each other by a SUSY transformation. For instance:

$$
\bar{q} q \stackrel{\overline{\mathcal{Q}}}{\rightarrow} \bar{\psi}^{\dot{\alpha}} q \stackrel{\overline{\mathcal{Q}}}{\rightarrow} \bar{f} q+\frac{1}{16 \pi^{2}} \operatorname{tr} \lambda^{\alpha} \lambda_{\alpha}
$$

where the rightmost entry is thus the lowest component of $\bar{D}^{2} \bar{Q} e^{V} Q$. 
In the application of the Konishi anomaly to the recent developments, it is sometimes necessary to consider a generalization of formulas (156) and (157) 8 that we now turn to discuss. Consider chiral matter $\Phi$ in an arbitrary representation of the gauge group. Eq. (157) can be understood as the anomalous Schwinger-Dyson equation coming from varying $\Phi \rightarrow \Phi+\eta \Phi$ $(\eta \ll 1)$ in the path integral. The classical piece comes from the variation of the classical action whereas the anomaly comes from the non-invariance of the functional measure.

One can generalize the transformation to

$$
\Phi \rightarrow \Phi+\eta F\left(\Phi, W_{\alpha}\right)
$$

where $F$ is a holomorphic function transforming in the same representation as $\Phi$. The classical piece is straightforward:

$$
\bar{D}^{2}\left(\bar{\Phi} e^{V} F\right)=-\frac{\partial W}{\partial \Phi} F \quad \text { classical. }
$$

As for the anomalous piece, the only additional complication is in keeping track of the color indices. Let $r, s, \ldots$ denote the indices of the representation in which $\Phi$ transforms and let us write the gluino field as a matrix in that representation. The anomaly is, schematically ${ }^{23}$ :

$$
\lim \epsilon \bar{\psi}^{r} \lambda_{r}^{s} F_{s} \propto \lim \epsilon \int\left\langle\bar{\psi}^{r} \psi_{p}\right\rangle \lambda_{r}^{s} \lambda_{q}^{p}\left\langle F_{s} \bar{\phi}^{q}\right\rangle,
$$

where the spacetime dependence is as in (155). Using

$$
\left\langle\bar{\psi}^{r} \psi_{p}\right\rangle \propto \delta_{p}^{r} \quad \text { and } \quad\left\langle F_{s} \bar{\phi}^{q}\right\rangle \propto \frac{\partial F_{s}}{\partial \phi_{l}}\left\langle\phi_{l} \bar{\phi}^{q}\right\rangle \propto \frac{\partial F_{s}}{\partial \phi_{l}} \delta_{l}^{q}
$$

yields

$$
\lim \epsilon \bar{\psi}^{r} \lambda_{r}^{s} F_{s} \propto \lambda_{r}^{s} \lambda_{q}^{r} \frac{\partial F_{s}}{\partial \phi_{q}} .
$$

The generalized Konishi anomaly is thus:

$$
\bar{D}^{2}\left(\bar{\Phi}^{q}\left(e^{V}\right)_{q}^{r} F_{r}\right)=-\frac{\partial W}{\partial \Phi_{r}} F_{r}-\frac{1}{32 \pi^{2}} W_{r}^{\alpha s} W_{\alpha q}^{r} \frac{\partial F_{s}}{\partial \Phi_{q}} .
$$

\footnotetext{
${ }^{23}$ There can also be corrections from chirally exact terms that would contribute to the Dterms, but the non-renormalization theorem prevents additional perturbative contribution to the F-terms.
} 
The use of (164) is mainly in the context of the chiral ring, where we may set all chirally exact terms to zero, including the LHS of (164). In this context (164) reads:

$$
\frac{\partial W}{\partial \Phi_{r}} F_{r}=-\frac{1}{32 \pi^{2}} W_{r}^{\alpha s} W_{\alpha q}^{r} \frac{\partial F_{s}}{\partial \Phi_{q}} . \quad \text { in the quantum chiral ring }
$$

In particular, these relations hold for v.e.v.s in a SUSY vacuum, and they are often enough to solve for the F-terms of the effective theory. Specifically, they can be used to prove the Dijkgraaf - Vafa conjecture as we now discuss.

\section{Glueball superpotential via the Konishi anomaly}

In this section, we will exploit a relationship which exists between the effective superpotential and the SUSY vacuum expectation values of chiral operators to determine completely the former. The relation was discussed in Section 3 and reads:

$$
\frac{\partial}{\partial \lambda_{k}} W_{\text {eff }}=\left\langle X_{k}\right\rangle
$$

This relation was derived when we realized that integrating out and back in degrees of freedom was technically a Legendre transform. Alternatively, one can derive the above expression directly by expressing $W_{\text {eff }}$ in terms of the path integral over the elementary fields, weighted by the action including the tree level superpotential $W_{\text {tree }}=\sum \lambda_{k} X_{k}$. Then considering the coupling $\lambda_{k}$ as a source term for the operator $X_{k}$ the relation (166) follows.

The usefulness of eq. (166) is that if we are able to determine $\left\langle X_{k}\right\rangle$ as a function of the couplings $\lambda_{k}$ and of $S$ we can then obtain $W_{\text {eff }}$ up to a function independent of the coupling. The Konishi anomaly and its generalizations provide us with just a way to determine such expectation values. ${ }^{24}$

Let us see how this works in a simple example, where the non-generalized Konishi anomaly is sufficient to solve for a non-trivial $W_{\text {eff }}$ 36]. We take SQCD with gauge group $U(N)$ and, for simplicity, only one flavor ${ }^{25}$.

The only gauge invariant we can build with this matter content is a single meson superfield, $M=\tilde{Q}_{a} Q^{a}$. A tree level superpotential that one can write is the following:

$$
W_{\text {tree }}=m M+\lambda M^{2} .
$$

\footnotetext{
${ }^{24}$ That the Konishi anomaly plays an important role in the Dijkgraaf - Vafa conjecture was first emphasized in 35.

${ }^{25}$ The $U(1)$ factor is unimportant as it decouples in the IR.
} 
The quarks are thus massive, and the second term introduces a non-trivial interaction. The latter is non-renormalizable, but it is straightforward to see that (167) can be obtained by writing the renormalizable superpotential:

$$
W_{\text {tree }}=m \tilde{Q} Q+\frac{\mu}{2} \operatorname{tr} \Phi^{2}+g \tilde{Q} \Phi Q
$$

and then integrating out the adjoint field $\Phi$, assuming that $|\mu| \gg|m|$ and identifying $\lambda=-\frac{g^{2}}{2 \mu}$.

Let us now write down the Konishi anomaly relation for, say, the $U(1)_{Q}$ rotating the superfield $Q$. This is basically the computation leading to (157). Using (167), the relation in a SUSY vacuum becomes:

$$
m\langle M\rangle+2 \lambda\left\langle M^{2}\right\rangle=S .
$$

By using factorization, we can reexpress $\left\langle M^{2}\right\rangle=\langle M\rangle^{2}$ and then solve (169) for $\langle M\rangle$ :

$$
\langle M\rangle=-\frac{m}{4 \lambda} \pm \sqrt{\frac{m^{2}}{16 \lambda^{2}}+\frac{S}{2 \lambda}} .
$$

The two signs in (170) have a clear meaning in the classical limit, where $S \rightarrow 0$. For the positive sign, the v.e.v. tends to zero, and we are thus in a situation where classically the $U(N)$ gauge group in unbroken. For the negative sign, the v.e.v. tends to a finite value, and thus $U(N)$ is broken to $U(N-1)$. Note that when the coupling $\lambda$ is sent to zero this second classical vacuum is sent to infinity and disappears.

We thus have two partial differential equations for $W_{\text {eff }}$ :

$$
\frac{\partial W_{\mathrm{eff}}}{\partial m}=\langle M(S, m, \lambda)\rangle, \quad \frac{\partial W_{\mathrm{eff}}}{\partial \lambda}=\langle M(S, m, \lambda)\rangle^{2} .
$$

Using the expression (170), we obtain the solution:

$W_{\text {eff }}=-\frac{m^{2}}{8 \lambda} \pm \frac{m^{2}}{8 \lambda} \sqrt{1+\frac{8 \lambda}{m^{2}} S}+S \log \frac{m}{\Lambda}+S \log \left(1 \pm \sqrt{1+\frac{8 \lambda}{m^{2}} S}\right)+C(S)$,

where of course the coupling independent piece of $W_{\text {eff }}$ is still undetermined, and we have used the holomorphic scale $\Lambda$ to compensate for the dimension of $m$. This can be done because a rescaling in $\Lambda$ can always be absorbed in $C(S)$.

The coupling independent piece $C(S)$ can be determined by using lowenergy information on the theory. Consider simply the positive branch of (170), and take the coupling $\lambda$ to zero. Here we should recover the low energy 
physics of SQCD with a massive flavor. We know what this is, namely pure $S U(N)$ SYM, and we also know it is described by the VenezianoYankielowicz superpotential (75). By thus imposing that (172) with the positive signs reduces to (75) when $\lambda \rightarrow 0$ (for this, we have to match the holomorphic scale $\tilde{\Lambda}$ of the pure SYM theory to the scale $\Lambda$ of the theory with one flavor, $\tilde{\Lambda}^{3 N}=m \Lambda^{3 N-1}$ ) we obtain:

$$
\begin{aligned}
W_{\mathrm{eff}}= & N S\left(1-\log \frac{S}{\Lambda^{3}}\right)+S \log \frac{m}{\Lambda}-\frac{S}{2} \\
& -\frac{m^{2}}{8 \lambda} \pm \frac{m^{2}}{8 \lambda} \sqrt{1+\frac{8 \lambda}{m^{2}} S}+S \log \left(\frac{1}{2} \pm \frac{1}{2} \sqrt{1+\frac{8 \lambda}{m^{2}} S}\right) .
\end{aligned}
$$

We have thus determined the full effective superpotential by using the Konishi anomaly (169) to determine the v.e.v.s, which have allowed us to solve for the coupling dependence of $W_{\text {eff }}$. The remaining piece is then determined by taking specific values of the coupling constants which make the low-energy physics more familiar.

It is worth noting that the same result (173) can be obtained through a matrix-vector model based on the tree level superpotential (168) 37. There one can show that only the graphs with the topology of a disk give a non-trivial contribution, the boundary of the disk being composed of quark propagators. The result of this perturbative computation is a power series in $S$, which sums to the exact expression (173). In this latter approach the VY piece of $W_{\text {eff }}$ has to be added to the result.

We now turn to the example discussed in [8], where the generalized Konishi anomaly relations will be needed. We consider a $U(N)$ gauge theory with a matter field in the adjoint representation and a generic tree level superpotential:

$$
W_{\text {tree }}=\sum_{k=0}^{n} \frac{g_{k}}{k+1} \operatorname{tr} \Phi^{k+1} .
$$

The classical vacuum structure is determined by the extrema of $W_{\text {tree }}(z)$, seen as a holomorphic function of a complex variable. If we assume that the $n$ extrema are all distinct:

$$
W_{\text {tree }}^{\prime}(z)=g_{n} \prod_{i=1}^{n}\left(z-a_{i}\right), \quad a_{i} \neq a_{j} \quad \text { for } \quad i \neq j
$$

then in the classical vacuum the eigenvalues of $\Phi$ will partition into groups corresponding to every extremum and the fluctuations around these vacua 
will all be massive. If we have $N_{i}$ eigenvalues equal to $a_{i}$, the classical gauge symmetry is spontaneously broken in the following way:

$$
U(N) \rightarrow \prod_{i=1}^{n} U\left(N_{i}\right) .
$$

At the quantum level, we expect for every $U\left(N_{i}\right)$ factor that at low enough energies the $S U\left(N_{i}\right)$ piece will confine, while the $U(1)$ piece will be in a free Coulomb phase. For every factor, we thus define the effective fields:

$$
\begin{aligned}
S_{i} & =-\frac{1}{32 \pi^{2}} \operatorname{tr} W_{i}^{\alpha} W_{\alpha i}, \\
w_{i}^{\alpha} & =\frac{1}{4 \pi} \operatorname{tr} W_{i}^{\alpha} .
\end{aligned}
$$

The quantity that we want to determine is $W_{\text {eff }}$ as a function of the couplings $g_{k}$ and the effective fields $S_{i}$ and $w_{i}^{\alpha}$. Notice that the trace in (177) is over the full $U\left(N_{i}\right)$ subgroup and not $S U\left(N_{i}\right)$ for later convenience.

We now make a crucial remark that simplifies the discussion. The overall $U(1)$ of the full $U(N)$ gauge group is free, since the matter field is in the adjoint representation. This also means that there are no Yukawa couplings involving the gaugino relative to this $U(1)$, and thus its action is also one of a free fermion. We thus have the possibility of shifting the $U(1)$ gaugino by a constant Weyl spinor without changing anything in the theory. In superfield terms, this symmetry of the theory is implemented as:

$$
W_{\alpha} \rightarrow W_{\alpha}-4 \pi \chi_{\alpha} \mathbf{1}_{N \times N}
$$

When the gauge symmetry is broken as in (176), the symmetry still acts as an overall simultaneous shift of all the gaugino superfields, $W_{\alpha i} \rightarrow W_{\alpha i}-$ $4 \pi \chi_{\alpha} \mathbf{1}_{N_{i} \times N_{i}}$, so that the effective fields transform as:

$$
\begin{aligned}
S_{i} & \rightarrow S_{i}+\chi^{\alpha} w_{\alpha i}-\frac{1}{2} N_{i} \chi^{\alpha} \chi_{\alpha}, \\
w_{i}^{\alpha} & \rightarrow w_{i}^{\alpha}-N_{i} \chi^{\alpha} .
\end{aligned}
$$

The effective superpotential $W_{\text {eff }}\left(S_{i}, w_{i}^{\alpha}, g_{k}\right)$ has to be invariant under the above transformation, since it cannot depend on the decoupled overall $U(1)$.

A clever way to constrain the form of $W_{\text {eff }}$ using the invariance under (180) is to apply to this fermionic symmetry the same tricks used with supersymmetry in Section 2. Namely, consider the invariance as a translation in an auxiliary Grassmann variable $\psi^{\alpha}$. Then build a "superfield" using 
$\psi^{\alpha}$ such that the translation $\psi^{\alpha} \rightarrow \psi^{\alpha}+\chi^{\alpha}$ implements (180). Finally, an invariant function is trivially built by writing the integral over $\psi$-superspace of any function of the $\psi$-superfield. Hence, we define:

$$
\begin{aligned}
\mathcal{S}_{i} & =-\frac{1}{2} \operatorname{tr}\left(\frac{W_{i}^{\alpha}}{4 \pi}-\psi^{\alpha}\right)\left(\frac{W_{\alpha i}}{4 \pi}-\psi_{\alpha}\right) \\
& =S_{i}+w_{i}^{\alpha} \psi_{\alpha}-\frac{1}{2} N_{i} \psi^{\alpha} \psi_{\alpha} .
\end{aligned}
$$

A manifestly invariant effective superpotential is then given by:

$$
W_{D V}=-\int \mathrm{d}^{2} \psi \mathcal{F}\left(\mathcal{S}_{i}, g_{k}\right),
$$

which, after performing the Grassmann integral, becomes:

$$
W_{D V}=\sum_{i} N_{i} \frac{\partial \mathcal{F}}{\partial S_{i}}+\frac{1}{2} \sum_{i, j} \frac{\partial^{2} \mathcal{F}}{\partial S_{i} \partial S_{j}} w_{i}^{\alpha} w_{\alpha j} .
$$

To compute the derivatives we can consider $\mathcal{F}$ as a function of the $S_{i}$, that is the lowest components of the $\mathcal{S}_{i}$.

Note that (183) is quadratic in $w_{i}^{\alpha}$, a result which could also be obtained in the perturbative set up of Section 4 by observing that only planar diagrams contribute. We should stress here that this feature appears only when $S_{i}$ is the glueball field obtained by tracing over $U\left(N_{i}\right)$ and not over its $S U\left(N_{i}\right)$ subgroup. If we were to consider the latter, then $W_{\text {eff }}$ would contain higher powers of $w_{i}^{\alpha}$.

Let us anticipate now that the main result of this section will be to identify the function $\mathcal{F}\left(S_{i}, g_{k}\right)$ with the planar free energy of the related matrix model, as eq. (124) at the end of Section 4 suggests. To see how this comes about, let us derive equations for this function $\mathcal{F}$.

First of all, the coupling dependence of the effective superpotential is fixed by the equations:

$$
\frac{\partial W_{\mathrm{eff}}}{\partial g_{k}}=\left\langle\frac{1}{k+1} \operatorname{tr} \Phi^{k+1}\right\rangle
$$

Using eq. (182), the above equation reads:

$$
\int d^{2} \psi \frac{\partial}{\partial g_{k}} \mathcal{F}\left(\mathcal{S}_{i}, g_{l}\right)=-\left\langle\frac{1}{k+1} \operatorname{tr} \Phi^{k+1}\right\rangle .
$$


We can write an equation directly for the integrand $\mathcal{F}$ if we write the RHS as the highest component of a $\psi$-superfield, generalizing (181). We thus have:

$$
\frac{\partial}{\partial g_{k}} \mathcal{F}\left(\mathcal{S}_{i}, g_{l}\right)=-\left\langle\frac{1}{2(k+1)} \operatorname{tr}\left(\frac{W^{\alpha}}{4 \pi}-\psi^{\alpha}\right)\left(\frac{W_{\alpha}}{4 \pi}-\psi_{\alpha}\right) \Phi^{k+1}\right\rangle .
$$

For convenience, the equations can be written setting $\psi^{\alpha}=0$ without loss of information, since from the lowest components the higher ones are generated acting as in (179). The equation we will thus take as defining the coupling dependent part of the function $\mathcal{F}$ is:

$$
\frac{\partial}{\partial g_{k}} \mathcal{F}\left(S_{i}, g_{l}\right)=-\left\langle\frac{1}{k+1} \operatorname{tr} \frac{W^{\alpha} W_{\alpha}}{32 \pi^{2}} \Phi^{k+1}\right\rangle .
$$

The next step is to introduce a generating function for the operators on the RHS of the above equation. It reads:

$$
R(z)=-\frac{1}{32 \pi^{2}}\left\langle\operatorname{tr} \frac{W^{\alpha} W_{\alpha}}{z-\Phi}\right\rangle \equiv-\frac{1}{32 \pi^{2}} \sum_{k=0}^{\infty} \frac{1}{z^{k+1}}\left\langle\operatorname{tr} W^{\alpha} W_{\alpha} \Phi^{k}\right\rangle .
$$

Note that in the above we are not really paying attention to the ordering of the $W^{\alpha}$ with respect to the $\Phi$, the reason being the relation in the chiral ring $\left[W^{\alpha}, \Phi\right]=0$.

The expression (188) also gives us a more precise, gauge invariant, definition of the gaugino condensates $S_{i}$. Indeed, suppose that the analytic structure of the function $R(z)$ is such that it has $n$ cuts over the $z$-plane (we will shortly see how this arises). The cuts can be seen as a sort of quantum resolution of simple poles. By defining the contour $C_{i}$ as the one going around the $i$-th cut, we define:

$$
S_{i}=\frac{1}{2 \pi i} \oint_{C_{i}} \mathrm{~d} z R(z) .
$$

Classically, the above relation is understood as follows. If the contour $C_{i}$ encircles the eigenvalue $a_{i}$, then for any matrix $M$ the integral projects on the eigenspace corresponding to this eigenvalue. Indeed, having diagonalized $\Phi$, we have:

$$
\begin{aligned}
\frac{1}{2 \pi i} \oint_{C_{i}} \mathrm{~d} z \operatorname{tr} \frac{M}{z-\Phi} & =\frac{1}{2 \pi i} \oint_{C_{i}} \mathrm{~d} z \sum_{m=1}^{N} \frac{M_{m m}}{z-\phi_{m}} \\
& =\sum_{\phi_{m}=a_{i}} M_{m m}=\operatorname{tr} P_{i} M \equiv \operatorname{tr} M_{i}
\end{aligned}
$$


Note that we also have $\operatorname{tr} P_{i}=N_{i}$. Since projectors should not receive quantum corrections, the result of the manipulation above carries over to the quantum level.

We are now after a way to solve for the generating function (also called resolvent, in reference to the matrix model analogy) $R(z)$. We are going to see that it is precisely the generalized Konishi anomaly which provides us with a closed equation for it.

Let us first of all specialize eq. (165) to the case of a matter field in the adjoint. The LHS simply becomes a trace. As for the RHS, we have to make explicit the adjoint action of the gaugino superfield by writing $W_{s}^{\alpha r} \equiv i W^{\alpha p} f_{s}^{p r}$, with all indices in the adjoint representation. Then the two structure constants are traded for two commutators, and the generalized Konishi anomaly reads:

$$
\left\langle\operatorname{tr}\left(W_{\text {tree }}^{\prime}(\Phi) F\left(W^{\alpha}, \Phi\right)\right)\right\rangle=-\frac{1}{32 \pi^{2}} \sum_{i, j=1}^{N}\left\langle\left\{W^{\alpha},\left[W_{\alpha}, \frac{\partial F}{\partial \Phi_{j}^{i}}\right]\right\}_{j}^{i}\right\rangle .
$$

We now specialize to a particular variation of $\Phi$, namely we take:

$$
\delta \Phi=-\eta \frac{1}{32 \pi^{2}} \frac{W^{\alpha} W_{\alpha}}{z-\Phi} .
$$

To see what the RHS of (191) looks like with this variation, we develop in a power series as in (188):

$$
\begin{aligned}
\frac{\partial}{\partial \Phi_{j}^{i}}\left(\frac{W^{\alpha} W_{\alpha}}{z-\Phi}\right)_{l}^{k} & =\sum_{n=0}^{\infty} \frac{1}{z^{n+1}} \frac{\partial}{\partial \Phi_{j}^{i}}\left(W^{\alpha} W_{\alpha} \Phi^{n}\right)_{l}^{k} \\
& =\sum_{n=1}^{\infty} \sum_{p=0}^{n-1} \frac{1}{z^{n+1}}\left(W^{\alpha} W_{\alpha} \Phi^{p}\right)_{i}^{k}\left(\Phi^{n-p-1}\right)_{l}^{j} \\
& =\sum_{p=0}^{\infty} \sum_{n=p+1}^{\infty} \frac{1}{z^{n+1}}\left(W^{\alpha} W_{\alpha} \Phi^{p}\right)_{i}^{k}\left(\Phi^{n-p-1}\right)_{l}^{j} \\
& =\sum_{p=0}^{\infty} \sum_{m=0}^{\infty} \frac{1}{z^{p+1} z^{m+1}}\left(W^{\alpha} W_{\alpha} \Phi^{p}\right)_{i}^{k}\left(\Phi^{m}\right)_{l}^{j} \\
& =\left(\frac{W^{\alpha} W_{\alpha}}{z-\Phi}\right)_{i}^{k}\left(\frac{1}{z-\Phi}\right)_{l}^{j} .
\end{aligned}
$$

Implementing now the chiral ring relations, which forbid traces with more than two $W^{\alpha}$, we see that only one term of the (anti)commutators in (191) 
is non-vanishing, namely when the four $W^{\alpha}$ distribute themselves by two in each trace. We thus get the following basic relation in a SUSY vacuum:

$$
-\frac{1}{32 \pi^{2}}\left\langle\operatorname{tr}\left(W_{\text {tree }}^{\prime}(\Phi) \frac{W^{\alpha} W_{\alpha}}{z-\Phi}\right)\right\rangle=\frac{1}{\left(32 \pi^{2}\right)^{2}}\left\langle\left(\operatorname{tr} \frac{W^{\alpha} W_{\alpha}}{z-\Phi}\right)^{2}\right\rangle .
$$

At this point, we can crucially use the factorization property of the correlation functions of chiral operators to write the RHS as $R(z)^{2}$. Concerning the LHS, we can add and subtract $W_{\text {tree }}^{\prime}(z)$ to $W_{\text {tree }}^{\prime}(\Phi)$ so that we can write:

$$
-\frac{1}{32 \pi^{2}}\left\langle\operatorname{tr}\left(W_{\text {tree }}^{\prime}(\Phi) \frac{W^{\alpha} W_{\alpha}}{z-\Phi}\right)\right\rangle=W_{\text {tree }}^{\prime}(z) R(z)+\frac{1}{4} f_{n-1}(z),
$$

where we have defined:

$$
f_{n-1}(z)=\frac{1}{8 \pi^{2}}\left\langle\operatorname{tr}\left(\left(W_{\text {tree }}^{\prime}(z)-W_{\text {tree }}^{\prime}(\Phi)\right) \frac{W^{\alpha} W_{\alpha}}{z-\Phi}\right)\right\rangle .
$$

It is easy to convince oneself that $f_{n-1}(z)$ is a polynomial of degree $n-1$ : $f_{n-1}(z) \equiv \sum_{k=0}^{n-1} f_{k} z^{k}$. Indeed, by expanding $W_{\text {tree }}^{\prime}(z)$ in the numerator around any eigenvalue of $\Phi$, one can see that $f_{n-1}(z)$ has no singularities. Moreover, we see that at large $z$, the LHS of (195) goes like $1 / z$. Thus $f_{n-1}(z)$ must cancel all the non-negative powers in the product $W_{\text {tree }}^{\prime}(z) R(z)$, which go at most as $z^{n-1}$. At this point the $n$ coefficients of $f_{n-1}(z)$ are unknown complex parameters, to be related to more physically significant quantities shortly.

We have thus finally obtained the equation for $R(z)$. It reads:

$$
R(z)^{2}=W_{\text {tree }}^{\prime}(z) R(z)+\frac{1}{4} f_{n-1}(z) .
$$

Solving the above quadratic equation yields

$$
R(z)=\frac{1}{2}\left(W_{\text {tree }}^{\prime}(z)-\sqrt{W_{\text {tree }}^{\prime}(z)^{2}+f_{n-1}(z)}\right),
$$

where the sign has been chosen in order to get the right $1 / z$ behavior at infinity. The above solution gives us an expression of $R(z)$ (and thus, expanding, of all the relevant v.e.v.s) in terms of the couplings $g_{k}$ and the coefficients $f_{k}$ of the polynomial.

In order to have a useful solution for $R(z)$, namely one which is a function of $g_{k}$ and the $S_{i}$, we only have to use the relations (189) to get expressions of the $S_{i}$ in terms of the $f_{k}$ and $g_{k}$. By inverting these relations, we finally 
obtain an expression $R(z)$ in terms of $g_{k}$ and $S_{i}$ that can be used to integrate for $\mathcal{F}\left(S_{i}, g_{k}\right)$. Fortunately, in this case we do not need to perform this computation explicitly because the implicit expression (189) is all we need to show the correspondence with the matrix model.

We now pause to mention what should be done in order to perform a more complete treatment of this theory. One defines two more generating functions:

$$
w_{\alpha}(z)=\frac{1}{4 \pi}\left\langle\operatorname{tr} \frac{W_{\alpha}}{z-\Phi}\right\rangle, \quad T(z)=\left\langle\operatorname{tr} \frac{1}{z-\Phi}\right\rangle,
$$

which can be seen as higher components of $R(z)$ in a $\psi$-superfield. Anomaly equations for them can also be written, which can be solved linearly. New polynomials with undetermined coefficients like $f_{n-1}(z)$ arise, but again these coefficients can be solved for the physical quantities:

$$
w_{\alpha}^{i}=\frac{1}{2 \pi i} \oint_{C_{i}} \mathrm{~d} z w_{\alpha}(z), \quad N_{i}=\frac{1}{2 \pi i} \oint_{C_{i}} \mathrm{~d} z T(z) .
$$

In principle, it is the expansion of $T(z)$ that is needed to obtain $W_{\text {eff }}$, but as we have seen, the $\psi$ translational symmetry makes it possible to consider only the resolvent $R(z)$ to fully solve the problem. This is no longer true for some theories with a different gauge group and/or matter content, for which one cannot write the fermionic $\psi$ symmetry. One can still write generalized Konishi anomaly relations that can be solved, but in this case one has eventually to get the expression of the analog of $T(z)$ in order to obtain $W_{\text {eff }}$.

What we are going to show in the following is that there is an even more direct way to compute $\mathcal{F}\left(S_{i}, g_{k}\right)$ through a bosonic matrix model computation. To prove this, we are going to show that exactly the same equations for $R(z)$ and $\mathcal{F}$ arise within the matrix model.

Let us define the free energy $\hat{\mathcal{F}}$ of the matrix model whose potential is given by $W_{\text {tree: }}$

$$
e^{-\frac{\hat{N}^{2}}{\hat{S}^{2}} \hat{\mathcal{F}}}=\int \mathrm{d} M e^{-\frac{\hat{N}}{\hat{S}} W_{\text {tree }}(M)},
$$

where $M$ are hermitian $\hat{N} \times \hat{N}$ matrices and we take $\hat{N}$ to be large. Note that $\hat{N}$ is not related to the $N$ of the gauge theory. For the moment we may also think $\hat{S}$ as unrelated to $S$ although a relation between these last quantities will arise in the following. 
First of all we derive differential equations for $\hat{\mathcal{F}}$ by taking derivatives with respect to the couplings $g_{k}$ on both sides:

$$
\frac{\partial \hat{\mathcal{F}}}{\partial g_{k}}=\frac{\hat{S}}{\hat{N}}\left\langle\frac{1}{k+1} \operatorname{tr} M^{k+1}\right\rangle .
$$

In the large $\hat{N}$ limit, both sides of the above equation will have a genus expansion, with the planar contribution being the leading one. (With this normalization they both tend to a finite limit.)

As in (188), we introduce a generating function for the v.e.v.s on the RHS of (202). This is called the matrix model resolvent:

$$
\hat{R}(z)=\frac{\hat{S}}{\hat{N}}\left\langle\operatorname{tr} \frac{1}{z-M}\right\rangle .
$$

We are now left with the task of deriving an equation for the resolvent. Similarly to the Konishi anomaly, we can obtain such an equation from the Ward identities implied by reparameterization invariance of the matrix model free energy. Namely, the integral in (201) should be invariant under a simultaneous reparameterization of the matrix $M \rightarrow M+\delta M$ both in the measure and in the potential. Let us take the following variation:

$$
\delta M=\eta \frac{1}{z-M}, \quad \eta \ll 1 .
$$

The measure changes as:

$$
d M \rightarrow d M\left(1+\frac{\partial \delta M_{i}^{j}}{\partial M_{i}^{j}}\right)
$$

Now, using the same trick as in (193), we have that:

$$
\frac{\partial}{\partial M_{i}^{j}}\left(\frac{1}{z-M}\right)_{i}^{j}=\left(\operatorname{tr} \frac{1}{z-M}\right)^{2} .
$$

On the other hand, the variation (204) acts on the potential by bringing down its derivative, so that the Ward identity reads:

$$
\frac{\hat{S}}{\hat{N}}\left\langle\left(\operatorname{tr} \frac{1}{z-M}\right)^{2}\right\rangle=\left\langle\operatorname{tr} \frac{W_{\text {tree }}^{\prime}(M)}{z-M}\right\rangle .
$$


This expression can be turned into an equation for $\hat{R}(z)$ again using factorization, but this time the reason we can factorize is very different from the one in the gauge theory context. Indeed, we can write:

$$
\frac{\hat{S}^{2}}{\hat{N}^{2}}\left\langle\left(\operatorname{tr} \frac{1}{z-M}\right)^{2}\right\rangle \rightarrow \frac{\hat{S}^{2}}{\hat{N}^{2}}\left(\left\langle\operatorname{tr} \frac{1}{z-M}\right\rangle\right)^{2}=\hat{R}(z)^{2},
$$

only in the planar limit, that is, keeping only the leading order in $\hat{N}$ for both expressions.

This can be understood as follows. Consider the correlation of two operators $(1 / \hat{N}) \operatorname{tr} M^{4}$ as depicted in Fig. 7. The diagrams contributing to
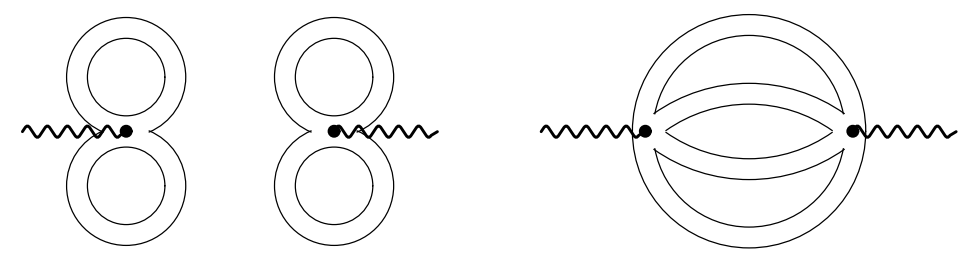

Figure 7: Factorization - the disconnected two-point function of the operator $(1 / \hat{N}) \operatorname{tr} M^{4}$ is finite while the connected one is suppressed by a factor $1 / \hat{N}^{2}$.

the correlator have the same form of vacuum diagrams, only this time at the point where the operator is inserted we have a factor $(1 / \hat{N})$ instead of $\hat{N}$ as there would be for an ordinary vertex. Thus we see that connected diagrams with one insertion are finite while those with two insertions are suppressed by $\left(1 / \hat{N}^{2}\right)$ leading to the abovementioned factorization property. The mechanism leading to factorization here is thus totally different from the gauge theory context, where supersymmetry and the properties of chiral operators are crucially used.

As for the RHS of (207), we apply the same addition and subtraction of $W_{\text {tree }}^{\prime}(z)$ to write the final equation for the resolvent, known as the loop equation, as:

$$
\hat{R}(z)^{2}=W_{\text {tree }}^{\prime}(z) \hat{R}(z)+\frac{1}{4} \hat{f}_{n-1}(z)
$$

with:

$$
\hat{f}_{n-1}(z)=4 \frac{\hat{S}}{\hat{N}}\left\langle\operatorname{tr} \frac{W_{\text {tree }}^{\prime}(M)-W_{\text {tree }}^{\prime}(z)}{z-M}\right\rangle,
$$

also an unknown polynomial of degree $n-1$. We thus see that the loop equation for $\hat{R}(z)$ is the same as eq. (197) for $R(z)$ and thus that $\hat{R}(z)$ 
has the same analytic structure as $R(z)$ in the gauge theory, namely it will display a total of $n$ cuts.

As before, the coefficients of the function $\hat{f}_{n-1}(z)$ can be reexpressed in terms of the following variables:

$$
\hat{S}_{i}=\frac{1}{2 \pi i} \oint_{C_{i}} \mathrm{~d} z \hat{R}(z)
$$

where the contour $C_{i}$ goes around the $i$-th cut $^{26}$. Using the definition (203) of the resolvent, and applying the same reasoning as in (190), we obtain:

$$
\hat{S}_{i}=\frac{1}{2 \pi i} \oint_{C_{i}} \mathrm{~d} z \frac{\hat{S}}{\hat{N}}\left\langle\operatorname{tr} \frac{1}{z-M}\right\rangle=\frac{\hat{S}}{\hat{N}} \hat{N}_{i} .
$$

The $\hat{S}_{i}$ are proportional to the fraction of eigenvalues populating the $i$-th cut $^{27}$.

We are now finally ready to state the correspondence between the matrix model and the gauge theory computation. We already have the same potential $W_{\text {tree }}(z)$ entering the equation for the resolvent. We now identify also the two polynomials of degree $n-1$, that is we impose $\hat{f}_{n-1}(z)=f_{n-1}(z)$ so that the coefficients entering the loop equations are all the same. We can then trivially identify the two resolvents: $\hat{R}(z)=R(z)$. This in turn implies $\hat{S}_{i}=S_{i}$. Lastly, expanding the resolvents we also obtain identical equations for the free energy on one side and for the function $\mathcal{F}$ on the other, so that we identify $\hat{\mathcal{F}}\left(S_{i}, g_{k}\right)=\mathcal{F}\left(S_{i}, g_{k}\right)$.

This is thus the core of the correspondence between the computation of the effective superpotential on the gauge theory side, and the free energy of the matrix model. Using a mixed notation, and recalling the relation between $\mathcal{F}$ and $W_{D V}$, we have that:

$$
W_{D V}=\sum_{i} N_{i} \frac{\partial \hat{\mathcal{F}}}{\partial S_{i}}
$$

where we concentrate on the piece of $W_{D V}$ relevant purely to the glueball superfields.

There are some important remarks one has to make on (213). The first is to state once again that the $N_{i}$ are not related to the $\hat{N}_{i}$, the former being

\footnotetext{
${ }^{26}$ Note that in the hermitian matrix model context, the cuts are on the real axis and thus the $\hat{S}_{i}$ are real, unlike the gauge theory context where both can be generally complex. The relation between the quantities on the two sides will be done by analytic continuation.

${ }^{27}$ This last relation has no analogue in the gauge theory.
} 
finite and the latter being taken to infinity in order to extract the planar contribution to the free energy (and to be able to use the factorization in (208)).

The second remark is that once the correspondence is derived as before, one can turn to any other alternative derivation of $\hat{\mathcal{F}}$ on the matrix model side in order to compute it, as for instance presented in Appendix B. Then, when the result is stated in terms of the filling fractions $\hat{S}_{i}$ and the couplings $g_{k}$, the gauge theory effective superpotential is obtained using (213).

The last comment on (213) is that one could take it at face value and apply it even to any part of the matrix model free energy $\hat{\mathcal{F}}$ which depends only on the filling fractions $\hat{S}_{i}$ and not on the couplings $g_{k}$. That would imply the possibility of deriving on the gauge theory side the effective superpotential also for the pure gauge low-energy dynamics. Keeping in mind that all the arguments of this section do not apply to this case, one can still perform a simple computation and find, very similarly to (126), that the Veneziano-Yankielowicz superpotential is reproduced. This fact still needs a better understanding in gauge theoretic terms.

\section{Acknowledgments}

We thank Vanicson L. Campos for collaborating with us on work related to this review. One of us (G.F.) wishes to thank the organizers and the participants of the schools at Nordita and Parma in which part of the material in this review was tried out. R.A. would like to thank the ITP in Göteborg for support and warm hospitality during most of the work leading up to this review. We also thank Matteo Bertolini, Massimo Bianchi, Paolo di Vecchia, Alessandra Feo, Francesco Fucito, Kumar Narain, Kostas Sfetsos and Daniela Zanon for discussions.

This work is partly supported by EU contracts HPRN-CT-2000-00122 and HPRN-CT-00131, by the "Actions de Recherche Concertées" of the "Direction de la Recherche Scientifique - Communauté Française de Belgique", by a "Pôle d'Attraction Interuniversitaire" (Belgium) and by IISNBelgium (convention 4.4505.86). R.A. is a Postdoctoral Researcher of the Fonds National de la Recherche Scientifique (Belgium). The research of G.F. is supported by the Swedish Research Council (Vetenskapsrådet) contract 622-2003-1124. 


\section{A Superspace notation}

In this appendix we collect the notation and conventions used throughout this paper.

The Minkowski metric is $\eta^{\mu \nu}=\operatorname{diag}(+1,-1,-1,-1)$.

The gamma matrices $\gamma^{\mu}$ satisfy the Clifford algebra $\left\{\gamma^{\mu}, \gamma^{\nu}\right\}=2 \eta^{\mu \nu}$, and we always use the Weyl representation

$$
\gamma^{\mu}=\left(\begin{array}{cc}
0 & \sigma^{\mu} \\
\bar{\sigma}^{\mu} & 0
\end{array}\right)
$$

where

$$
\begin{array}{ll}
\sigma^{0}=\bar{\sigma}^{0}=\left(\begin{array}{cc}
-1 & 0 \\
0 & -1
\end{array}\right), & \sigma^{1}=-\bar{\sigma}^{1}=\left(\begin{array}{cc}
0 & 1 \\
1 & 0
\end{array}\right) \\
\sigma^{2}=-\bar{\sigma}^{2}=\left(\begin{array}{cc}
0 & -i \\
i & 0
\end{array}\right), & \sigma^{3}=-\bar{\sigma}^{3}=\left(\begin{array}{cc}
1 & 0 \\
0 & -1
\end{array}\right)
\end{array}
$$

and the indices are read, row by column, as $\left(\sigma^{\mu}\right)_{\alpha \dot{\alpha}}$ and $\left(\bar{\sigma}^{\mu}\right)^{\dot{\alpha} \alpha}$. For instance, $\left(\sigma^{2}\right)_{1 \dot{2}}=-i$.

The generators of the Lorentz transformations are

$$
\begin{aligned}
\sigma_{\alpha}^{\mu \nu \beta} & =\frac{1}{4}\left(\sigma_{\alpha \dot{\alpha}}^{\mu} \bar{\sigma}^{\nu \dot{\alpha} \beta}-\sigma_{\alpha \dot{\alpha}}^{\nu} \bar{\sigma}^{\mu \dot{\alpha} \beta}\right) \\
\bar{\sigma}_{\dot{\beta}}^{\mu \nu \dot{\alpha}} & =\frac{1}{4}\left(\bar{\sigma}^{\mu \dot{\alpha} \alpha} \sigma_{\alpha \dot{\beta}}^{\nu}-\bar{\sigma}^{\nu \dot{\alpha} \alpha} \sigma_{\alpha \dot{\beta}}^{\mu}\right) .
\end{aligned}
$$

Dotted and undotted indices describe two component Weyl spinors of opposite chirality and we raise them and lower them using the totally antisymmetric tensors $\epsilon_{21}=\epsilon^{12}=1$ and $\epsilon_{\dot{2} \dot{1}}=\epsilon^{i \dot{2}}=1$ :

$$
\psi^{\alpha}=\epsilon^{\alpha \beta} \psi_{\beta}, \quad \psi_{\alpha}=\epsilon_{\alpha \beta} \psi^{\beta}, \quad \bar{\psi}^{\dot{\alpha}}=\epsilon^{\dot{\alpha} \dot{\beta}} \bar{\psi}_{\dot{\beta}}, \quad \bar{\psi}_{\dot{\alpha}}=\epsilon_{\dot{\alpha} \dot{\beta}} \bar{\psi}^{\dot{\beta}} .
$$

Complex conjugation changes chirality: $\left(\psi_{\alpha}\right)^{*}=\bar{\psi}_{\dot{\alpha}}$ and when two pairs of indices are not explicitly written, the following summation conventions are understood:

$$
\psi \chi=\psi^{\alpha} \chi_{\alpha} \quad \text { and } \quad \bar{\psi} \bar{\chi}=\bar{\psi}_{\dot{\alpha}} \bar{\chi}^{\dot{\alpha}} .
$$

So far everything is as in [11] except for the signature of the metric. We now define the square of a spinor as

$$
\psi^{2}=\frac{1}{2} \psi^{\alpha} \psi_{\alpha} \quad \text { and } \quad \bar{\psi}^{2}=\frac{1}{2} \bar{\psi}_{\dot{\alpha}} \bar{\psi}^{\dot{\alpha}} .
$$


(This is done as to eliminate many of the powers of two appearing explicitly otherwise.)

Superspace has coordinates $x^{\mu}, \theta^{\alpha}, \bar{\theta}^{\dot{\alpha}}$ and the fermionic derivatives are defined as

$$
\partial_{\alpha}=\frac{\partial}{\partial \theta^{\alpha}} \quad \text { and } \quad \bar{\partial}_{\dot{\alpha}}=\frac{\partial}{\partial \bar{\theta}^{\dot{\alpha}}}
$$

so that $\partial_{\alpha} \theta^{\beta}=\delta_{\alpha}^{\beta}$ and $\bar{\partial}_{\dot{\alpha}} \bar{\theta}^{\dot{\beta}}=\delta_{\dot{\alpha}}^{\dot{\beta}}$.

The supercharges and superspace derivatives are defined as follows:

$$
\begin{aligned}
& Q_{\alpha}=\partial_{\alpha}-\frac{i}{2} \sigma_{\alpha \dot{\alpha}}^{\mu} \bar{\theta}^{\dot{\alpha}} \partial_{\mu} \quad \text { and } \quad \bar{Q}_{\dot{\alpha}}=\bar{\partial}_{\dot{\alpha}}-\frac{i}{2} \theta^{\alpha} \sigma_{\alpha \dot{\alpha}}^{\mu} \partial_{\mu} \\
& D_{\alpha}=\partial_{\alpha}+\frac{i}{2} \sigma_{\alpha \dot{\alpha}}^{\mu} \bar{\theta}^{\dot{\alpha}} \partial_{\mu} \quad \text { and } \quad \bar{D}_{\dot{\alpha}}=\bar{\partial}_{\dot{\alpha}}+\frac{i}{2} \theta^{\alpha} \sigma_{\alpha \dot{\alpha}}^{\mu} \partial_{\mu} \text {. }
\end{aligned}
$$

The only two non-vanishing anticommutators are

$$
\left\{Q_{\alpha}, \bar{Q}_{\dot{\beta}}\right\}=-\left\{D_{\alpha}, \bar{D}_{\dot{\beta}}\right\}=-i \sigma_{\alpha \dot{\beta}}^{\mu} \partial_{\mu}=-\sigma_{\alpha \dot{\beta}}^{\mu} P_{\mu}
$$

Indices on the derivatives $D_{\alpha}$ and $\bar{D}_{\dot{\beta}}$ are raised and lowered using the same conventions as in (218) but we define the square of the fermionic derivatives with an extra minus sign as compared to (220):

$$
D^{2}=-\frac{1}{2} D^{\alpha} D_{\alpha} \quad \text { and } \quad \bar{D}^{2}=-\frac{1}{2} \bar{D}_{\dot{\alpha}} \bar{D}^{\dot{\alpha}},
$$

so that: $D^{2} \theta^{2}=\bar{D}^{2} \bar{\theta}^{2}=+1$. The fermionic integrals are just another notation for the derivative: $\int d^{2} \theta \theta^{2}=\int d^{2} \bar{\theta} \bar{\theta}^{2}=1$. It is straightforward to check that these integrals are invariant under the translations $\theta \rightarrow \theta+\xi$ and $\bar{\theta} \rightarrow \bar{\theta}+\bar{\xi}$.

All the identities involving the $\sigma$ matrices described in the Appendices $\mathrm{A}$ and $\mathrm{B}$ of Wess and Bagger [11] are still valid by letting $\eta_{\mu \nu} \rightarrow-\eta_{\mu \nu}$ and recalling that $\theta \theta=2 \theta^{2}$ and $\bar{\theta} \bar{\theta}=2 \bar{\theta}^{2}$ in our notation.

\section{B One cut solution to the cubic matrix model}

In this appendix we show how to compute the leading (planar) contribution to the free energy $\mathcal{F}$ of the one cut cubic matrix model [10], thus completing the computation of Section 4. To be slightly more general, we present the computation for a generic potential $W$ and only at the end we substitute $W(z)=\frac{m}{2} z^{2}+\frac{g}{3} z^{3}$. 
We consider the following matrix integral for a generic potential $W(M)$ of a random hermitian matrix $M$ (here we write the trace explicitly)

$$
e^{-\frac{\hat{N}^{2}}{\hat{S}^{2}} \mathcal{F}+\ldots}=\int \mathrm{d}^{\hat{N}^{2}} M e^{-\frac{\hat{N}}{\hat{S}} \operatorname{tr} W(M)},
$$

where $\mathcal{F}$ is the planar free energy, the dots denote non-planar (sub-leading) contributions and with respect to Section 4 we have already substituted for $\epsilon=\hat{S} / \hat{N}$.

Since $M$ is hermitian, there exists a unitary matrix $\Omega$ such that

$$
M=\Omega^{\dagger} \Lambda \Omega,
$$

where $\Lambda=\operatorname{diag}\left(\lambda_{1}, \ldots, \lambda_{\hat{N}}\right)$ is a diagonal matrix with the real eigenvalues $\lambda_{i}$ of $M$ as entries. The measure in the new variables becomes $\mathrm{d}^{\hat{N}^{2}} M=$ $\mathrm{d}^{\hat{N}} \Lambda \mathrm{d}^{\hat{N}^{2}-\hat{N}} \Omega \Delta(\lambda)^{2}$ with $\Delta(\lambda)^{2}$ being the Jacobian of the transformation.

This Jacobian can be determined in the following way. The metric on the matrix space can be written as

$$
\begin{aligned}
\mathrm{d} s^{2} & =\operatorname{tr} \mathrm{d} M \mathrm{~d} M \\
& =\operatorname{tr}\left(\mathrm{d} \Omega^{\dagger} \Lambda \Omega+\Omega^{\dagger} \mathrm{d} \Lambda \Omega+\Omega^{\dagger} \Lambda \mathrm{d} \Omega\right)^{2} \\
& =\operatorname{tr}\left(\mathrm{d} \Lambda+\left[\Lambda, \mathrm{d} \Omega \Omega^{\dagger}\right]\right)^{2} \\
& =\operatorname{tr}\left(\mathrm{d} \Lambda^{2}+\left[\Lambda, \mathrm{d} \Omega \Omega^{\dagger}\right]^{2}\right) \\
& =\mathrm{d} \lambda_{1}^{2}+\ldots+\mathrm{d} \lambda_{\hat{N}}^{2}+\sum_{i \neq j}\left(\lambda_{i}-\lambda_{j}\right)^{2}\left|\mathrm{~d} \Omega_{i k} \Omega_{k j}^{\dagger}\right|^{2} .
\end{aligned}
$$

The Jacobian is just the square root of the determinant of this metric

$$
\Delta(\lambda)=\sqrt{\operatorname{det} G}=\prod_{i<j}\left(\lambda_{i}-\lambda_{j}\right)^{2} .
$$

This result can be inserted into eq. (225)

$$
\int \mathrm{d}^{\hat{N}^{2}} M e^{-\frac{\hat{N}}{S} \operatorname{tr} W(M)}=\int \mathrm{d}^{\hat{N}^{2}-\hat{N}} \Omega \mathrm{d}^{\hat{N}} \lambda \prod_{i<j}\left(\lambda_{i}-\lambda_{j}\right)^{2} e^{-\frac{\hat{N}}{S} \sum_{k} W\left(\lambda_{k}\right)} .
$$

The integral over the unitary matrices gives an overall constant that we absorb into the measure leaving

$$
\int \mathrm{d}^{\hat{N}^{2}} M e^{-\frac{\hat{N}}{S} \operatorname{tr} W(M)}=\int \mathrm{d}^{\hat{N}} \lambda e^{-\frac{\hat{N}}{S} \sum_{k} W\left(\lambda_{k}\right)+\sum_{i \neq j} \log \left|\lambda_{i}-\lambda_{j}\right|} .
$$


Solving this integral is impossible for finite $\hat{N}$. Instead, we use the steepest descent method for the evaluation, which corresponds to taking the large $\hat{N}$ limit, thus singling out the planar diagrams.

To leading order in $\hat{N}$, the free energy is given by

$$
-\frac{\hat{N}^{2}}{\hat{S}^{2}} \mathcal{F}=-\frac{\hat{N}}{\hat{S}} \sum_{k} W\left(\hat{\lambda}_{k}\right)+\sum_{i \neq j} \log \left|\hat{\lambda}_{i}-\hat{\lambda}_{j}\right|,
$$

where $\hat{\lambda}_{k}$ are the $\hat{N}$ solutions to the extremization problem given by the simultaneous solution of the following $\hat{N}$ equations, labeled by $k=1, \ldots \hat{N}$.

$$
0=-\frac{\hat{N}}{\hat{S}} W^{\prime}\left(\lambda_{k}\right)+2 \sum_{k \neq j} \frac{1}{\lambda_{k}-\lambda_{j}} .
$$

To solve this set of equations for large $\hat{N}$ one goes to a continuous description of the eigenvalues by defining

$$
\begin{aligned}
\frac{i}{\hat{N}} & \rightarrow x \in[0,1] \\
\sum_{i} & \rightarrow \hat{N} \int_{0}^{1} \mathrm{~d} x \\
\lambda_{i} & \rightarrow \lambda(x)
\end{aligned}
$$

With these identifications the free energy and the extremum equation become

$$
\begin{aligned}
\mathcal{F} & =\hat{S} \int_{0}^{1} \mathrm{~d} x W(\lambda(x))-\hat{S}^{2} \int_{0}^{1} \mathrm{~d} x \int_{0}^{1} \mathrm{~d} y \log |\lambda(x)-\lambda(y)| \\
0 & =-W^{\prime}(\lambda(x))+2 \hat{S} \int_{0}^{1} \mathrm{~d} y \frac{1}{\lambda(x)-\lambda(y)},
\end{aligned}
$$

where the integrals are to be thought of as principal values. Note that all dependence on $\hat{N}$ has disappeared and that we traded a set of $\hat{N}$ algebraic equations for one integral equation.

To proceed, introduce the density of eigenvalues $\rho(\lambda)$ as

$$
\hat{S} \mathrm{~d} x=\mathrm{d} \lambda \rho(\lambda)
$$

which is non-negative, has support within an interval $[a, b]$ (to be deter- 
mined $)^{28}$ and is normalized such that

$$
\int_{a}^{b} \mathrm{~d} \lambda \rho(\lambda)=\hat{S} .
$$

In terms of the eigenvalue density eqs. (234) and (235) read

$$
\begin{aligned}
\mathcal{F} & =\int_{a}^{b} \mathrm{~d} \lambda \rho(\lambda) W(\lambda)-\int_{a}^{b} \mathrm{~d} \lambda^{\prime} \int_{a}^{b} \mathrm{~d} \lambda \rho(\lambda) \rho\left(\lambda^{\prime}\right) \log \left|\lambda-\lambda^{\prime}\right| \\
0 & =-W^{\prime}(\lambda)+2 \int_{a}^{b} \mathrm{~d} \lambda^{\prime} \frac{\rho\left(\lambda^{\prime}\right)}{\lambda-\lambda^{\prime}} .
\end{aligned}
$$

Integrating (239) in the two intervals $[a, \lambda]$ and $[\lambda, b]$ and combining the results yields

$$
\begin{aligned}
& \int_{a}^{b} \mathrm{~d} \lambda^{\prime} \rho\left(\lambda^{\prime}\right) \log \left|\lambda-\lambda^{\prime}\right|=\frac{1}{4}(2 W(\lambda)-W(b)-W(a)) \\
& +\frac{1}{2} \int_{a}^{b} \mathrm{~d} \lambda^{\prime} \rho\left(\lambda^{\prime}\right) \log \left(b-\lambda^{\prime}\right)\left(\lambda^{\prime}-a\right)
\end{aligned}
$$

Eq. (238) then becomes, using the normalization of the eigenvalue density (237)

$$
\begin{aligned}
\mathcal{F} & =\frac{1}{2} \int_{a}^{b} \mathrm{~d} \lambda \rho(\lambda)(W(\lambda)-\hat{S} \log (b-\lambda)(\lambda-a)) \\
& +\frac{\hat{S}}{4}(W(b)+W(a)) .
\end{aligned}
$$

To find the eigenvalue density define the resolvent

$$
R(z)=\int_{-\infty}^{\infty} \mathrm{d} \lambda \frac{\rho(\lambda)}{z-\lambda}
$$

which is an analytic function of $z$. The singularity of the resolvent is a cut along the real interval $[a, b]$. Across the cut the resolvent has the following behavior

$$
\begin{aligned}
& R(\lambda+i \epsilon)+R(\lambda-i \epsilon)=2 \int_{a}^{b} \mathrm{~d} \lambda^{\prime} \frac{\rho\left(\lambda^{\prime}\right)}{\lambda-\lambda^{\prime}}=W^{\prime}(\lambda) \\
& R(\lambda+i \epsilon)-R(\lambda-i \epsilon)=\oint \mathrm{d} \lambda^{\prime} \frac{\rho\left(\lambda^{\prime}\right)}{\lambda-\lambda^{\prime}}=-2 \pi i \rho(\lambda) .
\end{aligned}
$$

\footnotetext{
${ }^{28}$ We focus here on the so called one cut solution, i.e. the one with all eigenvalues grouped around an extremum, corresponding to an unbroken gauge group.
} 
The integral in (243) is a principal value and the one in (244) is a residue as can be easily seen by drawing the contours. The idea now is to use (243) to find the resolvent and then (244) to extract the eigenvalue density $\rho$.

One particular solution to eq. (243) is, of course, the regular solution $R_{\text {reg }}(z)=\frac{1}{2} W^{\prime}(z)$, but this cannot be the full story because it does not have the right asymptotic behavior at infinity. To $R_{r e g}(z)$ we must add a generic solution to the homogeneous equation

$$
R_{\text {sing }}(\lambda+i \epsilon)+R_{\text {sing }}(\lambda-i \epsilon)=0,
$$

necessarily singular along the cut and chosen so that the full solution has the correct large $z$ behavior following from (242):

$$
R(z)=R_{\text {sing }}(z)+R_{\text {reg }}(z) \sim \frac{\hat{S}}{z}+\mathcal{O}\left(\frac{1}{z^{2}}\right), \quad z \rightarrow \infty .
$$

Across the cut we need a sign change which is implemented by

$$
R_{\text {sing }}(z)=P(z) \sqrt{(z-a)(z-b)},
$$

where $P(z)$ is a regular polynomial with degree two less than the degree of $W(z)$ in order to cancel the various powers of $z$. The coefficients in $P(z)$ are given implicitly by the condition (246).

The sought eigenvalue density $\rho(\lambda)$ is then, using eq. (244)

$$
\rho(\lambda)=\frac{1}{\pi i} R_{\text {sing }}(\lambda)
$$

where the sign of the square root is chosen to give $\rho$ non negative. Having determined the eigenvalue density, the free energy can be calculated by evaluating (241).

Up to this stage the potential $W(M)$ is generic. To illustrate the technique and to give the full solution to the problem of Section 4, we now specialize to a cubic matrix model. Notice that although the integral with a cubic potential is not convergent, each term in the perturbative expansion is, and this is all we need. Let us thus take, as in Section 4

$$
W(M)=\frac{m}{2} M^{2}+\frac{g}{3} M^{3} .
$$

Requiring that there are no $\mathcal{O}\left(z^{2}\right)$ and $\mathcal{O}(z)$ terms in $R(z)$ in the large $z$ limit, we get:

$$
P(z)=-\frac{1}{4}(2 m+g(a+b)+2 g z) .
$$


Furthermore, by canceling the constant term $\mathcal{O}\left(z^{0}\right)$ and requiring the pole to have residue $\hat{S}$, we obtain conditions on the end points $a$ and $b$ of the interval where the eigenvalue density has support

$$
\begin{aligned}
0 & =2 g(a+b)^{2}+g(a-b)^{2}+4 m(a+b) \\
16 \hat{S} & =(a-b)^{2}(g(a+b)+m) .
\end{aligned}
$$

Thus, from eq. (244):

$$
\rho(\lambda)=\frac{1}{4 \pi}(2 g \lambda+2 m+g(a+b)) \sqrt{(b-\lambda)(\lambda-a)} .
$$

The free energy is computed by using the eigenvalue density and evaluating eq. (241)

$$
\begin{aligned}
\mathcal{F}= & \frac{1}{8 \pi} \int_{a}^{b} \mathrm{~d} \lambda[(2 g \lambda+2 m+g(a+b)) \sqrt{(b-\lambda)(\lambda-a)} \\
& \left.\left(\frac{m}{2} \lambda^{2}+\frac{g}{3} \lambda^{3}-\hat{S} \log |(b-\lambda)(\lambda-a)|\right)\right] \\
+ & \frac{\hat{S}}{4}\left(\frac{m}{2}\left(a^{2}+b^{2}\right)+\frac{g}{3}\left(a^{3}+b^{3}\right)\right)
\end{aligned}
$$

It is convenient to introduce a parameter

$$
\sigma \equiv \frac{g}{2 m}(a+b)
$$

Then the constraints (251) and (252) imply

$$
2 \frac{g^{2}}{m^{3}} \hat{S}+\sigma(1+\sigma)(1+2 \sigma)=0
$$

where we chose the branch $\sigma=0$ for $g=0$. Evaluating the integral (254) keeping only terms that depend on $g$ (through $\sigma$ ) yields ${ }^{29}$ :

$$
\mathcal{F}=-\frac{\hat{S}^{2}}{3} \frac{\sigma\left(2+6 \sigma+3 \sigma^{2}\right)}{(1+\sigma)(1+2 \sigma)^{2}}+\frac{\hat{S}^{2}}{2} \log (1+2 \sigma) .
$$

The constraint (256) can be solved in a perturbation expansion in the coupling constant $g$ and substituting into $\mathcal{F}$ we get:

$$
\mathcal{F}=-\frac{2 g^{2} \hat{S}^{3}}{3 m^{3}}-\frac{8 g^{4} \hat{S}^{4}}{3 m^{6}}-\frac{56 g^{6} \hat{S}^{5}}{3 m^{9}}-\frac{512 g^{8} \hat{S}^{6}}{3 m^{12}}-\frac{9152 g^{10} \hat{S}^{7}}{5 m^{15}}+\mathcal{O}\left(g^{12}\right)
$$

The superpotential for the gauge theory is then given by the DijkgraafVafa formula (124) of Section 4.

\footnotetext{
${ }^{29} \mathrm{~A}$ constant term arising at $g=0$ can always be absorbed into the measure as we have done many times before.
} 


\section{Bibliographical note}

As mentioned in the Introduction, the work of Dijkgraaf and Vafa [1 has generated many other interesting developments that unfortunately did not find their place in our review. In this Appendix we make an attempt to summarize them hoping that this will help the reader in finding his/her own way through the literature. We apologize for any omission or imprecision that might occur.

The most important omission is, of course, a discussion of the geometrical aspects and implications of the work of Dijkgraaf and Vafa. This is particularly embarrassing because this was the original route through which the equivalence with the matrix model was originally discovered! This subject deserves its own review written by someone more qualified than us to do it justice - here we only refer the interested reader to some of the related literature [38, that followed [1].

Within the realm of gauge theories, probably the most important omission is the discussion of theories with extended SUSY. The work of [1] has a natural extension to theories with $\mathcal{N}=2$ and $\mathcal{N}=4$ SUSY. In the $\mathcal{N}=2$ context [39] many of the results of Seiberg-Witten theory [6] have been reproduced using matrix models. Various extensions to gauge groups other than $U(N)$ and the addition of flavor hypermultiplets have also been discussed. In the $\mathcal{N}=4$ case [40], much attention has been given to extracting S-duality and to the various deformations allowed by the presence of three chiral multiplets in the adjoint representation. In particular, one can study either the Leigh-Strassler deformation [41] or the relevant deformations caused by adding mass terms to some of the chiral multiplets leading to the so called $\mathcal{N}=1^{*}$ or $\mathcal{N}=2^{*}$ theories 42 .

The new techniques to study SUSY gauge theories immediately call for their application to the study of various non-perturbative aspects of their dynamics. We greatly regret not having discussed the new results emerging from these investigations such as a much better understanding of the parameter space of these theories [43], (see also [44]), the analysis [45] of the Argyres-Douglas points [46] and that [47] of Seiberg duality [8].

Another notable omission is the discussion of gravitational corrections 49. In the presence of a non-trivial background for the gravitino, the analysis that led to the vanishing of non-planar diagrams is no longer valid because the gravitino can soak up additional zero modes. The non-planar

contributions have also been checked to agree with previously known results with both a diagrammatic analysis and a computation based on the anomaly. This has also led to an interesting deformation of the chiral ring where 
"Grassmann" operators are no longer nilpotent even at the classical level.

Returning to gauge theory, the matrix model has been applied to the study of theories based on other gauge groups such as $S O(N)$ and $S p(N)$ without [50] or with [51] flavors as well as $U(N)$ with symmetric or antisymmetric representations. (The case of $U(N)$ with "flavor" matter in the fundamental has already briefly been discussed in the main text [37, see also [52.) The case of $S U(N)$ with a number of flavors equal to the number of colors is particularly interesting because it allows for baryonic deformations [53]. Various aspects of this model have been investigated in [54].

Moving on to more "exotic" cases, orbifolds and quivers yielding semisimple groups have been studied in [55, whereas multi-trace deformations have been discussed in [56].

Regrettably, the study of chiral gauge theories using these new techniques has not received much attention. These theories are interesting because they can [32] and they do [57] break SUSY dynamically. Some examples are discussed in [36, see also [58.

Still within the realm of applications to four dimensional gauge theories, a justification of the linearity principle of [20] from the matrix model has been proposed in [59] and an attempt at deriving the VY term from a diagrammatic expansion has been made in 60. A thorough study of some of the more formal aspects of the matrix model has been done in the series of papers 61. (See 62 for a discussion of the gauged model and the role played by the ghosts and 63] for some studies on supersymmetric matrix models). Finally, although the original results are strictly four dimensional as should be clear from the discussion of Section 4, extensions of some results to both lower 64] and higher 65] dimensions have also appeared.

\section{References}

[1] R. Dijkgraaf and C. Vafa, arXiv:hep-th/0208048

[2] D. Amati, K. Konishi, Y. Meurice, G. C. Rossi and G. Veneziano, Phys. Rept. 162 (1988) 169.

[3] K. A. Intriligator and N. Seiberg, Nucl. Phys. Proc. Suppl. 45BC (1996) 1 arXiv:hep-th/9509066.

M. A. Shifman, Prog. Part. Nucl. Phys. 39 (1997) 1 arXiv:hep-th/9704114.

D. S. Berman and E. Rabinovici, arXiv:hep-th/0210044. 
[4] R. Gopakumar and C. Vafa, Adv. Theor. Math. Phys. 3 (1999) 1415 arXiv:hep-th/9811131.

C. Vafa, J. Math. Phys. 42 (2001) 2798 arXiv:hep-th/0008142.

F. Cachazo, K. A. Intriligator and C. Vafa, Nucl. Phys. B 603 (2001) 3 arXiv:hep-th/0103067.

R. Dijkgraaf and C. Vafa, Nucl. Phys. B 644 (2002) 3 arXiv:hep-th/0206255.

R. Dijkgraaf and C. Vafa, Nucl. Phys. B 644 (2002) 21 arXiv:hep-th/0207106.

[5] F. Ferrari, Nucl. Phys. B 648 (2003) 161 arXiv:hep-th/0210135.

[6] N. Seiberg and E. Witten, Nucl. Phys. B 426 (1994) 19 [Erratum-ibid. B 430 (1994) 485] arXiv:hep-th/9407087.

N. Seiberg and E. Witten, Nucl. Phys. B 431 (1994) 484 arXiv:hep-th/9408099.

[7] R. Dijkgraaf, M. T. Grisaru, C. S. Lam, C. Vafa and D. Zanon, arXiv:hep-th/0211017

[8] F. Cachazo, M. R. Douglas, N. Seiberg and E. Witten, JHEP 0212 (2002) 071 arXiv:hep-th/0211170.

[9] K. Konishi, Phys. Lett. B 135 (1984) 439.

K. Konishi and K. Shizuya, Nuovo Cim. A 90 (1985) 111.

[10] E. Brezin, C. Itzykson, G. Parisi and J. B. Zuber, Commun. Math. Phys. 59 (1978) 35.

[11] J. Wess and J. Bagger Supersymmetry and Supergravity, Princeton Series in Physics (1992)

[12] J. Wess and B. Zumino, Phys. Lett. B 49 (1974) 52.

[13] S. Ferrara and B. Zumino, Nucl. Phys. B 79 (1974) 413.

A. Salam and J. Strathdee, Phys. Lett. B 51 (1974) 353.

[14] A. Salam and J. Strathdee, Nucl. Phys. B 76 (1974) 477.

[15] M. A. Luty and W. I. Taylor, Phys. Rev. D 53 (1996) 3399 arXiv:hep-th/9506098. 
[16] M. T. Grisaru, W. Siegel and M. Rocek, Nucl. Phys. B 159 (1979) 429.

[17] P. C. West, Phys. Lett. B 258 (1991) 375.

I. Jack, D. R. Jones and P. C. West, Phys. Lett. B 258 (1991) 382.

[18] N. Seiberg, Phys. Lett. B 318 (1993) 469 arXiv:hep-ph/9309335.

[19] M. A. Shifman and A. I. Vainshtein, Nucl. Phys. B 359 (1991) 571.

[20] K. A. Intriligator, R. G. Leigh and N. Seiberg, Phys. Rev. D 50 (1994) 1092 arXiv:hep-th/9403198.

K. A. Intriligator, Phys. Lett. B 336 (1994) 409 arXiv:hep-th/9407106.

[21] I. Affleck, M. Dine and N. Seiberg, Nucl. Phys. B 241 (1984) 493.

I. Affleck, M. Dine and N. Seiberg, Nucl. Phys. B 256 (1985) 557.

[22] G. Veneziano and S. Yankielowicz, Phys. Lett. B 113 (1982) 231.

T. R. Taylor, G. Veneziano and S. Yankielowicz, Nucl. Phys. B 218 (1983) 493.

[23] N. Dorey, T. J. Hollowood, V. V. Khoze and M. P. Mattis, Phys. Rept. 371 (2002) 231 arXiv:hep-th/0206063.

[24] D. Finnell and P. Pouliot, Nucl. Phys. B 453 (1995) 225 arXiv:hep-th/9503115.

[25] P. Kraus and M. Shigemori, JHEP $0304 \quad$ (2003) 052 arXiv:hep-th/0303104.

L. F. Alday and M. Cirafici, JHEP $0305 \quad$ (2003) 041 arXiv:hep-th/0304119.

P. Kraus, A. V. Ryzhov and M. Shigemori, JHEP 0305 (2003) 059 arXiv:hep-th/0304138.

M. Aganagic, K. Intriligator, C. Vafa and N. P. Warner, arXiv:hep-th/0304271.

F. Cachazo, arXiv:hep-th/0307063

M. Matone, JHEP 0310 (2003) 068 arXiv:hep-th/0307285.

K. Landsteiner and C. I. Lazaroiu, arXiv:hep-th/0310111.

[26] G. 't Hooft, Nucl. Phys. B 72 (1974) 461. 
[27] H. Ooguri and C. Vafa, Nucl. Phys. B 641 (2002) 3 arXiv:hep-th/0205297.

[28] G. Hailu and H. Georgi, arXiv:hep-th/0401101.

[29] V. A. Novikov, M. A. Shifman, A. I. Vainshtein and V. I. Zakharov, Nucl. Phys. B 229 (1983) 407.

[30] E. Witten, arXiv:hep-th/0302194.

[31] P. Etingof and V. Kac, arXiv:math.qa/0305175

[32] E. Witten, Nucl. Phys. B 202 (1982) 253.

[33] A. Ceresole, G. Dall'Agata, R. D'Auria and S. Ferrara, Phys. Rev. D 61 (2000) 066001 arXiv:hep-th/9905226.

[34] N. Seiberg, JHEP 0301 (2003) 061 arXiv:hep-th/0212225.

[35] A. Gorsky, Phys. Lett. B 554 (2003) 185 arXiv:hep-th/0210281.

[36] A. Brandhuber, H. Ita, H. Nieder, Y. Oz and C. Romelsberger, arXiv:hep-th/0303001.

[37] R. Argurio, V. L. Campos, G. Ferretti and R. Heise, Phys. Rev. D 67 (2003) 065005 arXiv:hep-th/0210291.

[38] M. Aganagic, A. Klemm, M. Marino and C. Vafa, arXiv:hep-th/0211098

B. Feng, Nucl. Phys. B 661 (2003) 113 arXiv:hep-th/0212010.

R. Roiban, R. Tatar and J. Walcher, Nucl. Phys. B 665 (2003) 211 arXiv:hep-th/0301217.

C. I. Lazaroiu, JHEP 0305 (2003) 044 arXiv:hep-th/0303008.

A. Klemm, K. Landsteiner, C. I. Lazaroiu and I. Runkel, JHEP 0305 (2003) 066 arXiv:hep-th/0303032.

D. Berenstein, JHEP 0306 (2003) 019 arXiv:hep-th/0303033.

N. Halmagyi and V. Yasnov, arXiv:hep-th/0305134.

K. Landsteiner, C. I. Lazaroiu and R. Tatar, arXiv:hep-th/0306236.

F. Ferrari, arXiv:hep-th/0309151 
[39] S. G. Naculich, H. J. Schnitzer and N. Wyllard, Nucl. Phys. B 651 (2003) 106 arXiv:hep-th/0211123.

H. Itoyama and A. Morozov, Nucl. Phys. B 657 (2003) 53 arXiv:hep-th/0211245.

S. G. Naculich, H. J. Schnitzer and N. Wyllard, JHEP 0301 (2003) 015 arXiv:hep-th/0211254.

Y. Demasure and R. A. Janik, Nucl. Phys. B 661 (2003) 153 arXiv:hep-th/0212212.

M. Matone, Nucl. Phys. B 656 (2003) 78 arXiv:hep-th/0212253.

C. h. Ahn and S. k. Nam, Phys. Rev. D 67 (2003) 105022 arXiv:hep-th/0301203.

R. Abbaspur, A. Imaanpur and S. Parvizi, JHEP 0307 (2003) 043 arXiv:hep-th/0302083.

S. G. Naculich, H. J. Schnitzer and N. Wyllard, arXiv:hep-th/0305263

[40] N. Dorey, T. J. Hollowood, S. Prem Kumar and A. Sinkovics, JHEP 0211 (2002) 039 arXiv:hep-th/0209089.

N. Dorey, T. J. Hollowood, S. P. Kumar and A. Sinkovics, JHEP 0211 (2002) 040 arXiv:hep-th/0209099.

N. Dorey, T. J. Hollowood and S. P. Kumar, JHEP 0212 (2002) 003 arXiv:hep-th/0210239.

T. J. Hollowood, JHEP 0304 (2003) 025 arXiv:hep-th/0212065.

T. Mansson, JHEP 0303 (2003) 055 arXiv:hep-th/0302077.

M. Petrini, A. Tomasiello and A. Zaffaroni, JHEP 0308 (2003) 004 arXiv:hep-th/0304251.

T. Itoh, JHEP 0307 (2003) 005 arXiv:hep-th/0306035.

[41] R. G. Leigh and M. J. Strassler, Nucl. Phys. B 447 (1995) 95 arXiv:hep-th/9503121.

[42] J. Polchinski and M. J. Strassler, arXiv:hep-th/0003136.

[43] F. Ferrari, Phys. Rev. D 67 (2003) 085013 arXiv:hep-th/0211069.

F. Cachazo, N. Seiberg and E. Witten, JHEP 0302 (2003) 042 arXiv:hep-th/0301006.

F. Ferrari, Phys. Lett. B 557 (2003) 290 arXiv:hep-th/0301157. 
F. Cachazo, N. Seiberg and E. Witten, JHEP 0304 (2003) 018 arXiv:hep-th/0303207.

[44] T. Friedmann, Nucl. Phys. B 635 (2002) 384 arXiv:hep-th/0203256.

D. Berenstein, Phys. Lett. B 552 (2003) 255 arXiv:hep-th/0210183.

R. Gopakumar, JHEP 0305 (2003) 033 arXiv:hep-th/0211100.

K. Ohta, JHEP 0302 (2003) 057 arXiv:hep-th/0212025.

C. h. Ahn and Y. Ookouchi, JHEP $0303 \quad$ (2003) 010 arXiv:hep-th/0302150.

V. Balasubramanian, B. Feng, M. x. Huang and A. Naqvi, arXiv:hep-th/0303065

T. J. Hollowood, arXiv:hep-th/0305023

M. Matone and L. Mazzucato, JHEP $0307 \quad$ (2003) 015 arXiv:hep-th/0305225.

M. Matone and L. Mazzucato, arXiv:hep-th/0307130.

P. Svrcek, arXiv:hep-th/0308037

M. Klein and S. J. Sin, arXiv:hep-th/0309044.

H. Fuji and S. Mizoguchi, arXiv:hep-th/0309049.

C. Ahn and Y. Ookouchi, arXiv:hep-th/0309156.

M. Klein and S. J. Sin, arXiv:hep-th/0310078

[45] T. Eguchi and Y. Sugawara, JHEP $0305 \quad$ (2003) 063 arXiv:hep-th/0305050.

G. Bertoldi, JHEP 0306 (2003) 027 arXiv:hep-th/0305058.

D. Shih, arXiv:hep-th/0308001.

M. A. Ganjali, arXiv:hep-th/0309097.

[46] P. C. Argyres and M. R. Douglas, Nucl. Phys. B 448 (1995) 93 arXiv:hep-th/9505062.

[47] B. Feng and Y. H. He, Phys. Lett. B $562 \quad$ (2003) 339 arXiv:hep-th/0211234.

B. Feng, arXiv:hep-th/0211202.

B. Feng, Phys. Lett. B 572 (2003) 68 arXiv:hep-th/0303144.

[48] N. Seiberg, Nucl. Phys. B 435 (1995) 129 arXiv:hep-th/9411149. 
[49] A. Klemm, M. Marino and S. Theisen, JHEP 0303 (2003) 051 arXiv:hep-th/0211216.

R. Dijkgraaf, A. Sinkovics and M. Temurhan, arXiv:hep-th/0211241.

H. Ooguri and C. Vafa, arXiv:hep-th/0302109.

H. Ooguri and C. Vafa, arXiv:hep-th/0303063

J. R. David, E. Gava and K. S. Narain, arXiv:hep-th/0304227.

L. F. Alday, M. Cirafici, J. R. David, E. Gava and K. S. Narain, arXiv:hep-th/0305217.

L. F. Alday and M. Cirafici, JHEP 0309 (2003) 031 arXiv:hep-th/0306299.

F. Ardalan and N. Sadooghi, arXiv:hep-th/0307155.

H. Ita, H. Nieder and Y. Oz, arXiv:hep-th/0309041.

R. Dijkgraaf, M. T. Grisaru, H. Ooguri, C. Vafa and D. Zanon, arXiv:hep-th/0310061.

[50] H. Fuji and Y. Ookouchi, JHEP 0212 (2002) 067 arXiv:hep-th/0210148.

H. Ita, H. Nieder and Y. Oz, JHEP $0301 \quad$ (2003) 018 arXiv:hep-th/0211261.

S. K. Ashok, R. Corrado, N. Halmagyi, K. D. Kennaway and C. Romelsberger, Phys. Rev. D 67 (2003) 086004 arXiv:hep-th/0211291.

R. A. Janik and N. A. Obers, Phys. Lett. B 553 (2003) 309 arXiv:hep-th/0212069.

C. h. Ahn and S. Nam, Phys. Lett. B $562 \quad$ (2003) 141 arXiv:hep-th/0212231.

[51] Y. Ookouchi, arXiv:hep-th/0211287.

C. h. Ahn, Phys. Lett. B 560 (2003) 116 arXiv:hep-th/0301011.

Y. Ookouchi and Y. Watabiki, Mod. Phys. Lett. A 18 (2003) 1113 arXiv:hep-th/0301226.

C. h. Ahn, B. Feng and Y. Ookouchi, arXiv:hep-th/0306068.

C. h. Ahn, B. Feng and Y. Ookouchi, arXiv:hep-th/0307190.

[52] J. McGreevy, JHEP 0301 (2003) 047 arXiv:hep-th/0211009.

H. Suzuki, JHEP 0303 (2003) 005 arXiv:hep-th/0211052. 
I. Bena and R. Roiban, Phys. Lett. B 555 (2003) 117 arXiv:hep-th/0211075.

Y. Demasure and R. A. Janik, Phys. Lett. B 553 (2003) 105 arXiv:hep-th/0211082.

Y. Tachikawa, arXiv:hep-th/0211189.

I. Bena, S. de Haro and R. Roiban, Nucl. Phys. B 664 (2003) 45 arXiv:hep-th/0212083.

C. Hofman, arXiv:hep-th/0212095.

B. Feng, Phys. Rev. D 68 (2003) 025010 arXiv:hep-th/0212274.

Y. Nakayama, JHEP 0308 (2003) 049 arXiv:hep-th/0306007.

Y. Demasure, arXiv:hep-th/0307082.

P. Merlatti, arXiv:hep-th/0307115

B. M. Gripaios and J. F. Wheater, arXiv:hep-th/0307176.

R. A. Janik, arXiv:hep-th/0309084.

B. M. Gripaios, arXiv:hep-th/0311025

[53] N. Seiberg, Phys. Rev. D 49 (1994) 6857 arXiv:hep-th/9402044.

[54] R. Argurio, V. L. Campos, G. Ferretti and R. Heise, Phys. Lett. B 553 (2003) 332 arXiv:hep-th/0211249.

I. Bena, R. Roiban and R. Tatar, arXiv:hep-th/0211271.

H. Suzuki, JHEP 0303 (2003) 036 arXiv:hep-th/0212121.

I. Bena, H. Murayama, R. Roiban and R. Tatar, JHEP 0305 (2003) 049 arXiv:hep-th/0303115.

S. Corley, arXiv:hep-th/0305096.

[55] T. J. Hollowood and T. Kingaby, JHEP 0301 (2003) 005 arXiv:hep-th/0210096.

R. Dijkgraaf, A. Neitzke and C. Vafa, arXiv:hep-th/0211194,

S. Seki, Nucl. Phys. B 661 (2003) 257 arXiv:hep-th/0212079.

S. G. Naculich, H. J. Schnitzer and N. Wyllard, JHEP 0308 (2003) 021 arXiv:hep-th/0303268.

R. Casero and E. Trincherini, arXiv:hep-th/0304123.

R. Casero and E. Trincherini, arXiv:hep-th/0307054. 
[56] V. Balasubramanian, J. de Boer, B. Feng, Y. H. He, M. x. Huang, V. Jejjala and A. Naqvi, arXiv:hep-th/0212082.

M. Alishahiha and H. Yavartanoo, arXiv:hep-th/0303074.

[57] I. Affleck, M. Dine and N. Seiberg, Phys. Lett. B 137 (1984) 187.

I. Affleck, M. Dine and N. Seiberg, Phys. Rev. Lett. 52 (1984) 1677.

[58] R. Argurio, G. Ferretti and R. Heise, JHEP 0307 (2003) 044 arXiv:hep-th/0306125.

K. Landsteiner, C. I. Lazaroiu and R. Tatar, arXiv:hep-th/0307182.

K. Landsteiner, C. I. Lazaroiu and R. Tatar, arXiv:hep-th/0310052.

[59] Y. Tachikawa, arXiv:hep-th/0211274

[60] J. Ambjorn and R. A. Janik, Phys. Lett. B 569 (2003) 81 arXiv:hep-th/0306242.

[61] L. Chekhov and A. Mironov, Phys. Lett. B 552 (2003) 293 arXiv:hep-th/0209085.

V. A. Kazakov and A. Marshakov, J. Phys. A 36 (2003) 3107 arXiv:hep-th/0211236.

H. Itoyama and A. Morozov, Phys. Lett. B 555 (2003) 287 arXiv:hep-th/0211259.

H. Itoyama and A. Morozov, Prog. Theor. Phys. 109 (2003) 433 arXiv:hep-th/0212032.

L. Chekhov, A. Marshakov, A. Mironov and D. Vasiliev, Phys. Lett. B 562 (2003) 323 arXiv:hep-th/0301071.

A. Dymarsky and V. Pestun, Phys. Rev. D 67 (2003) 125001 arXiv:hep-th/0301135.

H. Itoyama and A. Morozov, arXiv:hep-th/0301136

A. Mironov, Fortsch. Phys. 51 (2003) 781 arXiv:hep-th/0301196.

S. Aoyama and T. Masuda, arXiv:hep-th/0309232

A. Alexandrov, A. Mironov and A. Morozov, arXiv:hep-th/0310113.

[62] R. Dijkgraaf, S. Gukov, V. A. Kazakov and C. Vafa, Phys. Rev. D 68 (2003) 045007 arXiv:hep-th/0210238. 
[63] H. Kawai, T. Kuroki and T. Morita, Nucl. Phys. B 664 (2003) 185 arXiv:hep-th/0303210.

H. Itoyama and H. Kanno, arXiv:hep-th/0304184.

[64] R. Boels, J. de Boer, R. Duivenvoorden and J. Wijnhout, arXiv:hep-th/0304061.

M. Alishahiha and A. E. Mosaffa, JHEP 0305 (2003) 064 arXiv:hep-th/0304247.

R. Boels, J. de Boer, R. Duivenvoorden and J. Wijnhout, arXiv:hep-th/0305189.

M. Alishahiha, J. de Boer, A. E. Mosaffa and J. Wijnhout, arXiv:hep-th/0308120.

A. Ritz, arXiv:hep-th/0308144.

[65] R. Dijkgraaf and C. Vafa, arXiv:hep-th/0302011

T. J. Hollowood, JHEP 0303 (2003) 039 arXiv:hep-th/0302165.

I. Bena and R. Roiban, arXiv:hep-th/0308013.

T. J. Hollowood, A. Iqbal and C. Vafa, arXiv:hep-th/0310272

S. Ganguli, O. J. Ganor and J. A. Gill, arXiv:hep-th/0311042. 